九州大学学術情報リポジトリ

Kyushu University Institutional Repository

Petrochemical Study of Basic and ULtrabasic Inclusions in Basaltic Rocks from Northern Kyushu, Japan

Ishibashi, Kiyoshi

Faculty of Science, Kyushu University

https://doi.org/10.5109/1543910

出版情報：九州大學理學部紀要：Series D, Geology. 20（1)，pp.85-146，1970-01-25. Faculty of Science, Kyushu University バージョン：

権利関係 : 
Mem. Fac. Sci., Kyushu Univ., Ser. D, Geology, Vol. XX, No. 1, pp. $85-146$, text-figs. $1-26$, tables. $1-21$, pls. $18-25$, Jan. 25,1970

\title{
Petrochemical Study of Basic and Ultrabasic Inclusions in Basaltic Rocks from Northern Kyushu, Japan
}

\author{
By
}

\author{
Kiyoshi IsHIBASHI
}

\begin{abstract}
Basic and ultrabasic inclusions are found in the post-Miocene alkali olivine basalts of the southwestern Japan. A sharp boundary of the most inclusions with basaltic host rocks and translation lamellae of some constituent minerals suggest that the inclusions were captured in the solid state by basaltic magmas. The main constituent minerals of the inclusions are olivine, orthopyroxene, clinopyroxene, chromite-spinel and plagioclase. All of them are separated from the host rocks and investigated through chemical and X-ray analyses.

New chemical data of nine spinels, ten olivines, twenty-five clinopyroxenes, ten orthopyroxenes and five plagioclases are given. Bulk chemical composition of basaltic and limburgitic host rocks and also chemical composition of five inclusions are presented.

On the grounds of the mode of occurrence, petrological and chemical characters of constituent minerals, as well as geotectonic considerations, the basic and ultrabasic inclusions from southwestern Japan can be divided into two groups on account of the assemblage of silicate and oxide minerals as follows:

(1) The green type inclusions, i.e. the group of dunite, Cr-diopside peridotite, Cr-pyroxenite and anorthite-bearing Cr-pyroxenite.

(2) The black type inclusions, i.e. the group of Al-pyroxenite, olivine gabbro and olivine-free gabbro.

The green type inclusions are probably derived from the upper mantle materials brought up with basaltic magma, whereas the black type inclusions are formed on one hand by the reaction of the green type inclusions with the enclosing basaltic magma, and on the other hand, a part of them is formed by settling of early formed crystals in the basaltic magma.

The zonal distribution of the basic and ultrabasic inclusions and acid xenolithes in the post Miocene basaltic rocks is discussed briefly on the basis of geotectonic model of the Japanese Islands.
\end{abstract}

\section{Contents}

I. Introduction

II. Geological setting and petrography of basic and ultrabasic inclusions in basaltic rocks

A. Basic and ultrabasic inclusions from Takashima island
a) Dunite
b) Cr-diopside peridotite
c) Cr-pyroxenite and anorthite-bearing Cr-pyroxenite
d) Al-pyroxenite

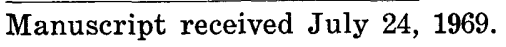


B. Gabbro and dunite in the basaltic rocks from Kurose in the Hakata Bay, Fukuoka City

a) Olivine gabbro and olivine-free gabbro

b) Dunite

C. Peridotite inclusions in the limburgite from Ogusoyama, Shimane Prefecture

III. Modal compositions and optical properties of constituent minerals of basic and ultrabasic inclusions

IV. Chemical and X-ray analyses

A. Preparation of sample for chemical and X-ray studies

B. Method of chemical analysis of silicate minerals

C. Method of chemical analysis of spinel and chromite

D. List of analyzed samples

E. Chemical composition of constituent minerals

a) Spinel group

1) Chemical composition of spinel group

2) X-ray analyses of spinel group

b) Olivine

1) Chemical composition of olivine

2) $\mathrm{NiO}$ content in olivine

c) Clinopyroxene

1) Chemical composition of clinopyroxene

2) The relation between $2-\mathrm{Si}$ and $\mathrm{Al} / \mathrm{Cr}$ in clinopyroxene

d) Orthopyroxene

1) Chemical composition of orthopyroxene

2) The tie line relation of co-existing pyroxene

3) The tie line relation of olivine and clinopyroxene and of olivine and orthopyroxene

e) Plagioclase

1) Chemical composition of plagioclase

E. Bulk chemical composition of basic and ultrabasic inclusions in basaltic rocks

V. Discussion

A. Two distinct groups of inclusions in the basaltic rocks from the region of northern Kyushu and San-in

B. Relation between olivine-rich inclusions and basaltic host rocks

C. The zonal distribution of basic and ultrabasic inclusions in the Cenozoic basaltic rocks of southwestern Japan

VI. Summary of results

VII. References

Plates

\section{Introduction}

The basaltic magmas may be produced in the upper part of the mantle by sudden release of pressure (YODER and TILLEY, 1964) or by the elevation of temperature which exceeds over the melting point of the mantle materials. The magma, however, does not always exist in the mantle but it occurs in definite time and in definite scale. In recent years the basic and ultrabasic inclusions in the basaltic rocks have become more and more important to study the generation of basaltic magmas. The reason is essentially due to the assumption that basaltic magmas are produced by the partial fusion of the mantle materials, and basic and ultrabasic inclusions in basaltic rocks are unmelted part of the mantle materials or residue of the mantle matter brought up with basaltic magmas. 
It is, however, impossible to see the mantle at present. For this reason, the great efforts have been made by geologists, geochemists, petrologists and geophysicists to find out the information about the mantle by various methods of investigations of the basic and ultrabasic inclusions in basaltic rocks.

Many authors have discussed the origin of olivine-rich inclusions in basaltic rocks in connection with host rocks. Essentially the discussions can be divided broadly into two groups; one is the hypothesis of cognate origin and the other is that of accidental one. They are still in controversy. The former hypothesis is based on the fabric, mineralogical composition and mode of occurrences of the inclusions, as has been maintained by LACROIX (1932), TURNER and VERHOOGEN (1951), BRown (1955) and SEARLE (1960).

They considered that the olivine-rich inclusions are formed by the accumulation of crystals in the early stage from the enclosing basaltic magma and/or related magmas. Olivine-rich inclusions are, however, usually found in alkali basalts and/or basic volcanic rocks with alkaline affinity and almost absent in the tholeiitic basalts (KUNO, 1960; WHITE, 1966).

Ross et al. (1954), DERIU (1959) and WILSHIRE and BINNS (1961) have pointed out that, in spite of the great variability in the chemical composition of basaltic host rocks, the olivine-rich inclusions in them show a remarkable uniform composition of constituent minerals. The distribution of the inclusions is world-wide scale. This argument has no agreement with the hypothesis of crystal accumulation.

The latter hypothesis is based on the similarity of the chemical composition of constituent minerals, the texture and the shape of world-wide distribution of the inclusions. (Ross et al., 1954).

WILSHIRE and BINNS (1961) have suggested that the inclusions are the fragment of unmelted mantle materials brought up with basaltic magmas.

The relation between the packing index and 2-Si of constituent clinopyroxene of olivine-rich inclusions, as well as the same relation of intrusive peridotite, was discussed by YAMAGUCHI (1964). He pointed out that the clinopyroxene of olivine-rich inclusions in basaltic rocks has high packing index and high 2-Si, whereas the clinopyroxene of intrusive peridotite has low packing index and low 2-Si, and accordingly he concluded that olivine-rich inclusions in basaltic rocks come from deeper part of the upper mantle than those of intrusive peridotite.

Recently in the study of the basic and ultrabasic inclusions in basaltic rocks from Hawaiian islands, WHITE (1966) has pointed out that olivine nephelinite is characterized by lhezolite inclusions, whereas alkali olivine basalt has inclusions of dunite, wehrlite and gabbro. Olivine tholeiite has a small amount of plagioclase peridotite. From these facts WHITE (1966) concluded that the origin of olivine-rich inclusions in basaltic rocks has more than one origin.

For the purpose of clarifying the problem, I have been engaged in the petrological and mineralogical studies of the basic and ultrabasic inclusions in basaltic rocks from northern Kyushu for these several years. The research has been concentrated on the chemical behaviour of constituent minerals, bulk chemical composition of olivine-rich inclusions, as well as bulk chemical composition of basaltic host rocks. In this paper the results of the studies are given with 
some petrogenetic discussions.

I wish to express my sincere thanks to Professor Sadakatsu TANEDA of Kyushu University for his kind guidance and criticism throughout this work, and for reading over the manuscript. I am much indebted to the late Professor Toru Tomita, Professor Tatsuro Matsumoto and Associate Professor Masaru YamaGUCHI of the same University for their advice and discussions with regard to this work. I am also indebted to former Professor Toyofumi Yoshimura, Professor Haruo SHIRozu and Mr. Masaharu OzAKI of Kyushu University for their advice about X-ray study.

Further, I wish to express my thanks to Professor Hisamichi Matsushita, Ryuzo TORIYAma and Associate Professor Hitoshi Momor for their advice and constant encouragement during this work. Thanks are also due to Professor Hisashi KUNo of Tokyo University and to Professor Yoshio OJI of Fukuoka University of Education for cooperation of my field survey and for kind discussion of basic and ultrabasic inclusions in basaltic rocks of Japan.

Financial expence of this study was partly defrayed by the Grant in Aid for Scientific Researches from the Ministry of Education, Japan.

\section{Geological setting and petrography of basic and ultrabasic inclusions in basaltic rocks}

The petrography of the Cenozoic basaltic rocks from San-in and northern Kyushu and geology of these areas have already been studied by several authors (AOKI, 1951; MATSUмото, 1960; OJI, 1960a, 1960b, 1961a, 1961b, 1962, 1964; YAMAGUCHI, 1964). Therefore in the present paper only the description is given for the samples used in this study.

The basic and ultrabasic inclusions occur in the basaltic rocks which are usually exposed in a small scale mostly near volcanic necks or eruption vents. Various rock types of basic and ultrabasic inclusions described in this paper are collected at the following localities in southwestern Japan (Fig. 1) :

1) Takashima island, Karatsu City

2) Kurose in Hakata Bay, Fukuoka City

3) Ogusoyama, Shimane Prefecture

At each locality, the samples have been collected from a selected single lava flow.

\section{A. Basic and ultrabasic inclusions from Takashima island}

Takashima is a small island in Karatsu Bay, with about $3 \mathrm{~km}$ in circumference, composed of pre-Tertiary granodiorite, granite and small dykes of lamplophyre and Pleistocene basaltic flows unconformably covering the granitic rocks. Thick beds of scoriaceous tuff and volcanic bombs are also found in the southwestern cliff of the island. The basalt flows commonly contain basic and ultrabasic inclusions, which are up to $20-30 \mathrm{~cm}$ in diameter, and also xenolithes of the basement rocks.

Modal abundance of basic and ultrabasic inclusions in basaltic rocks from this island has already been described by YAMAGUCHI (1964). The inclusions 


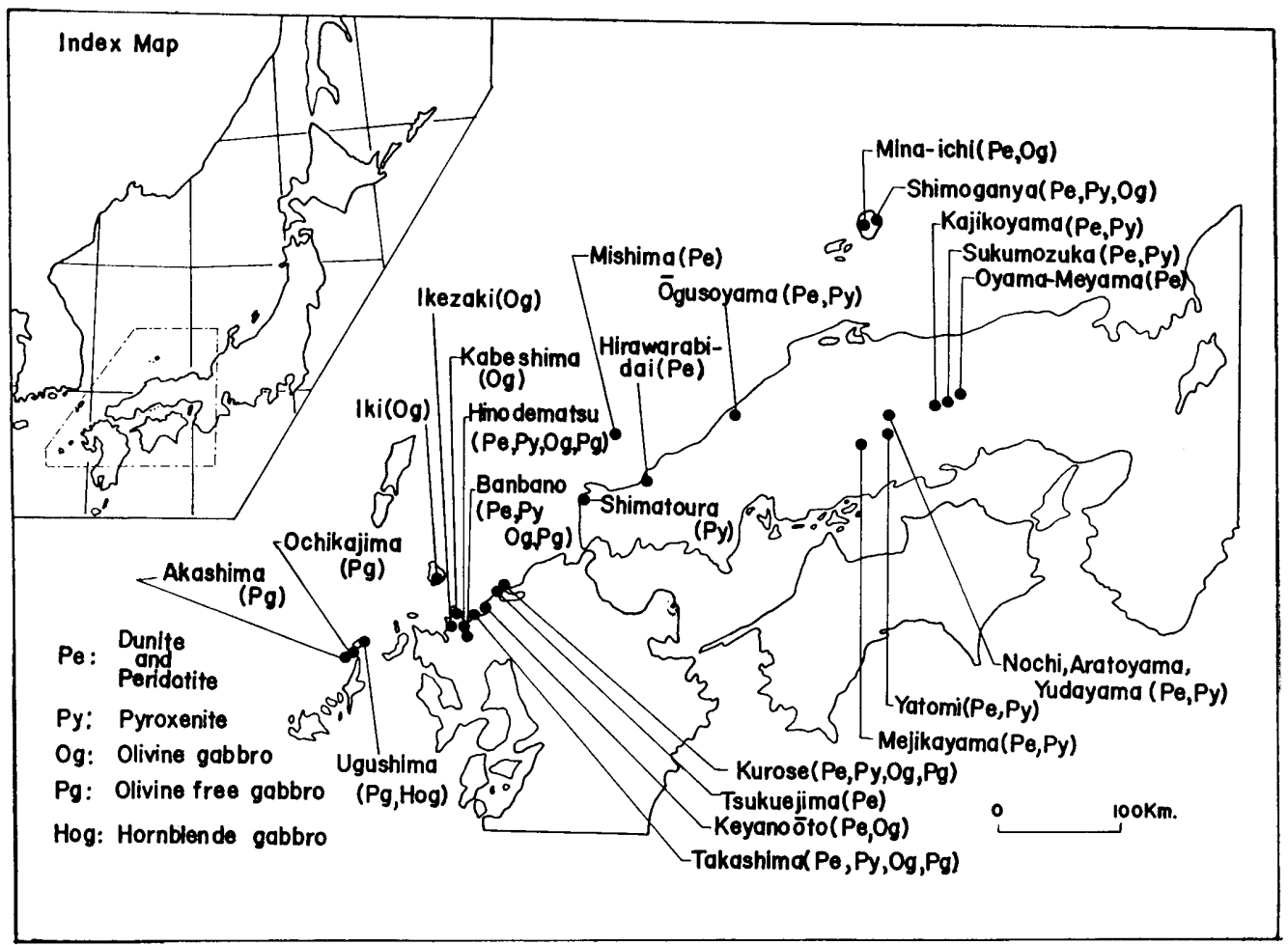

Fig. 1. Distribution of basic and ultrabasic inclusions in basaltic rocks from southwestern Japan, complied from AoYAMA (1942), HARUmoto (1951, 1952), OJI (1961a, 1961c) and YAMAGUCHI (1964).

have a wide range in modal composition from dunite through peridotite and pyroxenite to gabbro. In this paper they are divided into seven types as follows:

1 Dunite

2 Cr-diopside peridotite

3 Cr-pyroxenite

4 Anorthite-bearing Cr-pyroxenite

green type inclusion

5 Al-pyroxenite

6 Olivine gabbro

7 Olivine-free gabbro

black type inclusion

Dunite, Cr-diopside peridotite, Cr-pyroxenite and anorthite-bearing $\mathbf{C r}$ pyroxenite contain spring green to grass green pyroxenes. Olivine gabbro, olivine-free gabbro and Al-pyroxenite have pyroxenes of pitch black to brownish black varieties. Thus we can easily classify the inclusions into two groups, the green type and the black type as defined above.

Gigantic phenocrysts of pitch black augite and hypersthene (AOYAMA, 1942; IsHIBASHI, 1962) up to $6 \mathrm{~cm}$ in diameter are also found in the basalt (Plate 18, Figs. 1 and 2). A few crystals of kaersutite are found in the same basaltic lava flows (KUNo, 1964). In addition to the gigantic phenocrysts, there are smaller crystals of olivine and augite which appear to be ordinary phenocrysts of the basalt. 


\section{a) Dunite}

It consists essentially of olivine and chromite with a small amount of Cr-diopside and with or without Cr-enstatite (Plate 20, Figs. 5 and 6). The amount of chromite in dunite ranges from one to twenty per cent. The zonal band of chromite and olivine is always recognized. The chromite is euhedral with reddish color. The olivine in dunite is usually $0.8-4 \mathrm{~mm}$, sometimes reaches $2-3 \mathrm{~cm}$ in length. The crystals are fresh with light green color. In thin section olivine is transparent and homogeneous with some inclusions of chromite, rutile and some other oxide minerals. Translation lamellae are sometimes observed (Plate 24, Fig. 12).

b) Cr-diopside peridotite

This rock consists largely of Cr-diopside and olivine with a small amount of Cr-enstatite and chromite. The amount of olivine varies from about ten to ninety per cent. Some Cr-diopside peridotite shows distinct foliation or banded texture due to different concentration of olivine in layer of variable thickness ranging from a few milimeter to several centimeters. Olivine and orthopyroxene show undulated extinction and have translation lamellae, suggesting that the rock underwent the deformation in the solid state.

c) Cr-pyroxenite and anorthite-bearing Cr-pyroxenite

These rocks consist of a large amount of Cr-diopside and a small amount of Cr-enstatite (Plate 25, Fig. 23). Olivine may or may not be present. Spinel occurs in a small amount. In some Cr-pyroxenite, anorthite is present about five per cent in volume. Rhythmical layering of plagioclase and Cr-pyroxene is recognizable in some pyroxenite (Plate 19, Fig. 3). Dustic inclusions of rutile, chromite and some other oxide minerals are discernible in Cr-diopside or $\mathrm{Cr}$ enstatite.

\section{d) Al-pyroxenite}

This rock consists of pitch black Al-augite, Al-hypersthene and a small amount of olivine and aluminous spinel. Al-augite is somewhat rich in $\mathrm{TiO}_{2}$ and Al-hypersthene shows distinct pleochroism in thin section.

Spinel is dark green and/or almost translucent. Al-pyroxenite is often formed around dunite, Cr-diopside peridotite and Cr-pyroxenite as a reaction rim (Plate 24, Figs. 17 and 18). Both Al-augite and Al-hypersthene are partly decomposed at the margin of the crystals to fine-grained aggregate of olivine, augite, pigeonite, plagioclase and opaque minerals. The same phenomenon is observed in large phenocrystic augite and hypersthene (Plate 24, Figs. 19 and 20;

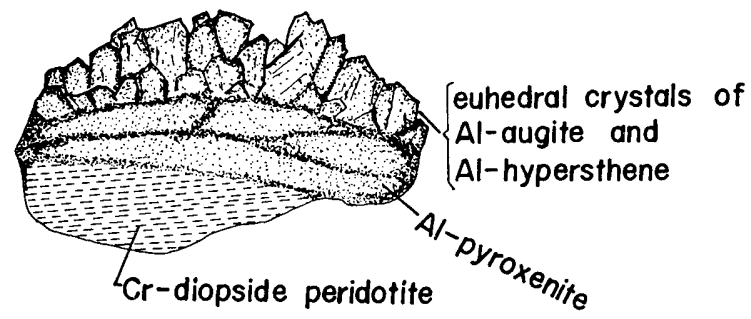

Fig. 2. A sketch showing the relation between Al-pyroxnite and $\mathrm{Cr}-$ diopside peridotite. 
Plate 23, Figs. 13 and 14).

Figure 2 shows the relation between peridotite and Al-pyroxenite; the core is peridotite, the outer zone is Al-pyroxenite. In the outermost part, euhedral crystals of Al-augite and Al-hypersthene are developed, having crystal faces of $\mathrm{a}(100), \mathrm{u}(111), \mathrm{m}(110), \mathrm{c}(001)$ and $\mathrm{b}(010)$.

\section{B. Gabbro and dunite in the basaltic rocks from Kurose in Hakata Bay, Fukuoka City}

Kurose near Genkai-jima island in Hakata Bay is the locality of pyroclastic rocks, scoriaceous rocks and basaltic flows. The basement is not exposed.

Basic and ultrabasic inclusions occur in basaltic lava flows and also in pyroclastic rocks. Banded gabbro and dunite are the most predominant. Crdiopside peridotite and Cr-pyroxenite are also found in the same basaltic rocks, although they are small in size and very scanty in amount.

a) Olivine gabbro and olivine-free gabbro

Gabbro shows wide variation in modal composition from allivalite to Alpyroxenite even within a single rock specimen due to the variable amount of plagioclase. Zonal arrangement of mafic and felsic minerals is common (Plate 19, Fig. 4).

In the dark part, Al-augite and Al-hypersthene are predominant, while in the white part, plagioclase is the main constituent. A small amount of aluminous spinel is also present together with or without olivine. Under the microscope, the spinel shows deep green color and euhedral outline. Myrmekite-like texture of spinel and pyroxene (symplectite ?) is observed.

b) Dunite

The dunite from Kurose shows the same mineralogical composition and mode of occurrence as the dunite from Takashima.

\section{Peridotite inclusions in the limburgite from Ogusoyama, Shimane Prefecture}

Ogusoyama is a small rise, about $15 \mathrm{~km}$ SSE of Hamada City in the western part of Shimane Prefecture. Limburgite occurs at the top of the hill with the thickness of about 60 meters, lying on granodiorite and Palaeozoic formations. Limburgite is gray to black in color and porphyritic with a large amount of olivine and clinopyroxene as phenocryst. The groundmass is composed of olivine, augite, magnetite, apatite and glass with or without sodic plagioclase. Dunite and peridotite inclusions up to $10 \mathrm{~cm}$ in diameter are found in the limburgite mass, and it is easy to collect them where the host rock has altered to soil by weathering. Most of my samples were collected in the altered limburgite near the top of the hill, but olivine-rich inclusions are fresh enough to be supplied for chemical analyses. In thin section olivine is strongly striated, shows wavy extinction, and sometimes has translation lamellae. Diopside and augite have pleochroism. Each constituent minerals of these inclusions have been affected at the margin by basaltic magma. Namely, olivine has changed to fine mosaic aggregate of olivine, plagioclase and pigeonite. Augite shows a spongy texture (YAMAGUCHI, 1964). 


\section{Optical properties of constituent minerals and modal composition of basic and ultrabasic inclusions}

The modal analyses of constituent minerals in basic and ultrabasic inclusions have been done by ordinary method of volumetry by using a line integrator, and the results are set in Table 2 and in Fig. 3. The optical properties of constituent minerals are shown in Table 1.

Table 1. Optical properties of constituent mineral in basic and ultrabasic inclusions and phenocrysts of basalts.

\begin{tabular}{|c|c|c|c|c|c|}
\hline No. & spinel & olivine & clinopyroxene & orthopyroxene & plagioclase \\
\hline 1 & $\begin{array}{l}\text { reddish } \\
\text { brown }\end{array}$ & $\begin{array}{l}\mathrm{Nx}=1.652 \\
\mathrm{Nz}=1.694\end{array}$ & $\begin{array}{l}\mathrm{Ny}=1.679-1.684 \\
2 \mathrm{Vz}=54\end{array}$ & $\begin{array}{l}\mathrm{Nx}=1.664 \\
\mathrm{Nz}=1.684\end{array}$ & non \\
\hline 2 & $\begin{array}{l}\text { reddish } \\
\text { brown }\end{array}$ & $\begin{array}{l}\mathrm{Nx}=1.648 \\
\mathrm{Nz}=1.693\end{array}$ & $\begin{array}{l}\mathrm{Ny}=1.677-1.682 \\
2 \mathrm{Vz}=53\end{array}$ & $\begin{array}{l}\mathrm{Nx}=1.664 \\
\mathrm{Nz}=1.681\end{array}$ & non \\
\hline 3 & $\begin{array}{l}\text { reddish } \\
\text { brown }\end{array}$ & $\begin{array}{l}\mathrm{Nx}=1.649 \\
\mathrm{Nz}=1.693\end{array}$ & $\begin{array}{l}\mathrm{Ny}=1.676-1.680 \\
2 \mathrm{Vz}=54\end{array}$ & non & non \\
\hline 4 & $\begin{array}{l}\text { reddish } \\
\text { brown }\end{array}$ & $\begin{array}{l}\mathrm{Nx}=1.646 \\
\mathrm{Nz}=1.692\end{array}$ & $\begin{array}{l}\mathrm{Ny}=1.677-1.682 \\
2 V \mathrm{z}=55\end{array}$ & $\begin{array}{l}\mathrm{Nx}=1.664 \\
\mathrm{Nz}=1.682\end{array}$ & non \\
\hline 5 & $\begin{array}{l}\text { reddish } \\
\text { brown }\end{array}$ & $\begin{array}{l}\mathrm{Nx}=1.650 \\
\mathrm{Nz}=1.694\end{array}$ & $\begin{array}{l}\mathrm{Ny}=1.676-1.682 \\
2 \mathrm{Vz}=54\end{array}$ & $\begin{array}{l}N x=1.662 \\
N z=1.681\end{array}$ & non \\
\hline 6 & brown & $\begin{array}{l}\mathrm{Nx}=1.657 \\
\mathrm{Nz}=1.704\end{array}$ & $\begin{array}{l}\mathrm{Ny}=1.682-1.686 \\
2 \mathrm{Vz}=48\end{array}$ & $\begin{array}{l}\mathrm{Nx}=1.665 \\
\mathrm{Nz}=1.683\end{array}$ & non \\
\hline 7 & non & $\begin{array}{l}\mathrm{Nx}=1.660 \\
\mathrm{Nz}=1.711\end{array}$ & $\begin{array}{l}\mathrm{Ny}=1.679-1.685 \\
2 \mathrm{Vz}=51\end{array}$ & $\begin{array}{l}\mathrm{Nx}=1.666 \\
\mathrm{Nz}=1.688\end{array}$ & non \\
\hline 8 & $\begin{array}{l}\text { reddish } \\
\text { brown }\end{array}$ & $\begin{array}{l}\mathrm{Nx}=1.653 \\
\mathrm{Nz}=1.698\end{array}$ & $\begin{array}{l}\mathrm{Ny}=1.683-1.689 \\
2 \mathrm{Vz}=54\end{array}$ & $\begin{array}{l}\mathrm{Nx}=1.663 \\
\mathrm{Nz}=1.684\end{array}$ & non \\
\hline 9 & $\begin{array}{l}\text { reddish } \\
\text { brown }\end{array}$ & $\begin{array}{l}\mathrm{Nx}=1.648 \\
\mathrm{Nz}=1.691\end{array}$ & $\begin{array}{l}\mathrm{Ny}=1.684-1.691 \\
2 \mathrm{Vz}=51\end{array}$ & non & non \\
\hline 10 & non & non & $\begin{array}{l}\mathrm{Ny}=1.680-1.685 \\
2 \mathrm{~V} z=50\end{array}$ & $\begin{array}{l}\mathrm{Nx}=1.671 \\
\mathrm{Nz}=1.692\end{array}$ & non \\
\hline 11 & $\begin{array}{l}\text { reddish } \\
\text { brown }\end{array}$ & non & $\begin{array}{l}\mathrm{Ny}=1.675-1.683 \\
2 \mathrm{Vz}=52\end{array}$ & non & non \\
\hline 12 & $\begin{array}{l}\text { reddish } \\
\text { brown }\end{array}$ & non & $\begin{array}{l}\mathrm{Ny}=1.683-1.688 \\
2 \mathrm{Vz}=52\end{array}$ & $\begin{array}{l}\mathrm{Nx}=1.671 \\
\mathrm{Nz}=1.692\end{array}$ & non \\
\hline 13 & non & non & $\begin{array}{l}\mathrm{Ny}=1.683-1.687 \\
2 \mathrm{Vz}=53\end{array}$ & $\begin{array}{l}\mathrm{Nx}=1.670 \\
\mathrm{Nz}=1.692\end{array}$ & non \\
\hline 14 & reddish & non & $\begin{array}{l}\mathrm{Ny}=1.683-1.686 \\
2 \mathrm{Vz}=56\end{array}$ & $\begin{array}{l}\mathrm{Nx}=1.676 \\
\mathrm{Nz}=1.701\end{array}$ & non \\
\hline 15 & $\begin{array}{l}\text { trans- } \\
\text { lucent }\end{array}$ & non & $\begin{array}{l}\mathrm{Ny}=1.692-1.702 \\
2 \mathrm{Vz}=54\end{array}$ & $\begin{array}{l}\mathrm{Nx}=1.675 \\
\mathrm{Nz}=1.702\end{array}$ & non \\
\hline 16 & $\begin{array}{l}\text { grass } \\
\text { green }\end{array}$ & non & $\begin{array}{l}\mathrm{Ny}=1.685-1.690 \\
2 \mathrm{Vz}=54\end{array}$ & $\begin{array}{l}\mathrm{Nx}=1.670 \\
\mathrm{Nz}=1.690\end{array}$ & $\begin{array}{l}\mathrm{Nx}=1.570 \\
\mathrm{Nz}=1.585\end{array}$ \\
\hline 17 & $\begin{array}{l}\text { greenish } \\
\text { brown }\end{array}$ & non & $\begin{array}{l}\mathrm{Ny}=1.684-1.689 \\
2 \mathrm{Vz}=53\end{array}$ & $\begin{array}{l}\mathrm{Nx}=1.677 \\
\mathrm{Nz}=1.693\end{array}$ & $\begin{array}{l}\mathrm{Nx}=1.554 \\
\mathrm{Nz}=1.569\end{array}$ \\
\hline 18 & $\begin{array}{l}\text { grass } \\
\text { green }\end{array}$ & non & $\begin{array}{l}\mathrm{Ny}=1.683-1.689 \\
2 \mathrm{Vz}=53\end{array}$ & $\begin{array}{l}\mathrm{Nx}=1.688 \\
\mathrm{Nz}=1.709\end{array}$ & $\begin{array}{l}\mathrm{Nx}=1.572 \\
\mathrm{Nz}=1.588\end{array}$ \\
\hline 19 & $\begin{array}{l}\text { trans- } \\
\text { lucent }\end{array}$ & $\begin{array}{l}\mathrm{Nx}=1.655 \\
\mathrm{Nz}=1.702\end{array}$ & $\begin{array}{l}\mathrm{Nx}=1.690-1.698 \\
2 \mathrm{Vz}=54\end{array}$ & $\begin{array}{l}\mathrm{Nx}=1.678 \\
\mathrm{Nz}=1.701\end{array}$ & $\begin{array}{l}\mathrm{Nx}=1.547 \\
\mathrm{Nz}=1.561\end{array}$ \\
\hline
\end{tabular}

As is clear in Figure 3, the basic and ultrabasic inclusions show a wide range in modal composition but their optical properties (Table 2) and chemical composition (Tables $9,10,14,16$ ) of each constituent minerals, such as olivine, 
Table 2. Modal analyses of basic and ultrabasic inclusions in basaltic rocks

\begin{tabular}{|c|c|c|c|c|c|c|c|}
\hline $\begin{array}{c}\text { Specimen } \\
\text { No. }\end{array}$ & spienl & olivine & clino-px. & ortho-px. & $\begin{array}{l}\text { plago- } \\
\text { clase }\end{array}$ & locality & rock type \\
\hline 1 & 3 & 94 & 3 & 0.1 & 0 & Takashima & \\
\hline 2 & 8 & 88 & 3 & 1.0 & 0 & Takashima & dunite \\
\hline 3 & 15 & 83 & 2 & 0 & 0 & Takashima & \\
\hline 4 & 1 & 83 & 8 & 8 & 0 & Takashima & \\
\hline 5 & 20 & 75 & 5 & 0.1 & 0 & Takashima & Cr-diopside \\
\hline 6 & 1 & 59 & 35 & 5 & 0 & Takashima & peridotite \\
\hline 7 & 1 & 30 & 59 & 10 & 0 & Ogusoyama & \\
\hline 8 & 4 & 8 & 75 & 13 & 0 & Takashima & \\
\hline 9 & 0.1 & 5 & 94.9 & 0 & 0 & Takashima & \\
\hline 10 & 0 & 0 & 96 & 4 & 0 & Kurose & \\
\hline 11 & 0.1 & 0 & 99.9 & 0 & 0 & Takashima & Cr-pyroxenite \\
\hline 12 & trace & 0 & 95 & 5 & 0 & Takashima & \\
\hline 13 & 0 & 0 & 90 & 10 & 0 & Takashima & \\
\hline 14 & 0.1 & 0 & 70 & 30 & 0 & Takashima & \\
\hline 15 & 2.5 & 0 & 82.5 & 15 & 0 & Takashima.. & Al-pyroxenite \\
\hline 16 & 0.1 & 0 & 75 & 20 & 4.9 & Takashima & $\begin{array}{l}\text { anortite-bearing } \\
\text { Cr-pyroxenite }\end{array}$ \\
\hline 17 & 0.1 & 0 & 40 & 20 & 33.9 & Takashima & Lahbro \\
\hline 18 & 2 & 0 & 23 & 25 & 50 & Kurose & Gabbro \\
\hline 19 & \multicolumn{5}{|c|}{ phenocryst minerals in basaltic rocks } & Takashima & Basalt \\
\hline
\end{tabular}

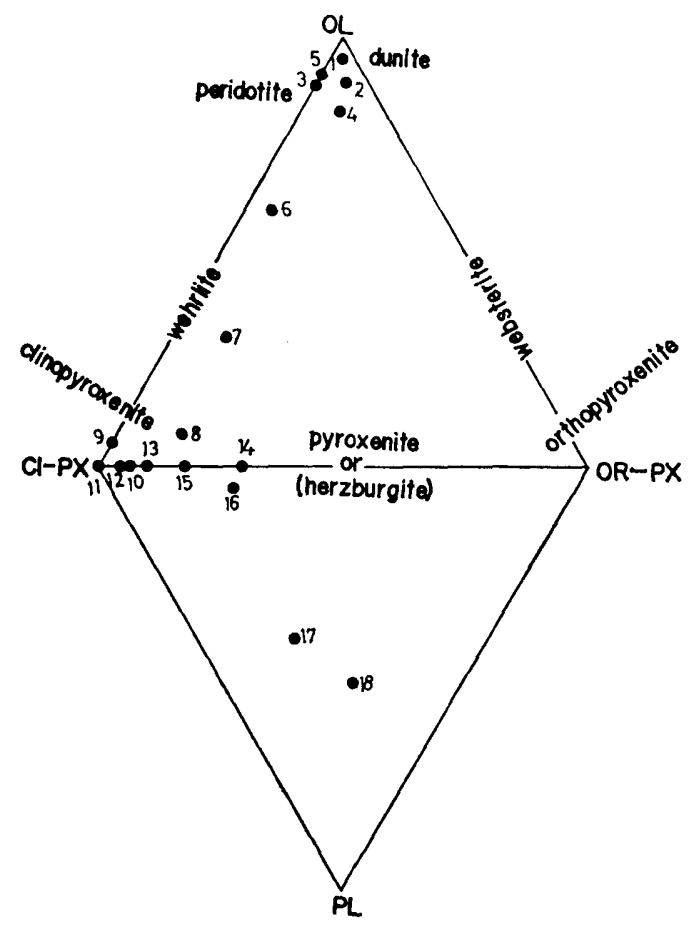

Fig. 3. Modal composition of basic and ultrabasic inclusions in basaltic rocks used for the present work. The content of spinel is exculded. 
orthopyroxene and clinopyroxene are not so different from a specimen to another, except in the case of Al-pyroxenite and some gabbros which are probably formed by the crystals accumulation of basaltic host magmas.

The modal frequency of basic and ultrabasic inclusions in basaltic rocks from southwestern Japan is shown in Table 3.

Table 3. Frequency of rock types of basic and ultrabasic inclusions in basaltic and limburgitic host rocks from southwestern Japan (Nos. 1-6, Yamaguchi 1964, Nos. 7 and 8 are new mesurments).

\begin{tabular}{|c|c|c|c|c|c|c|}
\hline No. & localities and host rock & dunite & peridotite & $\begin{array}{l}\text { pyro- } \\
\text { xenite }\end{array}$ & $\begin{array}{l}\text { olivine } \\
\text { gabbro }\end{array}$ & $\begin{array}{l}\text { olivine } \\
\text { free } \\
\text { gabbro }\end{array}$ \\
\hline 1 & $\begin{array}{l}\text { Shimoganya, Oki Is. } \\
\text { (olivine basalt) }\end{array}$ & 0 & 15 & 15 & 30 & 40 \\
\hline 2 & $\begin{array}{l}\text { Oyama and Meyama } \\
\text { (limburgite) }\end{array}$ & 0 & 100 & 0 & 0 & 0 \\
\hline 3 & $\begin{array}{l}\text { Sukumozuka and Kajikoyama } \\
\text { (limburgite) }\end{array}$ & 98 & 0 & 2 & 0 & 0 \\
\hline 4 & $\begin{array}{l}\text { Nochi and its environs } \\
\text { (picrite basalt) }\end{array}$ & 0 & 20 & 80 & 0 & 0 \\
\hline 5 & $\begin{array}{l}\text { Ogusoyama } \\
\text { (limburgite) }\end{array}$ & 15 & 80 & 5 & 0 & 0 \\
\hline 6 & $\begin{array}{l}\text { Kurose } \\
\text { (olivine basalt) }\end{array}$ & 70 & 1 & 1 & 0 & 28 \\
\hline 7 & $\begin{array}{l}\text { Takashima and its environs } \\
\text { (olivine basalt) }\end{array}$ & 40 & 32 & 14 & 2 & 12 \\
\hline 8 & $\begin{array}{l}\text { Ugushima and its environs } \\
\text { (olivine basalt) }\end{array}$ & 0 & 0 & 0 & 10 & 90 \\
\hline
\end{tabular}

\section{Chemical and X.ray analyses}

\section{A. Preparation of sample for chemical and X-ray studies}

For the purpose of clarifying the paragenesis, the chemical composition, and the distribution of elements of co-existing minerals within the same fragment, a single block about $1 \mathrm{Kg}$ to $2 \mathrm{Kg}$ in weight of basic and ultrabasic inclusion is treated by the following method.

As the marginal part of the inclusions had always been altered to some extent, the surface was completely peeled off. The block was crushed to the grain size of 100-150 mesh. The pulverized materials were washed with water and after that washed again with alcohol, and were dried. From the dry materials, the constituent minerals, such as spinel, olivine, orthopyroxene, clinopyroxene and plagioclase were separated by using Frantz isodynamic separator. By repeating this procedure, the pure sample of olivine, orthopyroxene, clinopyroxene and plagioclase were obtained.

In the separation of spinel, however, a small amount of olivine or some other silicate minerals still remained as impurities. To get a pure sample of spinel, therefore, the material was treated with mixture of perchroic and hydrofroric acids at temperature $70-80^{\circ} \mathrm{C}$ in platinum dish. By this treatment silicate minerals were completely dissolved and an almost pure sample of spinel was obtained. 
The phenocryst minerals in the basaltic rocks are larger enough for chemical analyses even with a single crystal. All of the analyses of phenocryst minerals in this study were done by using one colt of crystal, or several cleavage flakes of a single crystal.

\section{B. Method of chemical analyses of silicate minerals}

The ordinary method of silicate analyses described by HILLEBrand and LUNDEL (1929), WASHINGTON (1930) and IWASAKI et al. (1958) was modified a little by the writer in the present study. The scheme of analysis is shown in Table 4.

Table 4. The scheme of analysis for silicate minerals and rocks

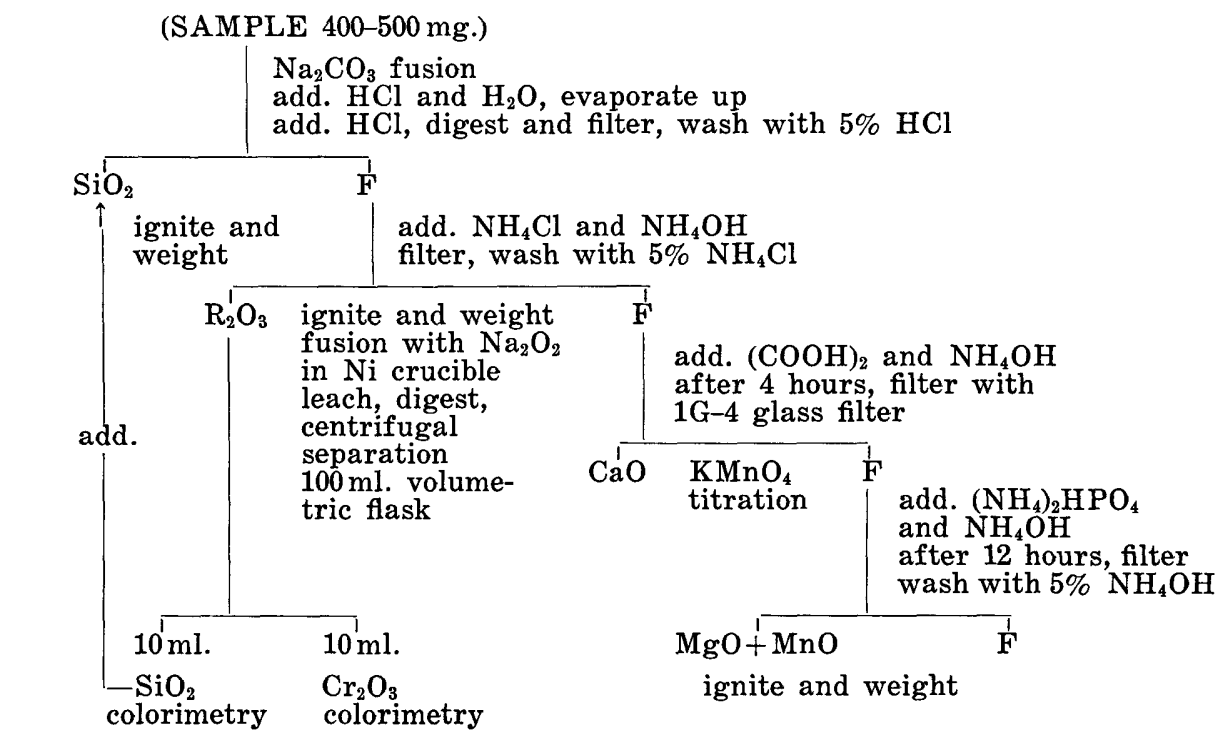

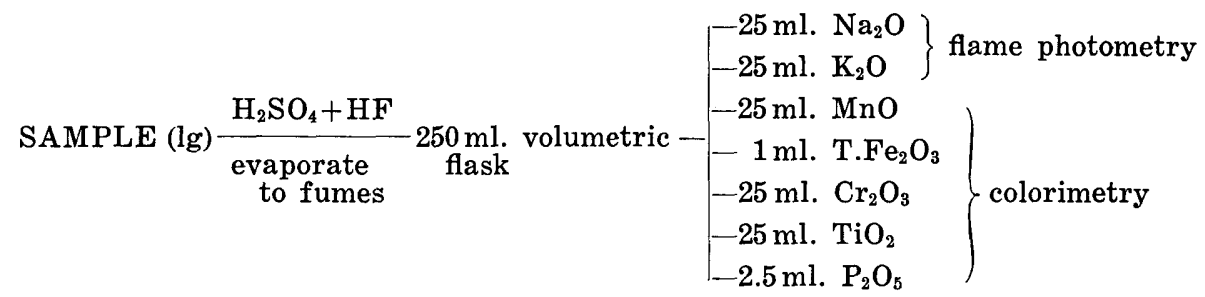

$$
\begin{aligned}
& \text { SAMPLE }(300-500 \mathrm{mg}) \frac{\mathrm{H}_{2} \mathrm{SO}_{4}+\mathrm{HF}}{\mathrm{KMnO}_{4} \text { titration }} \mathrm{FeO} \\
& \mathrm{Al}_{2} \mathrm{O}_{3}=\mathrm{R}_{2} \mathrm{O}_{3}-\left(\mathrm{P}_{2} \mathrm{O}_{5}+\mathrm{TiO}_{2}+\mathrm{Cr}_{2} \mathrm{O}_{3}+\mathrm{T} \cdot \mathrm{Fe}_{2} \mathrm{O}_{3}+\mathrm{SiO}_{2} \text { col. }\right) \\
& \mathrm{MgO}=(\mathrm{MgO}+\mathrm{MnO})_{\mathrm{wt} .}-\mathrm{MnO}_{\text {col. }} \text {. }
\end{aligned}
$$

C. Method of chemical analyses of spinel and chromite

We cannot break down spinel or chromite completely with sodium carbonate fusion. Potassium pyrosulphate or sodium peroxide fusion is undesirable because of subsequent treatment. Therefore a new method of analysis for spinel and chromite is required. Table 5 shows the new procedure of analysis for 
Table 5. The scheme of analysis for spinel and chromite

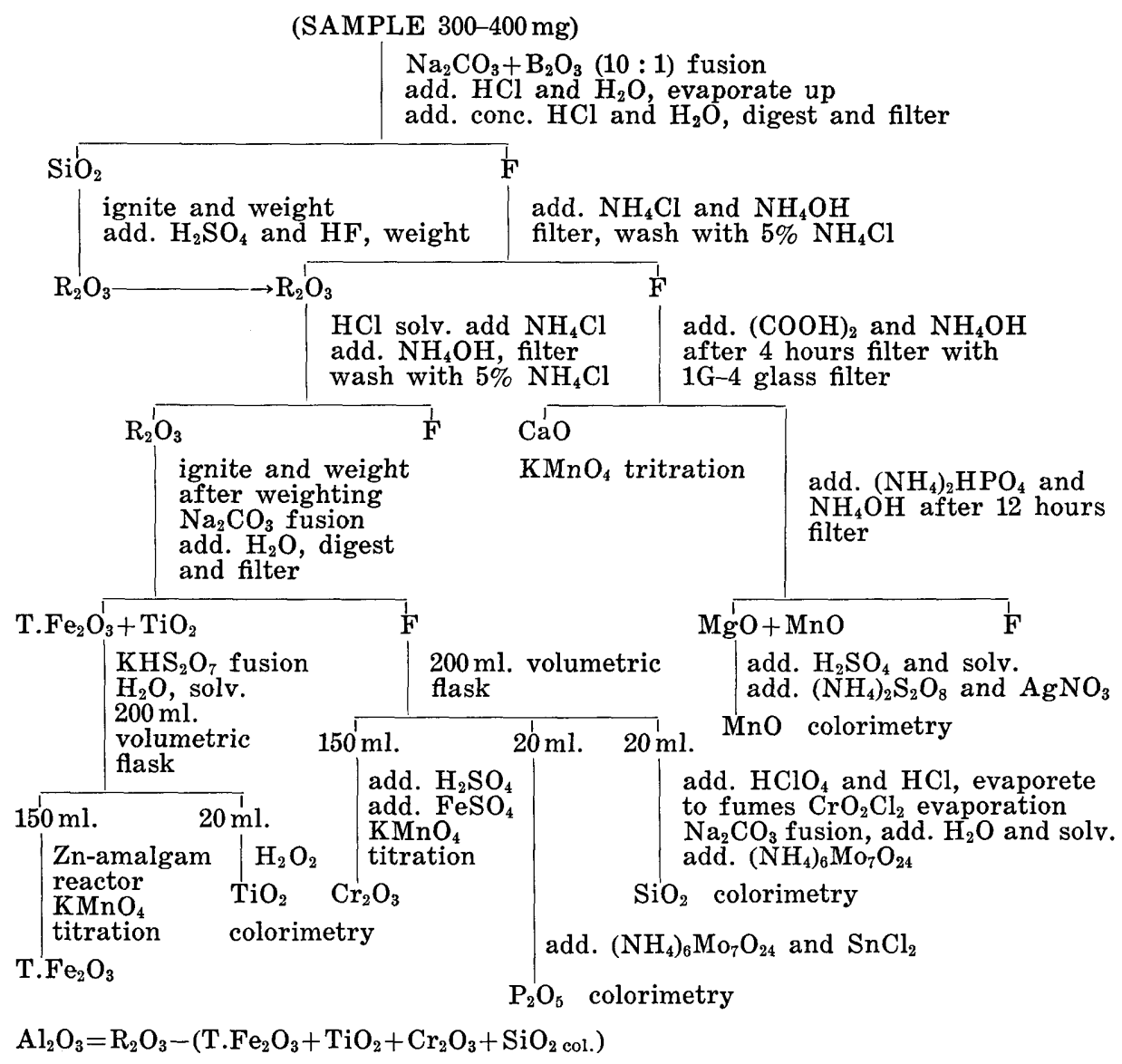

spinel and chromite devised by myself. This treatment was repeatedly tested by different analysts using the same sample and the results in good agreement with one another. The ferric iron content could not be determined by this method. Accordingly, FeO was calculated by the formula for spinel $R O: \mathrm{R}_{2} \mathrm{O}_{3}=1: 1$. In the present case $\mathrm{RO}=\mathrm{FeO}+\mathrm{MgO}+\mathrm{MnO}$ and $\mathrm{R}_{2} \mathrm{O}_{3}=\mathrm{Fe}_{2} \mathrm{O}_{3}+\mathrm{Cr}_{2} \mathrm{O}_{3}+\mathrm{Al}_{2} \mathrm{O}_{3}+$ $\mathrm{TiO}_{2}+\mathrm{SiO}_{2}$.

\section{List of analysed sample}

Chemical analyses have been made on the constituent minerals of eighteen basic and ultrabasic inclusions, and all of them are listed in Table 6 (Nos. 1-18).

Additional analyses are also made on the phenocrystic minerals (gigantic phenocrysts) of inclusion-bearing basalts, which are listed in the same table (Table 6, No. 19).

\section{E. Chemical composition of constituent minerals}

a) Spinel group

The spinel group has a number of end-members, but they occur in a limited 
Table 6. List of analyzed samples

\begin{tabular}{|c|c|c|c|c|c|}
\hline $\begin{array}{l}\text { specimens } \\
\text { No. }\end{array}$ & spinel & olivine & clino-px. & ortho-px. & plagioclase \\
\hline 1 & $1-\mathrm{A}$ & 1-B & $1-\mathrm{C}$ & - & - \\
\hline 2 & $2-\mathrm{A}$ & $2-B$ & $2-\mathrm{C}$ & - & - \\
\hline 3 & $3-\mathrm{A}$ & $3-B$ & - & - & - \\
\hline 4 & $4-\mathrm{A}$ & 4-B & $4-\mathrm{C}$ & $4-D$ & - \\
\hline 5 & $5-\mathrm{A}$ & $\begin{array}{l}5-B_{1} \\
5-B_{2}\end{array}$ & $5-\mathrm{C}$ & $5-\mathrm{D}$ & - \\
\hline 6 & - & $6-B$ & $\begin{array}{l}6-\mathrm{C}_{1} \\
6-\mathrm{C}_{2}\end{array}$ & - & - \\
\hline 7 & - & $7-\mathrm{B}$ & $7-\mathrm{C}$ & $7-\mathrm{D}$ & - \\
\hline 8 & - & $8-B$ & $8-\mathrm{C}$ & $8-D$ & - \\
\hline 9 & - & - & $9-\mathrm{C}$ & - & - \\
\hline 10 & - & - & $10-\mathrm{C}$ & - & - \\
\hline 11 & - & - & $11-\mathrm{C}$ & - & - \\
\hline 12 & - & - & $\begin{array}{l}12-\mathrm{C}_{1} \\
12-\mathrm{C}_{2}\end{array}$ & - & - \\
\hline 13 & - & - & $13-\mathrm{C}$ & $13-\mathrm{D}$ & - \\
\hline 14 & - & - & $14-\mathrm{C}$ & $14-\mathrm{D}$ & - \\
\hline 15 & $15-\mathrm{A}$ & - & $15-\mathrm{C}$ & $15-\mathrm{D}$ & - \\
\hline 16 & - & - & $16-\mathrm{C}$ & $16-\mathrm{D}$ & $16-\mathrm{E}$ \\
\hline 17 & - & - & $17-\mathrm{C}$ & $17-\mathrm{D}$ & $17-\mathrm{E}$ \\
\hline 18 & 18-A & - & $\begin{array}{l}18-C_{1} \\
18-C_{2}\end{array}$ & $18-\mathrm{D}$ & $18-\mathrm{E}$ \\
\hline 19 & - & $19-\mathrm{B}$ & $\begin{array}{l}19-C_{1} \\
19-C_{2} \\
19-C_{3} \\
19-C_{4} \\
19-C_{5}\end{array}$ & - & $\begin{array}{l}19-\mathbf{E}_{1} \\
19-\mathbf{E}_{2}\end{array}$ \\
\hline
\end{tabular}

Specimen numbers are the same as in Table 1.

extent in natural rocks. The principal end-members of spinel group discussed in this paper are tabulated as follows:

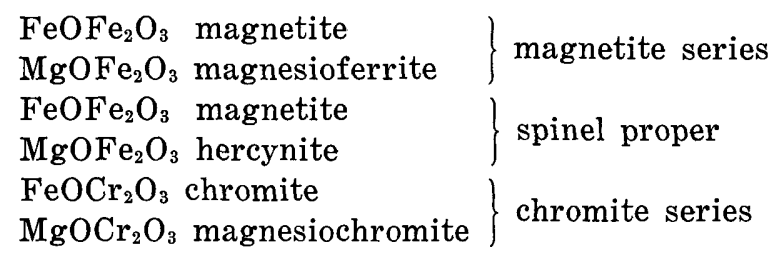

1) Chemical composition of spinel group

Table 7 shows the results of chemical analyses of spinels in the basic and ultrabasic inclusions in basaltic rocks. Two analyses of chromites (Nos. 1 and 2) in serpentinite from Shigiyama Mine, Ehime Prefecture, Japan are shown for comparison. The host rocks of Nos. 3 and 4 are dunites, Nos. 5 to 7 are peridotites and No. 8 is aluminous pyroxenite which is formed around peridotite inclusions (Fig. 2). They are collected from Takashima island. No. 9 is separated from banded gabbro from Kurose. 
Table 7. Chemical composition and lattice constant of spinels in the

\begin{tabular}{|c|c|c|c|c|}
\hline No. & 1 & 2 & $\stackrel{3}{(1-A)}$ & $\begin{array}{c}4 \\
(2-A)\end{array}$ \\
\hline $\mathrm{SiO}_{2}$ & 0.04 & 0.30 & 0.01 & 0.62 \\
\hline $\mathrm{TiO}_{2}$ & 0.50 & 0.50 & 0.60 & 0.69 \\
\hline $\mathrm{Al}_{2} \mathrm{O}_{3}$ & 6.63 & 5.46 & 9.01 & 8.24 \\
\hline $\mathrm{Cr}_{2} \mathrm{O}_{3}$ & 56.00 & 55.81 & 48.17 & 47.60 \\
\hline $\mathrm{Fe}_{2} \mathrm{O}_{3}$ & 10.40 & 12.03 & 16.05 & 16.63 \\
\hline $\mathrm{FeO}$ & 14.11 & 13.15 & 11.80 & 12.22 \\
\hline $\mathrm{MnO}$ & 0.04 & 0.15 & 0.30 & 0.12 \\
\hline $\mathrm{MgO}$ & 12.54 & 12.89 & 14.41 & 14.27 \\
\hline $\mathrm{CaO}$ & tr. & tr. & 0.01 & tr. \\
\hline $\mathrm{Na}_{2} \mathrm{O}$ & nd. & nd. & nd. & nd. \\
\hline $\mathrm{K}_{2} \mathrm{O}$ & - & - & - & - \\
\hline $\mathrm{P}_{2} \mathrm{O}_{5}$ & tr. & tr. & 0.01 & tr. \\
\hline Total & 100.26 & 100.29 & 100.37 & 100.39 \\
\hline $\mathrm{Si}$ & -1 & 0.050 & -1 & 0.155 \\
\hline $\mathrm{Ti}$ & 0.095 & 0.095 & 0.126 & 0.140 \\
\hline $\mathrm{Al}$ & $2.063\} 15.901$ & $1.713\} 15.876$ & $2.764 \succ 16.018$ & $2.480\} 15.703$ \\
\hline $\mathrm{Cr}$ & 11.680 & 11.639 & 9.956 & 9.796 \\
\hline $\mathrm{Fe}^{+3}$ & $2.063)$ & $2.379)$ & 3.172 & $3.132)$ \\
\hline $\mathrm{Fe}$ & $4.983)$ & $2.902)$ & 2.575 & $2.743)$ \\
\hline $\mathrm{Mn}$ & $-\} 8.094$ & $0.046\} 8.054$ & $0.063\} \quad 7.914$ & $0.016\} \quad 8.292$ \\
\hline $\mathrm{Mg}$ & 3.111 & 5.106 & 5.276 & 5.533 \\
\hline $0=32.000$ & $a_{0}=8.342 \mathrm{~A}$ & $\mathrm{a}_{0}=8.342 \AA$ & $a_{0}=8.341 \AA$ & $\mathrm{a}_{0}=8.341 \AA$ \\
\hline
\end{tabular}

No. 1 and No. 2 are in the serpentinite from Shigiyama Mine.

No. 3 and No. 4 are in the dunite from Takashima.

No. 5 is in the Cr-diopside peridotite from Takashima.

No. 6 is in the dunite from Takashima.

Atomic ratios calculated from the formula of spinel on the basis of $\mathrm{O}=32.000$ are shown in Table 7. The $\mathrm{Al}_{2} \mathrm{O}_{3}-\mathrm{Cr}_{2} \mathrm{O}_{3}-\mathrm{Fe}_{2} \mathrm{O}_{3}$ ratios are plotted on the triangular diagram as shown in Figure 4. As is clearly shown in this figure, the spinels in serpentinite are comparatively rich in $\mathrm{Cr}_{2} \mathrm{O}_{3}$ (chromite-picrochromite), and the $\mathrm{Cr}_{2} \mathrm{O}_{3}$ content gradually decreases its amount from dunite through peridotite to pyroxenite. The compositional change of host rocks is reflected to the gradual replacement among $\mathrm{Cr}_{2} \mathrm{O}_{3}, \mathrm{Al}_{2} \mathrm{O}_{3}$ and $\mathrm{Fe}_{2} \mathrm{O}_{3}$ contents in the chromitespinel series. On the other hand, it is noticeable that the spinels in Al-pyroxenite and gabbro are rich in $\mathrm{Al}_{2} \mathrm{O}_{3}$ (hercynite-spinel proper). $\mathrm{Al}_{2} \mathrm{O}_{3}$ is replaced only by $\mathrm{Fe}_{2} \mathrm{O}_{3}$ in spinel-magnetite series. Therefore the spinels in Al-pyroxenite and gabbro tend to have their compositions close to magnetite-spinel composition line (Fig. 2).

Extensive studies of oxide minerals in volcanic rocks from Japan were undertaken by IWASAKI and KATSURA (1950). They have concluded that the 
basic and ultrabasic inclusions in basaltic rocks (anal. K. Ishibashi)

\begin{tabular}{|c|c|c|c|c|}
\hline $\begin{array}{c}5 \\
(5-\mathrm{A})\end{array}$ & $\begin{array}{c}6 \\
(3-A)\end{array}$ & $\stackrel{7}{(4-A)}$ & $\stackrel{8}{8}(18-A)$ & $\stackrel{9}{(15-A)}$ \\
\hline 0.23 & 0.58 & 0.51 & 0.25 & 0.22 \\
\hline 0.59 & 0.64 & 0.38 & 0.41 & 0.81 \\
\hline 11.49 & 12.84 & 10.01 & 49.26 & 42.73 \\
\hline 46.16 & 42.05 & 30.97 & 5.05 & 1.04 \\
\hline 14.40 & 16.61 & 34.65 & 15.20 & 24.00 \\
\hline 11.30 & 13.58 & 4.10 & 11.30 & 15.19 \\
\hline 0.49 & 0.03 & 0.07 & 0.40 & 0.43 \\
\hline 15.02 & 14.10 & 19.57 & 18.59 & 15.48 \\
\hline tr. & tr. & tr. & tr. & tr. \\
\hline nd. & nd. & nd. & nd. & nd. \\
\hline- & nd. & nd. & nd. & nd. \\
\hline $\begin{array}{r}\text { tr. } \\
99.68\end{array}$ & $\begin{array}{r}\operatorname{tr} . \\
100.43\end{array}$ & $\begin{array}{r}\text { tr. } \\
100.26\end{array}$ & $\begin{array}{r}\operatorname{tr} . \\
100.46\end{array}$ & $\begin{array}{r}\text { tr. } \\
99.90\end{array}$ \\
\hline 0.062 & \multirow{5}{*}{15.698} & \multirow{5}{*}{15.790} & \multirow{5}{*}{15.879} & 0.054 \\
\hline \multirow{4}{*}{15.816} & & & & \multirow{3}{*}{15.811} \\
\hline & & & & \\
\hline & & & & \\
\hline & & & & $4.068)$ \\
\hline \multirow[b]{2}{*}{8.185} & \multirow{3}{*}{8.274} & \multirow{3}{*}{8.213} & 2.038 & 2.861 \\
\hline & & & 8.114 & $0.081\} \quad 8.190$ \\
\hline 5.675 & & & 5.999 & 5.248 \\
\hline $\mathrm{a}_{0}=8.299 \AA$ & $\mathrm{a}_{0}=8.298 \AA$ & $a_{0}=8.204 \AA$ & $a_{0}=8.119_{5} \AA$ & $\mathrm{a}_{0}=8.342 \AA$ \\
\hline
\end{tabular}

No. 7 is in the Cr-diopside peridotite from Takashima.

No. 8 is in the banded gabbro from Kurose.

No. 9 is in the Al-pyroxenite from Takashima.

oxide minerals of volcanic rocks are mostly of magnetite-ilmenite composition with less than two percent of $\mathrm{Al}_{2} \mathrm{O}_{3}$ and $\mathrm{Cr}_{2} \mathrm{O}_{3}$.

Recently AOKI (1966) has reported a large phenocrysts of magnetite which has a higher content of $\mathrm{Al}_{2} \mathrm{O}_{3}$ in trachyandesite from Madara-jima island, about $15 \mathrm{~km}$ northwest of Takashima island. He has demonstrated that the solubility of $(\mathrm{Mg}, \mathrm{Fe}) \mathrm{Al}_{2} \mathrm{O}_{4}$ molecule in magnetite is increased with increasing pressure. Some basalts from northern Kyushu contain large phenocrysts of magnetite, the content of $\mathrm{Al}_{2} \mathrm{O}_{3}$ reaches more than eight per cent (ISHIBASHI unpublished data). The compositional range of natural spinels in various kinds of host rocks is summarized and is shown in Fig. 5. From this figure, immiscible gaps are conceivable among the chromite-spinel-magnetite series in the igneous and metamorphic rocks.

WARSHAW and KEITH (1954) showed experimentally that complete solid solution exists between $\mathrm{MgCr}_{2} \mathrm{O}_{4}$ and $\mathrm{MgAl}_{2} \mathrm{O}_{4}$ at the temperature between $510^{\circ} \mathrm{C}$ 


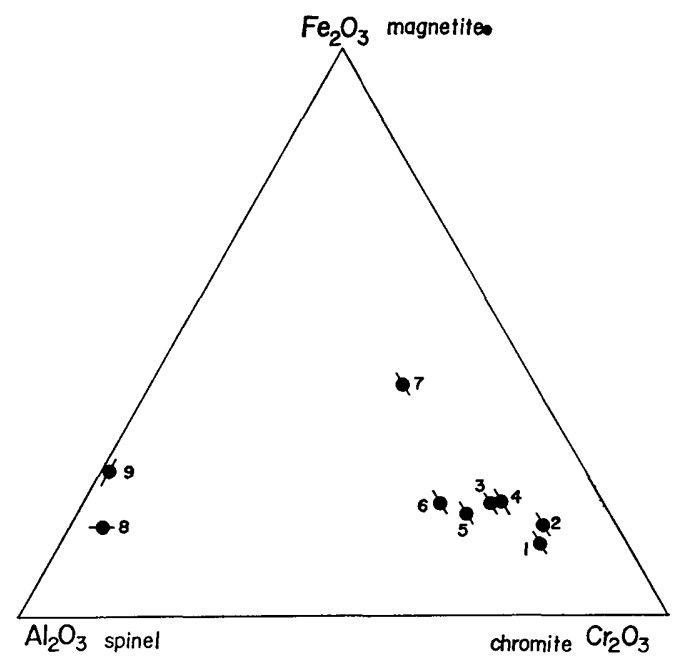

Fig. 4. Anelyzed spinels are plotted in $\mathrm{Cr}_{2} \mathrm{O}_{3}-\mathrm{Al}_{2} \mathrm{O}_{3}-\mathrm{Fe}_{2} \mathrm{O}_{3}$ triangular diagram. The number is the same as in Table 4.

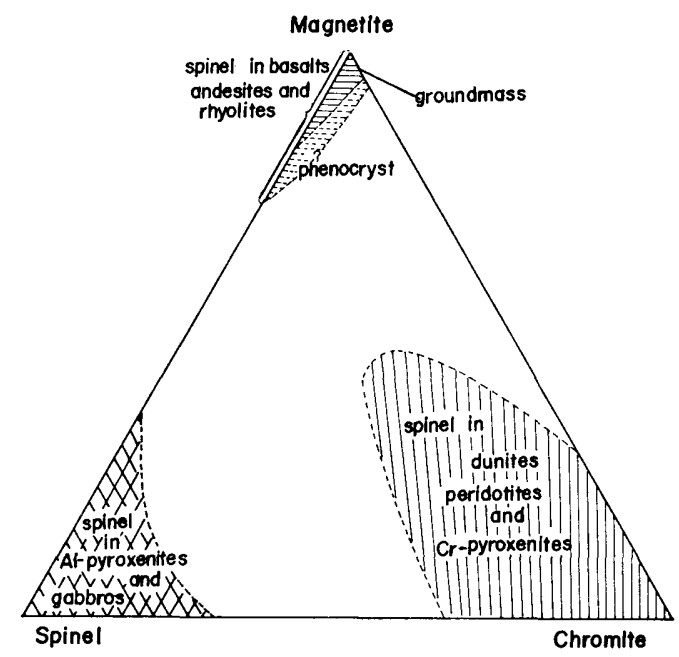

Fig. 5. The compositional relation of spinels and their host rocks.

and the solidus. WincheLL (1951) suggested that in natural spinel group, Fe replaces $\mathrm{Al}$ or $\mathrm{Cr}$ probably to a limited extent whereas $\mathrm{Al}$ can be replaced by $\mathrm{Cr}$ in any proportion. Accordingly, the spinel group includes two minerals; the spinel and the magnetite. The relation between spinel group and other silicate minerals will be discussed in another chapter.

2) X-ray analyses of spinel group

$X$-ray powder diffraction patterns of the spinels from Takashima island together with those from some other localities have been obtained by X-ray diffractometer using silicon as an internal standard. The lattice constants for these spinels are calculated from X-ray powder pattern. The result is shown 
Table 8. X-ray powder data of chromite from Takashima (Table 7, No. 6)

\begin{tabular}{|c|c|c|c|c|c|c|}
\hline $2 \theta_{\mathrm{obs}}$ & $\mathrm{d} \AA$ & Qobs. & $\mathrm{Q}_{\text {calc }}$ & $\begin{array}{l}Q_{\text {obs. }}-Q_{\text {calc. }} \\
\times 10^{-4}\end{array}$ & hkl & I \\
\hline 95.94 & 1.0370 & 0.9299 & 0.9295 & 4 & 800 & 3 \\
\hline 90.92 & 1.0807 & 0.8562 & 0.8569 & -7 & $\begin{array}{l}553 \\
731\end{array}$ & 3 \\
\hline 74.98 & 1.2656 & 0.6243 & 0.6245 & -2 & 533 & 2 \\
\hline 63.40 & 1.4658 & 0.4654 & 0.4648 & 6 & $\begin{array}{l}400 \\
222\end{array}$ & 5 \\
\hline 57.70 & 1.5963 & 0.3924 & 0.3921 & 3 & $\begin{array}{l}333 \\
511\end{array}$ & 5 \\
\hline 54.10 & 1.6937 & 0.3486 & 0.3485 & 1 & 422 & 3 \\
\hline 43.60 & 2.074 & 0.2325 & 0.2324 & 1 & 400 & 7 \\
\hline 35.82 & 2.505 & 0.1594 & 0.1597 & -3 & 311 & 10 \\
\hline 30.44 & 2.934 & 0.1162 & 0.1161 & 1 & 220 & 4 \\
\hline 18. 46 & 4.802 & 0.0434 & 0.0435 & -1 & 111 & 4 \\
\hline
\end{tabular}

Unite cell dimension, $\mathrm{a}_{0}=8.297_{7} \AA$. Conditions: $\mathrm{CuK} \alpha$, Ni-filter, $35 \mathrm{KV}$., $15 \mathrm{~mA}$. Scanning speed, $1 / 2 \mathrm{deg} . / \mathrm{min}$. Chart speed, $10 \mathrm{~mm} / \mathrm{min}$. Slits, $1-1-0.2$. Time constant, 4 seconds. Count full scale 1000 cts./sec.

in Table 7 together with their chemical compositions. As an example, a detailed result of X-ray analysis is given in Table 8.

The relation of unit cell dimension and chemical composition is in good agreement with those of previous works (Stevens, 1944; Kitahara, 1954).

b) Olivine

1) Chemical composition of olivine

Olivine is one of the main minerals of dunite, peridotite and some kinds of gabbros. Chemical compositions of olivines in these kinds of inclusions are set in Table 9. The atomic ratios calculated on the basis of $0=4.000$ are also shown in the same table.

No remarkable chemical differences among various rock types of inclusions are observed. The Fa contents of olivine range from $\mathrm{Fa}_{9}-\mathrm{Fa}_{16}$, the compositional range is in good accordance with the results of Ross et al. (1954), WILSHIRE and BiNNs (1961) and WHITE (1966).

2) NiO content in olivine

NiO content in olivine is determined with SANDEL's method (1950) using dimethylglyoxim procedure. The results of analyses are shown in Table 9.

RINGW00D (1955) has pointed out that in the system of $\mathrm{Ni}_{2} \mathrm{SiO}_{4}$ and $\mathrm{Mg}_{2} \mathrm{SiO}_{4}$ the system is binary from $\mathrm{Mg}_{2} \mathrm{SiO}_{4}$ to $\mathrm{MgNiSiO}_{4}$, but between $\mathrm{MgNiSiO}_{4}$ and $\mathrm{Ni}_{2} \mathrm{SiO}_{4}$ the crystal melts incongruently with precipitation of $\mathrm{NiO}$.

Olivine from basic and ultrabasic inclusions always contains up to eight per cent of $\mathrm{Fe}_{2} \mathrm{SiO}_{4}$ molecule which is capable of lowering the crystallization temperature. In such a condition $\mathrm{Ni}_{2} \mathrm{SiO}_{4}$ (m.p. $1640^{\circ} \mathrm{C}$ ) may be crystallized into the olivine solid solution.

Figure 6 shows the relation of $\mathrm{MgO}$ and $\mathrm{NiO}$ contents in olivines from northern Kyushu. The contents in olivines from Hayachine, Miyamori, Horoman (ONUKI, 1965) and Mt. Albert (JAMBOR and SMITH, 1964) are also plotted for comparison. 
Table 9. Chemical composition of olivines (anal. K. Ishibashi)

\begin{tabular}{|c|c|c|c|c|c|c|c|c|c|c|}
\hline No. & $\begin{array}{c}1 \\
(7-B)\end{array}$ & $\stackrel{2}{2}$ & $\stackrel{3}{\left(5-B_{1}\right)}$ & $\stackrel{4}{\left(5-B_{2}\right)}$ & $\begin{array}{c}5 \\
(2-B)\end{array}$ & $\begin{array}{c}6 \\
(1-B)\end{array}$ & $(\stackrel{7}{8-B)}$ & $\begin{array}{c}8 \\
(3-B)\end{array}$ & $\underset{(4-B)}{9}$ & $\begin{array}{c}10 \\
(19-B)\end{array}$ \\
\hline $\mathrm{SiO}_{2}$ & 38.66 & 38.75 & 39.11 & 39.51 & 39.75 & 39.94 & 39.99 & 40.25 & 40.26 & 40.30 \\
\hline $\mathrm{TiO}_{2}$ & 0.01 & 0.01 & 0.01 & 0.01 & 0.01 & 0.01 & 0.01 & 0.01 & 0.01 & 0.08 \\
\hline $\mathrm{Al}_{2} \mathrm{O}_{3}$ & 0.62 & 0.32 & 0.61 & 0.48 & 0.02 & 0.47 & 0.36 & 0.11 & 0.04 & 0.55 \\
\hline $\mathrm{Cr}_{2} \mathrm{O}_{3}$ & 0.09 & nd. & 0.03 & 0.06 & 0.07 & 0.05 & 0.04 & 0.05 & 0.07 & 0.03 \\
\hline $\mathrm{Fe}_{2} \mathrm{O}_{3}$ & 2.40 & 1.46 & 1.37 & 2.14 & 1.17 & 1.68 & 1.48 & 0.65 & 1.55 & 2.76 \\
\hline $\mathrm{FeO}$ & 11.89 & 10.50 & 8.44 & 8.40 & 8.86 & 7.11 & 9.11 & 9.68 & 8.45 & 7.99 \\
\hline $\mathrm{NiO}$ & 0.11 & nd. & 0.11 & 0.25 & 0.51 & 0.14 & 0.47 & 0.48 & 0.13 & 0.13 \\
\hline $\mathrm{MnO}$ & 0.21 & 0.15 & 0.10 & 0.08 & 0.16 & 0.14 & 0.16 & 0.16 & 0.15 & 0.11 \\
\hline $\mathrm{MgO}$ & 45.65 & 48.50 & 49.92 & 49.28 & 49.32 & 49.95 & 48.57 & 48.53 & 49.12 & 48.10 \\
\hline $\mathrm{CaO}$ & 0.01 & 0.00 & 0.02 & 0.01 & 0.01 & 0.01 & 0.01 & 0.01 & 0.01 & 0.01 \\
\hline $\mathrm{Na}_{2} \mathrm{O}$ & 0.04 & 0.09 & 0.04 & 0.03 & 0.01 & 0.05 & 0.03 & 0.01 & 0.02 & 0.03 \\
\hline $\mathrm{K}_{2} \mathrm{O}$ & 0.00 & 0.00 & 0.00 & 0.00 & 0.00 & 0.00 & 0.00 & 0.00 & 0.00 & 0.00 \\
\hline $\mathrm{H}_{2} \mathrm{O}^{+}$ & 0.15 & nd. & 0.06 & 0.03 & 0.03 & 0.06 & 0.02 & 0.04 & 0.09 & 0.03 \\
\hline $\mathrm{H}_{2} \mathrm{O}^{-}$ & 0.03 & nd. & 0.03 & 0.04 & 0.02 & 0.04 & 0.03 & 0.02 & 0.05 & 0.02 \\
\hline Total & 99.87 & 99.78 & 99.85 & 100.32 & 99.94 & 99.65 & 100.28 & 100.00 & 99.95 & 100.14 \\
\hline $\mathrm{Si}$ & 0.968 & 0.961 & 0.969 & 0.968 & 0.975 & 0.974 & 0.979 & 0.992 & 0.985 & 0.978 \\
\hline $\mathrm{Al}$ & 0.018 & 0.009 & 0.018 & 0.015 & - & 0.015 & 0.012 & 0.003 & - & 0.017 \\
\hline $\mathrm{Cr}$ & 0.003 & - & - & 0.003 & 0.003 & 0.003 & - & 0.003 & 0.003 & - \\
\hline $\mathrm{Fe}^{+3}$ & - & - & - & - & - & - & - & - & - & - \\
\hline $\mathrm{Ti}$ & - & - & - & - & - & - & - & - & - & - \\
\hline $\mathrm{Al}$ & - & - & - & - & - & - & - & - & - & - \\
\hline $\mathrm{Cr}$ & - & - & - & - & - & - & - & - & - & - \\
\hline $\mathrm{Fe}^{+3}$ & 0.045 & 0.027 & 0.027 & 0.041 & 0.024 & 0.032 & 0.029 & 0.012 & 0.029 & 0.053 \\
\hline $\mathrm{Ti}$ & - & - & - & - & - & - & - & - & - & 0.001 \\
\hline $\mathrm{Fe}$ & 0.284 & 0.271 & 0.174 & 0.164 & 0.182 & 0.145 & 0.185 & 0.198 & 0.173 & 0.182 \\
\hline $\mathrm{Ni}$ & 0.002 & - & 0.001 & 0.006 & 0.010 & 0.001 & 0.010 & 0.010 & 0.001 & 0.001 \\
\hline $\mathrm{Mn}$ & 0.005 & 0.003 & 0.001 & 0.002 & 0.003 & 0.003 & 0.004 & 0.003 & 0.003 & 0.001 \\
\hline $\mathrm{Mg}$ & 1.715 & 1.803 & 1.817 & 1.809 & 1.914 & 1.826 & 1.781 & 1.778 & 1.803 & 1.752 \\
\hline $\mathrm{Mg}$ & - & - & - & - & - & - & - & - & - & - \\
\hline $\mathrm{Ca}$ & - & - & - & - & - & - & - & - & - & - \\
\hline $\mathrm{Na}$ & - & 0.003 & - & - & - & - & - & - & - & - \\
\hline K & - & - & - & - & - & - & - & - & - & - \\
\hline \multicolumn{11}{|c|}{$0=4.000$} \\
\hline Fo & 84 & 86 & 90 & 90 & 90 & 91 & 89 & 89 & 90 & 88 \\
\hline $\mathrm{Fa}$ & 16 & 14 & 10 & 10 & 10 & 9 & 11 & 11 & 10 & 12 \\
\hline
\end{tabular}




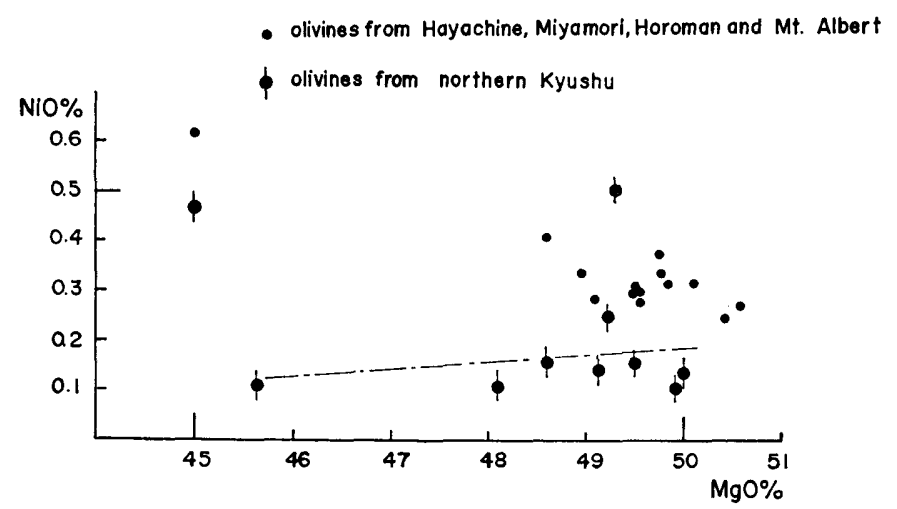

Fig. 6. The $\mathrm{NiO}$ content in olivines.

c) Clinopyroxene

1) Chemical composition of clinopyroxene

The results of chemical analyses of clinopyroxenes from basic and ultrabasic inclusions together with the atomic ratios calculated on the basis of $0=6.000$ are given in Table 10. Among twenty-five samples, ten are co-existing with orthopyroxene. As is shown in Table 10, they are augite and diopsite-salite in compositions. The clinopyroxene in Al-pyroxenite and gabbro have higher content of $\mathrm{Al}_{2} \mathrm{O}_{3}, \mathrm{FeO}$ and $\mathrm{TiO}_{2}$ and a lower content of $\mathrm{Cr}_{2} \mathrm{O}_{3}$ than that in dunite and peridotite inclusions.

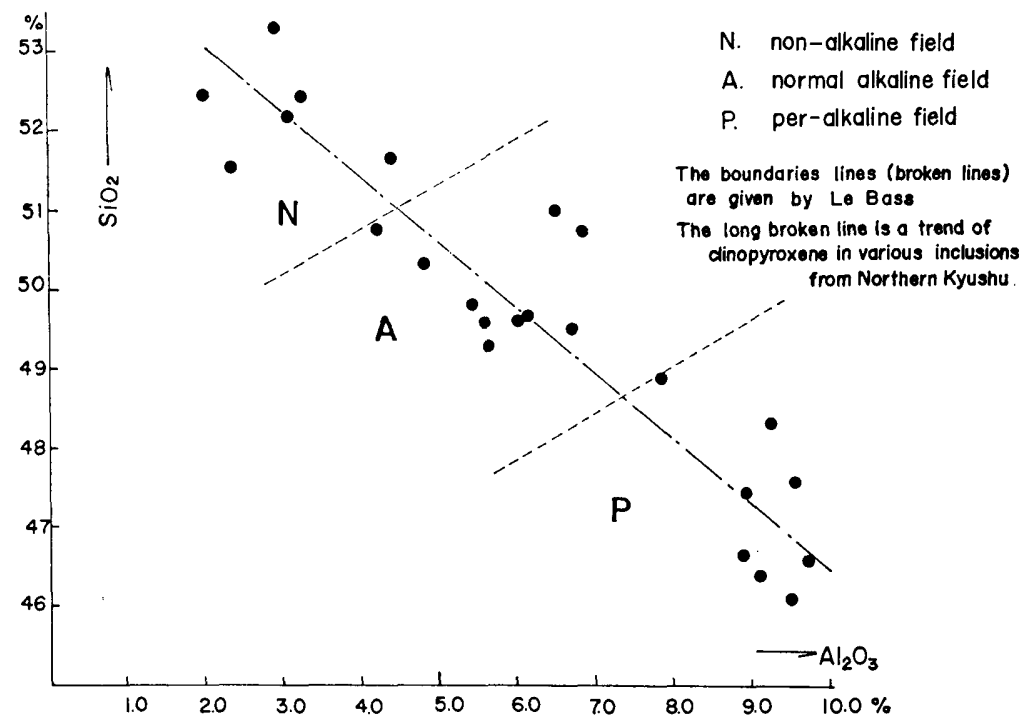

Fig. 7. The relation between $\mathrm{SiO}_{2}$ and $\mathrm{Al}_{2} \mathrm{O}_{3}$ in clinopyroxene.

The relation between $\mathrm{Al}_{2} \mathrm{O}_{3}$ and $\mathrm{SiO}_{2}$, and that between $\mathrm{Al}_{2} \mathrm{O}_{3}$ and $\mathrm{Cr}_{2} \mathrm{O}_{3}$ are shown in Figs. 7 and 8 respectively. As is clearly illustrated in Fig. 7, the $\mathrm{SiO}_{2}$ content in clinopyroxene increases with the decrease of $\mathrm{Al}_{2} \mathrm{O}_{3}$. The same tendency is also recognized in the relation between $\mathrm{Cr}_{2} \mathrm{O}_{3}$ and $\mathrm{Al}_{2} \mathrm{O}_{3}$. 
Table 10. Chemical composition of clinopyroxenes (anal. K. Ishibashi)

\begin{tabular}{|c|c|c|c|c|c|c|c|c|c|}
\hline No. & 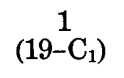 & $\stackrel{2}{\left(19-C_{2}\right)}$ & $\stackrel{3}{\left(19-C_{3}\right)}$ & $\left(19^{4}-C_{4}\right)$ & $\left(15^{5}-\mathrm{C}\right)$ & $\stackrel{6}{6}\left(19-C_{5}\right)$ & $\left(18^{7}-C_{1}\right)$ & $\stackrel{8}{\left(6-C_{1}\right)}$ & $\stackrel{9}{(14-C)}$ \\
\hline $\mathrm{SiO}_{2}$ & 46.05 & 46.36 & 46.54 & 46.67 & 47.23 & 47.37 & 47.55 & 48.29 & 48.88 \\
\hline $\mathrm{TiO}_{2}$ & 1.41 & 1.04 & 1.05 & 1.37 & 1.34 & 1.55 & 0.92 & 1.32 & 1.01 \\
\hline $\mathrm{Al}_{2} \mathrm{O}_{3}$ & 9.50 & 9.07 & 9.66 & 8.83 & 8.87 & 8.87 & 9.54 & 9.23 & 7.88 \\
\hline $\mathrm{Cr}_{2} \mathrm{O}_{3}$ & 0.20 & 0.05 & 0.10 & 0.09 & 0.04 & 0.14 & 0.19 & nd. & 0.12 \\
\hline $\mathrm{Fe}_{2} \mathrm{O}_{3}$ & 2.66 & 3.18 & 2.19 & 2.83 & 2.01 & 2.07 & 2.92 & 2.92 & 1.62 \\
\hline $\mathrm{FeO}$ & 6.75 & 6.11 & 6.87 & 6.41 & 6.35 & 6.21 & 3.59 & 3.92 & 5.21 \\
\hline $\mathrm{NiO}$ & 0.01 & 0.03 & 0.04 & 0.03 & 0.01 & 0.01 & nd. & nd. & 0.04 \\
\hline $\mathrm{MnO}$ & 0.16 & 0.18 & 0.12 & 0.10 & 0.09 & 0.13 & 0.14 & 0.14 & 0.16 \\
\hline $\mathrm{MgO}$ & 14.40 & 15.02 & 14.78 & 14.96 & 14.56 & 14.17 & 14.83 & 15.81 & 15.19 \\
\hline $\mathrm{CaO}$ & 18.41 & 18.03 & 18.05 & 18.04 & 18.31 & 18.26 & 19.27 & 17.28 & 18.57 \\
\hline $\mathrm{Na}_{2} \mathrm{O}$ & 0.85 & 0.91 & 0.93 & 0.85 & 0.78 & 0.96 & 0.97 & 1.27 & 0.87 \\
\hline $\mathrm{K}_{2} \mathrm{O}$ & 0.02 & 0.01 & 0.01 & 0.01 & 0.03 & 0.01 & 0.01 & 0.07 & 0.01 \\
\hline $\mathrm{P}_{2} \mathrm{O}_{5}$ & 0.01 & 0.00 & 0.01 & 0.01 & 0.01 & 0.01 & nd. & 0.01 & 0.00 \\
\hline $\mathrm{H}_{2} \mathrm{O}^{+}$ & 0.02 & 0.05 & 0.10 & 0.04 & 0.21 & 0.16 & nd. & nd. & 0.19 \\
\hline $\mathrm{H}_{2} \mathrm{O}^{-}$ & 0.04 & 0.01 & 0.04 & 0.03 & 0.24 & 0.12 & nd. & nd. & 0.08 \\
\hline Total & 100.49 & 100.05 & 100.49 & 100.27 & 100.08 & 100.06 & 99.93 & 100.26 & 99.83 \\
\hline $\mathrm{Si}$ & 1.703 & 1.708 & 1.716 & 1.724 & 1.809 & 1.750 & 1.745 & 1.758 & 1.795 \\
\hline $\mathrm{Al}$ & 0.297 & 0.292 & 0.284 & 0.276 & 0.280 & 0.250 & 0.255 & 0.242 & 0.205 \\
\hline $\mathrm{Cr}$ & - & - & - & - & - & - & - & - & - \\
\hline $\mathrm{Al}$ & 0.116 & 0.101 & 0.136 & 0.105 & 0.089 & 0.136 & 0.154 & 0.151 & 0.139 \\
\hline $\mathrm{Cr}$ & 0.004 & 0.002 & 0.004 & 0.004 & - & 0.004 & 0.004 & - & 0.004 \\
\hline $\mathrm{Fe}^{+3}$ & 0.075 & 0.088 & 0.062 & 0.080 & 0.060 & 0.058 & 0.079 & 0.079 & 0.044 \\
\hline $\mathrm{Ti}$ & 0.040 & 0.029 & 0.031 & 0.040 & 0.037 & 0.044 & 0.024 & 0.035 & 0.029 \\
\hline $\mathrm{Fe}$ & 0.208 & 0.188 & 0.212 & 0.197 & 0.202 & 0.191 & 0.110 & 0.118 & 0.159 \\
\hline $\mathrm{Ni}$ & - & - & - & - & - & - & - & - & - \\
\hline $\mathrm{Mn}$ & 0.007 & 0.007 & 0.002 & 0.002 & 0.002 & 0.002 & 0.002 & 0.002 & 0.007 \\
\hline $\mathrm{Mg}$ & 0.596 & 0.606 & 0.596 & 0.608 & 0.648 & 0.580 & 0.645 & 0.637 & 0.631 \\
\hline $\mathrm{Mg}$ & 0.204 & 0.225 & 0.220 & 0.221 & 0.188 & 0.204 & 0.171 & 0.225 & 0.201 \\
\hline $\mathrm{Ca}$ & 0.726 & 0.709 & 0.714 & 0.713 & 0.752 & 0.725 & 0.759 & 0.675 & 0.733 \\
\hline $\mathrm{Na}$ & 0.067 & 0.066 & 0.066 & 0.066 & 0.060 & 0.071 & 0.070 & 0.092 & 0.066 \\
\hline $\mathrm{K}$ & - & - & - & - & - & - & - & 0.004 & - \\
\hline $2-\mathrm{Si}$ & 0.297 & 0.292 & 0.284 & 0.276 & 0.280 & 0.250 & 0.255 & 0.242 & 0.205 \\
\hline $\mathrm{Al} / \mathrm{Cr}$ & 103 & 197 & 105 & 95.2 & large & 96.5 & 102 & - & 86.0 \\
\hline $\mathrm{Ca}$ & 40.1 & 38.9 & 39.5 & 39.1 & 40.6 & 41.2 & 43.0 & 39.0 & 41.3 \\
\hline $\mathrm{Mg}$ & 44.0 & 45.5 & 45.1 & 45.5 & 45.1 & 44.6 & 46.2 & 49.6 & 46.9 \\
\hline $\mathrm{Fe}$ & 15.9 & 15.5 & 15.4 & 15.4 & 14.3 & 14.2 & 10.8 & 11.4 & 11.8 \\
\hline
\end{tabular}


Table 10. continued

\begin{tabular}{|c|c|c|c|c|c|c|c|c|}
\hline No. & $\begin{array}{c}10 \\
(16-C)\end{array}$ & $\begin{array}{c}11 \\
\left(12-\mathrm{C}_{1}\right)\end{array}$ & $\begin{array}{c}12 \\
\left(12-\mathrm{C}_{2}\right)\end{array}$ & $\begin{array}{c}13 \\
(10-C)\end{array}$ & $\begin{array}{c}14 \\
(17-C)\end{array}$ & $\begin{array}{c}15 \\
(8-C)\end{array}$ & $\begin{array}{c}16 \\
(13-C)\end{array}$ & $\begin{array}{c}17 \\
\left(6-C_{2}\right)\end{array}$ \\
\hline $\mathrm{SiO}_{2}$ & 49.30 & 49.49 & 49.57 & 49.60 & 49.69 & 49.77 & 50.27 & 50.75 \\
\hline $\mathrm{TiO}_{2}$ & 0.61 & 0.50 & 0.32 & 0.26 & 0.50 & 0.33 & 0.61 & 0.67 \\
\hline $\mathrm{Al}_{2} \mathrm{O}_{3}$ & 5.61 & 6.69 & 5.57 & 6.04 & 6.14 & 5.47 & 4.83 & 6.86 \\
\hline $\mathrm{Cr}_{2} \mathrm{O}_{3}$ & 0.03 & 0.45 & 0.37 & 0.40 & 0.26 & 0.48 & 0.50 & nd. \\
\hline $\mathrm{Fe}_{2} \mathrm{O}_{3}$ & 1.98 & 2.37 & 2.71 & 1.79 & 2.46 & 2.27 & 1.62 & 1.45 \\
\hline $\mathrm{FeO}$ & 4.79 & 5.01 & 3.99 & 3.84 & 5.17 & 3.69 & 5.87 & 3.65 \\
\hline $\mathrm{NiO}$ & 0.01 & 0.08 & 0.10 & 0.03 & 0.07 & 0.05 & 0.08 & nd. \\
\hline $\mathrm{MnO}$ & 0.13 & 0.11 & 0.17 & 0.14 & 0.18 & 0.14 & 0.19 & 0.13 \\
\hline $\mathrm{MgO}$ & 15.11 & 18.70 & 20.01 & 16.06 & 15.70 & 17.08 & 17.54 & 17.35 \\
\hline $\mathrm{CaO}$ & 21.53 & 15.86 & 16.37 & 20.92 & 19.38 & 20.31 & 17.58 & 18.45 \\
\hline $\mathrm{Na}_{2} \mathrm{O}$ & 0.69 & 0.74 & 0.65 & 0.64 & 0.86 & 0.64 & 0.79 & 0.95 \\
\hline $\mathrm{K}_{2} \mathrm{O}$ & 0.02 & 0.04 & 0.03 & 0.02 & 0.03 & 0.02 & 0.05 & 0.07 \\
\hline $\mathrm{P}_{2} \mathrm{O}_{5}$ & 0.00 & 0.00 & 0.00 & 0.00 & 0.01 & 0.00 & 0.01 & 0.01 \\
\hline $\mathrm{H}_{2} \mathrm{O}^{+}$ & 0.12 & 0.12 & 0.13 & 0.11 & 0.02 & 0.04 & 0.06 & nd. \\
\hline $\mathrm{H}_{2} \mathrm{O}^{-}$ & 0.04 & 0.08 & 0.04 & 0.05 & 0.01 & 0.01 & 0.03 & nd. \\
\hline Total & 99.97 & 100.24 & 100.03 & 99.90 & 100.48 & 100.30 & 100.03 & 100.34 \\
\hline $\mathrm{Si}$ & 1.827 & 1.801 & 1.803 & 1.825 & 1.819 & 1.822 & 1.838 & 1.831 \\
\hline $\mathrm{Al}$ & 0.173 & 0.199 & 0.197 & 0.175 & 0.181 & 0.178 & 0.162 & 0.169 \\
\hline $\mathrm{Cr}$ & - & - & - & - & - & - & - & - \\
\hline $\mathrm{Al}$ & 0.071 & 0.087 & 0.043 & 0.075 & 0.083 & 0.059 & 0.044 & 0.125 \\
\hline $\mathrm{Cr}$ & - & 0.013 & 0.013 & 0.013 & 0.009 & 0.013 & 0.013 & - \\
\hline $\mathrm{Fe}^{+3}$ & 0.058 & 0.066 & 0.074 & 0.049 & 0.070 & 0.061 & 0.044 & 0.039 \\
\hline $\mathrm{Ti}$ & 0.018 & 0.013 & 0.009 & 0.007 & 0.013 & 0.009 & 0.018 & 0.019 \\
\hline $\mathrm{Fe}$ & 0.151 & 0.151 & 0.122 & 0.117 & 0.158 & 0.112 & 0.180 & 0.110 \\
\hline $\mathrm{Ni}$ & - & 0.002 & 0.002 & - & 0.002 & 0.002 & 0.002 & - \\
\hline $\mathrm{Mn}$ & 0.002 & 0.002 & 0.007 & 0.002 & 0.006 & 0.002 & 0.007 & 0.002 \\
\hline $\mathrm{Mg}$ & 0.736 & 0.690 & 0.754 & 0.730 & 0.686 & 0.776 & 0.713 & 0.726 \\
\hline $\mathrm{Mg}$ & 0.102 & 0.332 & 0.338 & 0.155 & 0.175 & 0.161 & 0.250 & 0.213 \\
\hline $\mathrm{Ca}$ & 0.849 & 0.620 & 0.640 & 0.823 & 0.760 & 0.795 & 0.689 & 0.714 \\
\hline $\mathrm{Na}$ & 0.049 & 0.048 & 0.022 & 0.022 & 0.065 & 0.044 & 0.057 & 0.069 \\
\hline $\mathrm{K}$ & - & - & - & - & - & - & 0.004 & 0.004 \\
\hline $2-\mathrm{Si}$ & 0.173 & 0.199 & 0.197 & 0.175 & 0.181 & 0.178 & 0.162 & 0.169 \\
\hline $\mathrm{Al} / \mathrm{Cr}$ & - & 22.0 & 18.4 & 19.2 & 29.3 & 18.2 & 15.8 & - \\
\hline $\mathrm{Ca}$ & 44.7 & 33.3 & 33.1 & 43.9 & 41.0 & 41.7 & 36.5 & 39.6 \\
\hline $\mathrm{Mg}$ & 44.1 & 54.9 & 56.4 & 47.1 & 46.5 & 49.1 & 51.2 & 52.0 \\
\hline $\mathrm{Fe}$ & 11.2 & 11.8 & 10.5 & 9.0 & 12.5 & 9.2 & 12.3 & 8.4 \\
\hline
\end{tabular}


Table 10. continued

\begin{tabular}{|c|c|c|c|c|c|c|c|c|}
\hline No. & $\begin{array}{c}18 \\
(7-C)\end{array}$ & $\begin{array}{c}19 \\
\left(18-C_{2}\right)\end{array}$ & $\begin{array}{c}20 \\
(9-C)\end{array}$ & $\begin{array}{c}21 \\
(2-\mathrm{C})\end{array}$ & $\begin{array}{c}22 \\
(11-C)\end{array}$ & $\begin{array}{c}23 \\
(4-C)\end{array}$ & $\begin{array}{c}24 \\
(5-C)\end{array}$ & $\stackrel{25}{(1-C)}$ \\
\hline $\mathrm{SiO}_{2}$ & 50.77 & 51.02 & 51.54 & 51.68 & 52.19 & 52.38 & 52.45 & 53.35 \\
\hline $\mathrm{TiO}_{2}$ & 0.21 & 0.67 & 0.18 & 0.47 & 0.09 & 0.08 & 0.10 & 0.08 \\
\hline $\mathrm{Al}_{2} \mathrm{O}_{3}$ & 4.19 & 6.52 & 2.31 & 4.44 & 3.09 & 3.23 & 2.01 & 2.95 \\
\hline $\mathrm{Cr}_{2} \mathrm{O}_{3}$ & 0.70 & 0.25 & 0.69 & 0.89 & 0.75 & 0.79 & 0.90 & 0.71 \\
\hline $\mathrm{Fe}_{2} \mathrm{O}_{3}$ & 1.99 & 1.79 & 1.65 & 1.43 & 0.78 & 0.75 & 0.83 & 0.84 \\
\hline $\mathrm{FeO}$ & 3.51 & 3.52 & 4.90 & 3.15 & 2.00 & 2.33 & 2.45 & 2.98 \\
\hline $\mathrm{NiO}$ & 0.01 & nd. & nd. & 0.07 & 0.03 & 0.06 & 0.03 & 0.03 \\
\hline $\mathrm{MnO}$ & 0.14 & 0.13 & 0.17 & 0.09 & 0.09 & 0.10 & 0.09 & 0.09 \\
\hline $\mathrm{MgO}$ & 17.21 & 17.93 & 16.95 & 16.68 & 18.57 & 18.07 & 18.01 & 18.42 \\
\hline $\mathrm{CaO}$ & 20.32 & 17.74 & 20.98 & 20.26 & 22.03 & 21.82 & 22.74 & 19.57 \\
\hline $\mathrm{Na}_{2} \mathrm{O}$ & 0.49 & 0.85 & 0.45 & 0.87 & 0.58 & 0.55 & 0.86 & 0.54 \\
\hline $\mathrm{K}_{2} \mathrm{O}$ & 0.02 & 0.05 & 0.03 & 0.02 & 0.02 & 0.02 & 0.02 & 0.01 \\
\hline $\mathrm{P}_{2} \mathrm{O}_{5}$ & 0.00 & nd. & 0.01 & 0.00 & 0.00 & 0.00 & 0.00 & 0.00 \\
\hline $\mathrm{H}_{2} \mathrm{O}^{+}$ & 0.06 & nd. & nd. & 0.03 & 0.03 & 0.03 & 0.03 & 0.16 \\
\hline $\mathrm{H}_{2} \mathrm{O}^{-}$ & 0.03 & nd. & nd. & 0.02 & 0.05 & 0.01 & 0.02 & 0.04 \\
\hline Total & 99.65 & 100.47 & 99.86 & 100.10 & 100.30 & 100.22 & 100.54 & 99.77 \\
\hline $\mathrm{Si}$ & 1.862 & 1.835 & 1.895 & 1.880 & 1.893 & 1.882 & 1.908 & 1.933 \\
\hline $\mathrm{Al}$ & 0.138 & 0.165 & 0.102 & 0.120 & 0.107 & 0.118 & 0.087 & 0.067 \\
\hline $\mathrm{Cr}$ & - & - & 0.003 & - & - & - & 0.005 & - \\
\hline $\mathrm{Al}$ & 0.042 & 0.111 & - & 0.068 & 0.024 & 0.016 & - & 0.059 \\
\hline $\mathrm{Cr}$ & 0.022 & 0.009 & 0.019 & 0.026 & 0.021 & 0.021 & 0.011 & 0.021 \\
\hline $\mathrm{Fe}^{+3}$ & 0.057 & 0.047 & 0.046 & 0.039 & 0.021 & 0.021 & 0.022 & 0.021 \\
\hline $\mathrm{Ti}$ & 0.006 & 0.019 & 0.007 & 0.013 & 0.002 & 0.002 & 0.002 & 0.002 \\
\hline $\mathrm{Fe}$ & 0.108 & 0.106 & 0.150 & 0.096 & 0.061 & 0.069 & 0.076 & 0.091 \\
\hline $\mathrm{Ni}$ & - & - & - & 0.002 & - & 0.002 & -- & - \\
\hline $\mathrm{Mn}$ & 0.002 & 0.002 & 0.007 & 0.002 & 0.002 & 0.002 & 0.002 & 0.002 \\
\hline $\mathrm{Mg}$ & 0.781 & 0.718 & 0.797 & 0.766 & 0.907 & 0.862 & 0.939 & 0.802 \\
\hline $\mathrm{Mg}$ & 0.168 & 0.249 & 0.138 & 0.144 & 0.103 & 0.116 & 0.051 & 0.200 \\
\hline $\mathrm{Ca}$ & 0.797 & 0.682 & 0.827 & 0.790 & 0.853 & 0.841 & 0.884 & 0.761 \\
\hline $\mathrm{Na}$ & 0.035 & 0.065 & 0.035 & 0.066 & 0.044 & 0.043 & 0.065 & 0.039 \\
\hline $\mathbf{K}$ & - & 0.004 & - & - & - & - & - & - \\
\hline $2-\mathrm{Si}$ & 0.138 & 0.165 & 0.105 & 0.120 & 0.107 & 0.118 & 0.092 & 0.067 \\
\hline $\mathrm{Al} / \mathrm{Cr}$ & 8.1 & 30.6 & 4.8 & 7.2 & 6.2 & 6.3 & 5.4 & 6.0 \\
\hline $\mathrm{Ca}$ & 41.7 & 37.8 & 42.2 & 43.0 & 43.8 & 44.0 & 44.7 & 40.5 \\
\hline $\mathrm{Mg}$ & 49.6 & 53.6 & 47.5 & 49.6 & 51.9 & 51.2 & 50.2 & 53.4 \\
\hline $\mathrm{Fe}$ & 8.7 & 8.6 & 10.3 & 7.4 & 4.3 & 4.8 & 5.1 & 6.1 \\
\hline
\end{tabular}




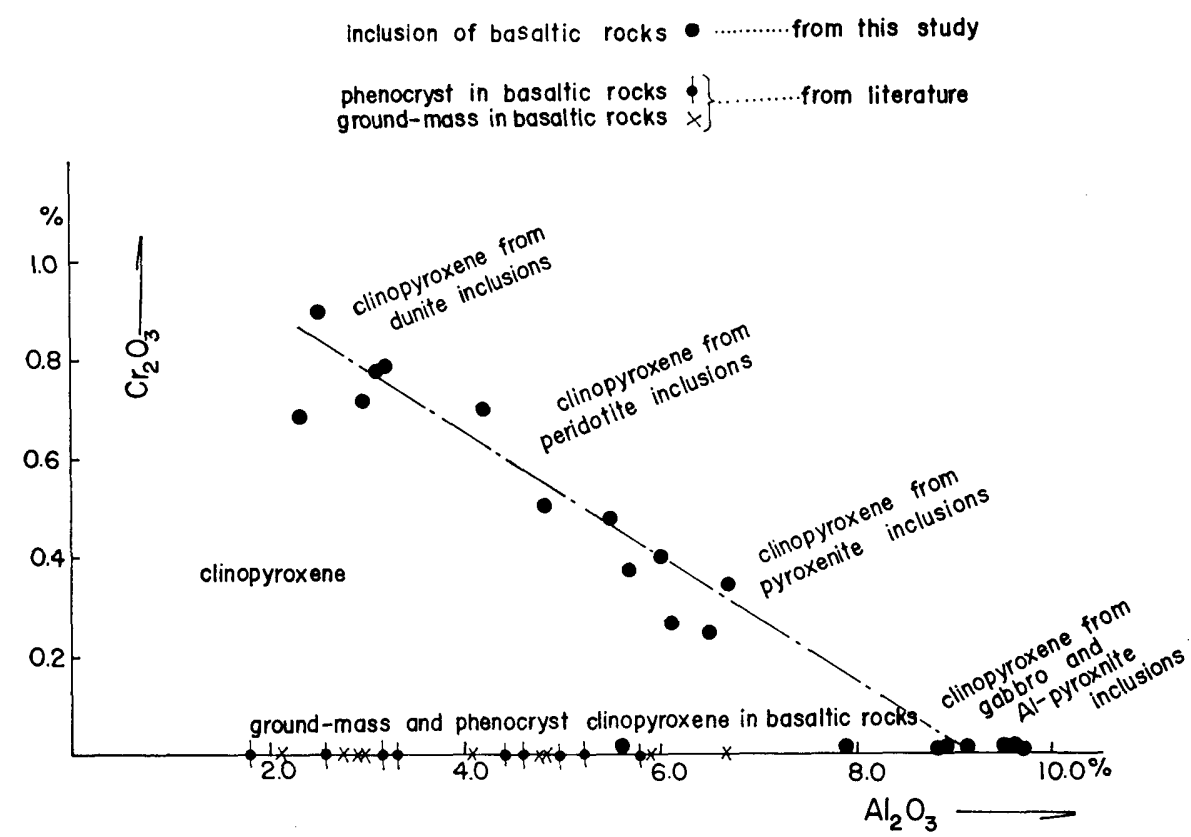

Fig. 8. The $\mathrm{Cr}_{2} \mathrm{O}_{3}-\mathrm{Al}_{2} \mathrm{O}_{3}$ relation of clinopyroxenes in various kinds of rocks.

In calculation of pyroxene formula, the deficiency of $\mathrm{Si}$ in the $\mathrm{Z}$ position is filled at first with $\mathrm{Al}$, and then, if necessary, with $\mathrm{Cr}$, until the $\mathrm{Z}$ position becomes 2.000. In the present study $\mathrm{Fe}$ and $\mathrm{Ti}$ were not entered into $\mathrm{Z}$ position. Calculation of various kinds of pyroxene molecules from chemical composition has been devised by Kushiro (1962) and YODER and TilleY (1962). A slight modification has been made by ONUKI (1965) in the case when the $\mathrm{Cr}_{2} \mathrm{O}_{3}$ content in clinopyroxene is known. He has proposed $\mathrm{NaCrSi}_{2} \mathrm{O}_{6}$ and $\mathrm{CaCrAlSiO}_{6}$ molecules, in addition to pyroxene molecules which have been proposed by KusHIRo (1962). In this study, various pyroxene molecules are calculated in accordance with the method of KUSHIRo (1962) and of ONUKI (1965), with the results shown in Table 11. As is clear in this table, the $\mathrm{CaAl}_{2} \mathrm{SiO}_{6}$ molecules of clinopyroxene are richest in phenocryst of basalt, and the content decreases from gabbro and pyroxenite through peridotite and to dunite. The variation in composition $(\mathrm{Ca}$, $\mathrm{Mg}$ and $\mathrm{Fe}$ ) of clinopyroxene is shown in Fig. 15.

2) The relation between 2-Si and $\mathrm{Al} / \mathrm{Cr}$ in clinopyroxene

It has long been known that clinopyroxene solid solution contains a certain amount of $\mathrm{Cr}_{2} \mathrm{O}_{3}$ and $\mathrm{Al}_{2} \mathrm{O}_{3}$. Extensive studies have been made about the $\mathrm{Al}_{2} \mathrm{O}_{3}$ content in clinopyroxene by KUSHIRo $(1960,1962)$, Le BASS (1962), KUNO (1964), YAMAGUCHI (1964) and ONUKI $(1965,1966)$, while the $\mathrm{Cr}_{2} \mathrm{O}_{3}$ content in clinopyroxene has not been sufficiently discussed. Figure 9 shows 2-Si against $\mathrm{Al} / \mathrm{Cr}$ of clinopyroxene in basic and ultrabasic inclusions from northern Kyushu. It is interesting to note in Fig. 9 that clinopyroxenes in olivine-rich inclusions from northern Kyushu are distinguished into two distinct dfferent types. One is characterized by small $\mathrm{Al} / \mathrm{Cr}$ and $2-\mathrm{Si}$, and the other is by large $\mathrm{Al} / \mathrm{Cr}$ and $2-\mathrm{Si}$, on the basis of six oxygen atoms. The former is contained in dunite, Cr-diopside 
Table 11. Proportion of pyroxene molecules

\begin{tabular}{|c|c|c|c|c|c|c|c|c|c|c|}
\hline No. & $\mathrm{NaCrSi}_{2} \mathrm{O}_{6}$ & $\mathrm{NaFeSi}{ }_{2} \mathrm{O}_{6}$ & $\mathrm{NaAlSi}{ }_{2} \mathrm{O}_{6}$ & $\mathrm{CaTiAl}_{2} \mathrm{O}_{6}$ & $\left|\mathrm{CaFe}^{+3} \mathrm{AlSiO}_{6}\right|$ & $\mathrm{CaCrAlSiO}_{6}$ & $\mathrm{CaAl}_{2} \mathrm{SiO}_{6}$ & $\mathrm{Ca}_{2} \mathrm{Si}_{2} \mathrm{O}_{6}$ & $\mathrm{Mg}_{2} \mathrm{Si}_{2} \mathrm{O}_{6}$ & $\mathrm{Fe}_{2} \mathrm{Si}_{2} \mathrm{O}_{6}$ \\
\hline 1 & 0.4 & 6.3 & - & 4.0 & 1.2 & - & 16.0 & 20.7 & 40.0 & 10.7 \\
\hline 2 & 0.2 & 4.4 & - & 2.9 & 2.4 & - & 15.6 & 25.5 & 41.6 & 9.7 \\
\hline 3 & 0.4 & 6.2 & - & 3.1 & - & - & 17.4 & 25.5 & 40.8 & 10.7 \\
\hline 4 & 0.4 & 6.2 & - & 4.0 & 1.8 & - & 14.1 & 25.7 & 41.5 & 9.4 \\
\hline 5 & - & 6.0 & 一 & 3.7 & - & - & 14.8 & 25.9 & 41.8 & 10.2 \\
\hline 6 & 0.4 & 6.6 & - & 4.4 & 1.3 & - & 14.0 & 26.0 & 33.9 & 9.5 \\
\hline 8 & - & 7.9 & - & 3.5 & - & - & 15.3 & 23.8 & 43.1 & 6.0 \\
\hline 9 & 0.4 & 4.4 & 1.8 & 2.9 & - & - & 13.2 & 28.6 & 41.6 & 8.3 \\
\hline 10 & - & 4.9 & - & 1.8 & 0.9 & - & 9.9 & 36.2 & 41.9 & 7.1 \\
\hline 11 & 1.3 & 3.5 & - & 1.3 & 3.1 & - & 11.4 & 23.1 & 51.1 & 7.6 \\
\hline 12 & 1.3 & 0.5 & - & 0.9 & 5.2 & - & 7.8 & 24.4 & 54.7 & 6.5 \\
\hline 13 & 1.3 & 0.5 & - & 0.7 & 4.0 & - & 9.8 & 33.9 & 44.2 & 5.9 \\
\hline 14 & 0.9 & 5.4 & - & 1.3 & 1.4 & - & 11.2 & 31.1 & 43.0 & 8.2 \\
\hline 16 & 1.3 & 4.4 & 0.4 & 1.8 & - & - & 8.3 & 29.4 & 48.2 & 9.3 \\
\hline 17 & - & 3.9 & 3.4 & 1.9 & - & - & 11.1 & 29.2 & 46.9 & 5.6 \\
\hline 18 & 2.2 & 1.3 & - & 0.6 & 4.4 & - & 6.4 & 34.1 & 47.4 & 5.5 \\
\hline 19 & 0.9 & 4.7 & 1.3 & 1.9 & - & - & 10.6 & 27.2 & 48.3 & 5.4 \\
\hline 20 & 2.2 & 1.3 & - & 0.7 & 3.3 & - & 2.7 & 38.0 & 41.7 & 7.8 \\
\hline 21 & 2.6 & 3.9 & 0.1 & 1.3 & - & - & 8.0 & 34.8 & 45.6 & 4.9 \\
\hline 22 & 2.1 & 2.1 & 0.2 & 0.2 & - & - & 6.2 & 39.4 & 50.5 & 3.2 \\
\hline 23 & 2.1 & 2.1 & 0.1 & 0.2 & - & - & 6.4 & 38.7 & 48.9 & 3.2 \\
\hline 24 & 1.6 & 2.2 & 2.7 & 0.2 & - & - & 2.8 & 42.7 & 44.6 & 3.9 \\
\hline 25 & 2.1 & 1.5 & - & 0.2 & 0.3 & - & 5.9 & 32.6 & 50.1 & 4.6 \\
\hline
\end{tabular}

The number is the same as in Table 10 . 


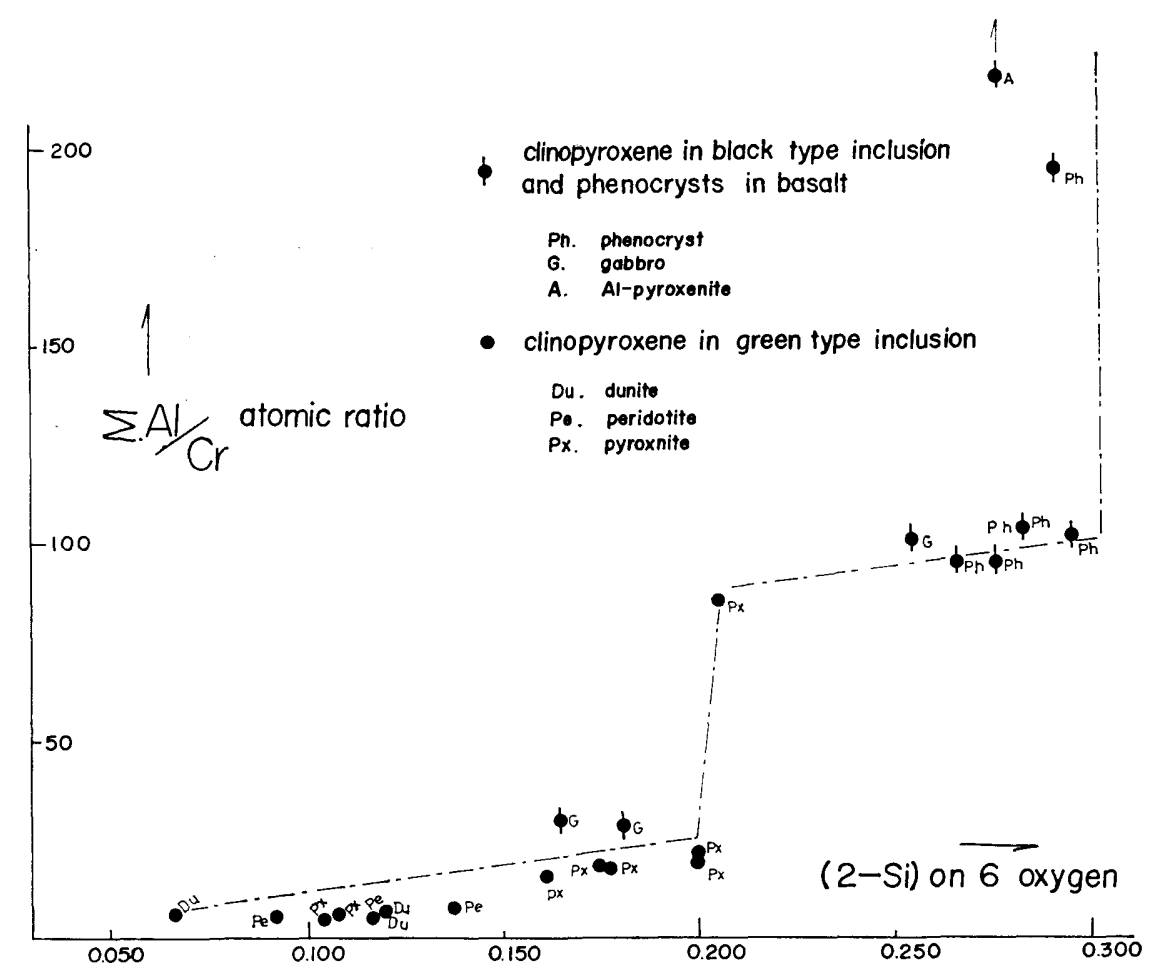

Fig. 9. $\mathrm{Al} / \mathrm{Cr}$ and 2-Si relation in clinopyroxenes of basic and ultrabasic inclusions from Karatsu area.

peridotite and Cr-pyroxenite (green type inclusion), and the latter is in Alpyroxenite, gabbros (black type inclusion) and phenocrystic clinopyroxene in basaltic host rocks. The $2-\mathrm{Si}$ and $\mathrm{Al} / \mathrm{Cr}$ relation of the former is almost linear from dunite to pyroxenite, and no compositional gap exists in it.

KUSHIRo (1960) has suggested that clinopyroxene in basaltic rocks reflects distinct character of bulk chemical composition of their host magma. He has demonstrated that clinopyroxene of alkali olivine basalt is richer in $\mathrm{Al}_{2} \mathrm{O}_{3}$ and poorer in $\mathrm{SiO}_{2}$ than that of tholeiitic basalt, and the clinopyroxene of high aluminou basalt is of intermediate composition between that of alkali and toleiitic basalts.

As shown in Fig. 9, the clinopyroxene in olivine-rich inclusions seems to show an inverse relation. Namely, the content of $\mathrm{SiO}_{2}$ in clinopyroxene is highest in dunite, decreasing from dunite through $\mathrm{Cr}$-diopside peridotite to $\mathrm{Cr}$-pyroxenite, and the $\mathrm{Al}_{2} \mathrm{O}_{3}$ content increases with decreasing $\mathrm{SiO}_{2}$, whereas the bulk $\mathrm{SiO}_{2}$ content is lowest in dunite and gradually increases from dunite to Cr-pyroxenite. On the other hand, clinopyroxenes of Al-pyroxenite and gabbros are always richer in $\mathrm{Al}_{2} \mathrm{O}_{3}$ and poorer in $\mathrm{SiO}_{2}$ than that of green type inclusions. There is a distinct difference between clinopyroxene of olivine-rich inclusions and that of phenocrysts and groundmass of basalts as mentioned by KusHiro (1960). The $\mathrm{SiO}_{2}$ content in clinopyroxene of green type inclusions decreases with increasing bulk $\mathrm{SiO}_{2}$ content of the inclusions. If these olivine-rich inclusions in basaltic 
rocks were formed by direct crystallization from basaltic magma, the chemistry should reflect the $\mathrm{SiO}_{2}, \mathrm{Al}_{2} \mathrm{O}_{3}$ and $\mathrm{Cr}_{2} \mathrm{O}_{3}$ contents of the constituent minerals in a reasonable way.

If we follow the suggestion of KusHiRo (1960) as mentioned early, the clinopyroxenes in the inclusions should be poorer in $\mathrm{SiO}_{2}$ than that of clinopyroxenes from tholeiitic basalts. However, the situation is reversed in the present study (Fig. 7).

Many excellent analyses of clinopyroxenes from various kinds of rocks have been reported. A few data, however, are available with respect to $\mathrm{Cr}_{2} \mathrm{O}_{3}$ content.

Valuable information about $\mathrm{Cr}_{2} \mathrm{O}_{3}$ contents of clinopyroxenes of olivine-rich

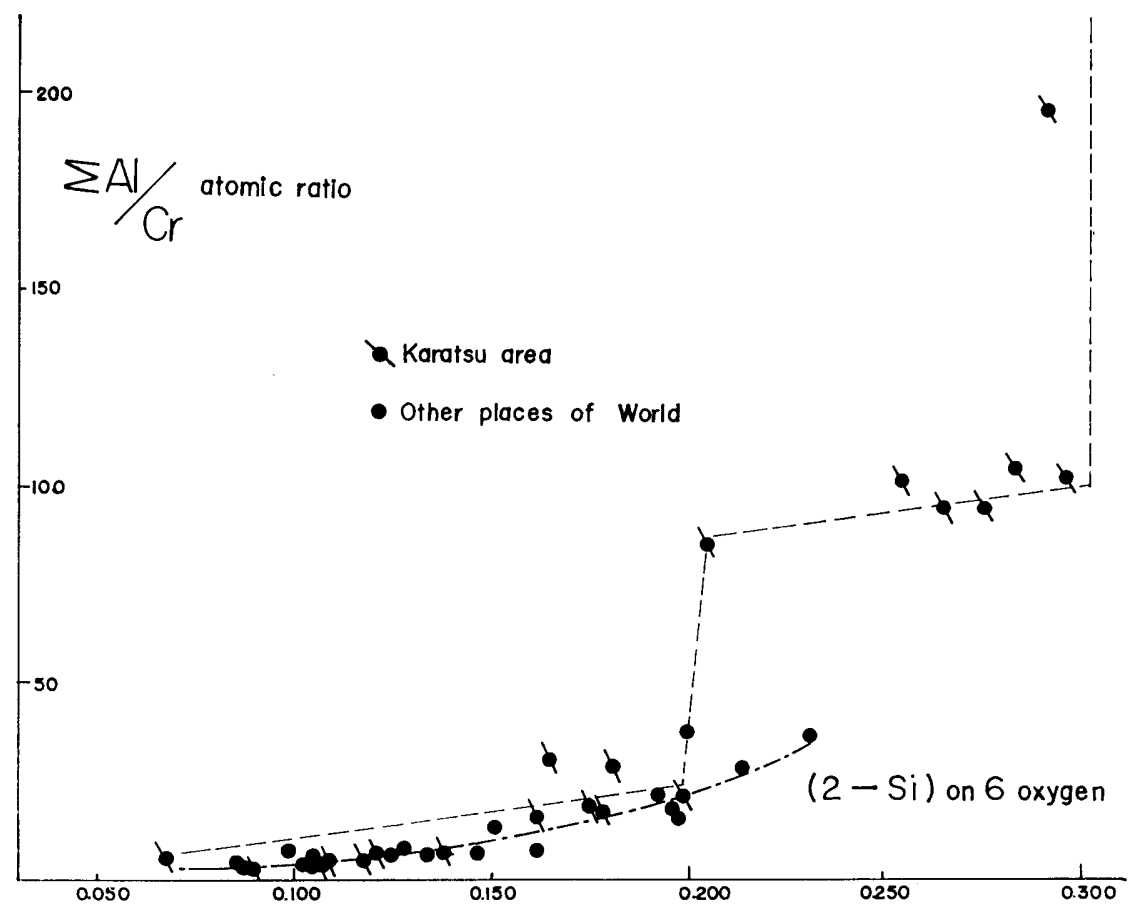

Fig. 10. Diagram showing the relation between $\mathrm{Al}-\mathrm{Cr}$ and 2-Si in clinopyroxenes from Katatsu area and some other places of the world.

inclusions in basaltic rocks were given by Ross et al. (1954), WILSHIRE and BINNS (1961) and KUNo (1964). Previous chemical data of clinopyroxenes from olivine-rich inclusions in basaltic rocks are assembled in Table 12 and the relation between 2-Si and $\mathrm{Al} / \mathrm{Cr}$ is shown in Fig. 10. It is noted that the clinopyroxenes of olivine-rich inclusions from other localities exhibit exactly the same linear relation between $2-\mathrm{Si}$ and $\mathrm{Al} / \mathrm{Cr}$ with those of dunite, Cr-diopside peridotite and Cr-pyroxenite from northern Kyushu. On the other hand, the analytical data on the clinopyroxenes of Al-pyroxenite and gabbro (the black type inclusion) from the same area deviate much from this general linear relation. Why does this difference exist between the clinopyroxenes of the green type inclusion and that of the black type inclusion? 
Table 12. Chemical composition of clinopyroxene of olivine-rich inclusions in basaltic rocks

\begin{tabular}{|c|c|c|c|c|c|c|c|c|c|}
\hline No. & 1 & 2 & 3 & 4 & 5 & 6 & 7 & 8 & 9 \\
\hline $\mathrm{SiO}_{2}$ & 51.43 & 50.66 & 52.43 & 49.35 & 52.74 & 54.17 & 50.94 & 51.91 & 48.64 \\
\hline $\mathrm{TiO}_{2}$ & 0.32 & 0.68 & 0.37 & 0.34 & 0.19 & 0.02 & 0.46 & 0.26 & 2.13 \\
\hline $\mathrm{Al}_{2} \mathrm{O}_{3}$ & 4.46 & 5.13 & 4.16 & 4.92 & 4.86 & 1.43 & 5.78 & 4.77 & 5.24 \\
\hline $\mathrm{Cr}_{2} \mathrm{O}_{3}$ & 0.98 & 0.83 & 1.06 & 1.06 & 0.66 & 1.24 & 0.59 & 0.80 & 0.05 \\
\hline $\mathrm{Fe}_{2} \mathrm{O}_{3}$ & 0.83 & 0.85 & 2.66 & 0.77 & 1.42 & 1.43 & 1.39 & 0.92 & 4.00 \\
\hline $\mathrm{FeO}$ & 2.53 & 2.94 & 3.53 & 2.19 & 1.08 & 1.09 & 2.77 & 2.39 & 5.20 \\
\hline $\mathrm{MnO}$ & 0.09 & 0.10 & 0.12 & 0.07 & 0.09 & 0.06 & 0.10 & 0.07 & 0.25 \\
\hline $\mathrm{NiO}$ & - & - & 0.08 & 0.05 & 0.051 & - & 0.03 & 0.04 & 0.06 \\
\hline $\mathrm{MgO}$ & 16.41 & 16.28 & 18.19 & 19.86 & 16.76 & 17.37 & 17.47 & 16.02 & 12.26 \\
\hline $\mathrm{CaO}$ & 21.61 & 21.38 & 16.56 & 19.75 & 20.47 & 22.63 & 19.43 & 21.75 & 21.01 \\
\hline $\mathrm{Na}_{2} \mathrm{O}$ & 0.56 & 0.49 & 0.96 & 1.23 & 1.41 & nd. & 0.72 & 0.74 & 1.21 \\
\hline $\mathrm{K}_{2} \mathrm{O}$ & 0.08 & 0.08 & 0.07 & nil. & 0.02 & nd. & 0.03 & 0.04 & 0.14 \\
\hline $\mathrm{P}_{2} \mathrm{O}_{5}$ & - & - & - & nd. & - & - & - & - & nil. \\
\hline $\mathrm{H}_{2} \mathrm{O}^{+}$ & 0.20 & 0.15 & 0.09 & 0.17 & nd. & 0.23 & 0.02 & 0.09 & 0.04 \\
\hline $\mathrm{H}_{2} \mathrm{O}^{-}$ & 0.05 & 0.20 & 0.11 & 0.08 & nd. & nd. & 0.07 & 0.11 & 0.06 \\
\hline Total & 99.55 & 99.77 & 100.33 & 99.84 & 99.75 & 99.67 & 99.80 & 99.91 & 100.29 \\
\hline $\mathrm{Si}$ & 1.883 & 1.857 & 1.895 & 1.795 & 1.906 & 1.969 & 1.858 & 1.894 & 1.813 \\
\hline $\mathrm{Al}$ & 0.117 & 0.143 & 0.105 & 0.205 & 0.094 & 0.031 & 0.142 & 0.106 & 0.187 \\
\hline $\mathrm{Ti}$ & 0.009 & 0.019 & 0.010 & 0.009 & 0.005 & 0.001 & 0.013 & 0.007 & 0.060 \\
\hline $\mathrm{Al}$ & 0.075 & 0.078 & 0.072 & 0.006 & 0.113 & 0.030 & 0.107 & 0.099 & 0.043 \\
\hline $\mathrm{Fe}^{+3}$ & 0.023 & 0.023 & 0.072 & 0.021 & 0.039 & 0.039 & 0.038 & 0.025 & 0.112 \\
\hline $\mathrm{Cr}$ & 0.028 & 0.024 & 0.030 & 0.030 & 0.019 & 0.036 & 0.017 & 0.023 & 0.001 \\
\hline $\mathrm{Fe}$ & 0.077 & 0.090 & 0.107 & 0.066 & 0.033 & 0.033 & 0.084 & 0.073 & 0.162 \\
\hline $\mathrm{Mn}$ & 0.003 & 0.003 & 0.004 & 0.002 & 0.003 & 0.002 & 0.003 & 0.002 & 0.008 \\
\hline $\mathrm{Ni}$ & - & - & 0.002 & 0.001 & 0.001 & - & 0.001 & 0.001 & 0.002 \\
\hline $\mathrm{Mg}$ & 0.901 & 0.895 & 0.980 & 1.083 & 0.903 & 0.941 & 0.949 & 0.871 & 0.681 \\
\hline $\mathrm{Ca}$ & 0.848 & 0.840 & 0.641 & 0.770 & 0.793 & 0.881 & 0.739 & 0.844 & 0.839 \\
\hline $\mathrm{Na}$ & 0.039 & 0.035 & 0.067 & 0.086 & 0.099 & - & 0.051 & 0.052 & 0.087 \\
\hline $\mathrm{K}$ & 0.004 & 0.004 & 0.003 & - & - & - & 0.001 & 0.002 & 0.007 \\
\hline $2-\mathrm{Si}$ & 0.117 & 0.143 & 0.105 & 0.205 & 0.094 & 0.031 & 0.142 & 0.106 & 0.187 \\
\hline $\mathrm{Al} / \mathrm{Cr}$ & 6.8 & 9.2 & 5.9 & 7.0 & 5.3 & 1.7 & 14.6 & 8.9 & 247 \\
\hline
\end{tabular}

No. 1, 2 ONUKI, H. (1965)

No. 3 ERNST, T. (1936)

No. 4 HAMAD, S. D. (1962)

No. 5, 6 Ohara, M. J. and Mercy, L. P. (1966) 
Table 12. continued

\begin{tabular}{|c|c|c|c|c|c|c|c|c|}
\hline No. & 10 & 11 & 12 & 13 & 14 & 15 & 16 & 17 \\
\hline $\mathrm{SiO}_{2}$ & 46.41 & 56.38 & 47.23 & 47.67 & 50.67 & 52.30 & 50.63 & 51.78 \\
\hline $\mathrm{TiO}_{2}$ & 2.11 & 0.22 & 1.37 & 1.14 & 0.56 & 0.10 & 0.40 & 0.38 \\
\hline $\mathrm{Al}_{2} \mathrm{O}_{3}$ & 7.80 & 13.74 & 7.45 & 5.10 & 4.11 & 2.52 & 4.70 & 5.14 \\
\hline $\mathrm{Cr}_{2} \mathrm{O}_{3}$ & 0.02 & 0.001 & 0.012 & 0.35 & 0.62 & 0.41 & 0.26 & 0.95 \\
\hline $\mathrm{Fe}_{2} \mathrm{O}_{3}$ & 5.60 & 4.66 & 3.01 & 3.14 & 1.94 & 1.61 & 1.68 & 1.75 \\
\hline $\mathrm{FeO}$ & 4.70 & 1.24 & 7.18 & 4.38 & 3.17 & 3.03 & 3.80 & 2.14 \\
\hline $\mathrm{MnO}$ & 0.31 & 0.11 & 0.20 & 0.09 & nd. & 0.12 & 0.15 & 0.12 \\
\hline $\mathrm{NiO}$ & 0.02 & 0.022 & nd. & nd. & nd. & nd. & - & 0.041 \\
\hline $\mathrm{MgO}$ & 11.51 & 6.70 & 13.66 & 16.35 & 17.13 & 17.98 & 16.35 & 16.04 \\
\hline $\mathrm{CaO}$ & 20.05 & 7.73 & 18.90 & 19.15 & 20.43 & 21.83 & 21.04 & 20.32 \\
\hline $\mathrm{Na}_{2} \mathrm{O}$ & 1.22 & 9.01 & 0.93 & 0.57 & 0.38 & 0.27 & 0.48 & 1.06 \\
\hline $\mathrm{K}_{2} \mathrm{O}$ & 0.14 & 0.06 & 0.10 & 0.25 & 0.25 & 0.10 & 0.05 & 0.08 \\
\hline $\mathrm{P}_{2} \mathrm{O}_{5}$ & nil. & nd. & tr. & nd. & nd. & 0.06 & 0.01 & 0.04 \\
\hline $\mathrm{H}_{2} \mathrm{O}^{+}$ & 0.09 & nd. & 0.49 & nd. & nd. & 0.36 & 0.55 & - \\
\hline $\mathrm{H}_{2} \mathrm{O}^{-}$ & 0.06 & nd. & tr. & 0.12 & 0.06 & 0.05 & 0.10 & - \\
\hline Total & 100.05 & 99.87 & 100.43 & 100.29 & 99.32 & 100.64 & 100.20 & 99.84 \\
\hline $\mathrm{Si}$ & 1.738 & 1.981 & 1.762 & 1.830 & 1.865 & 1.906 & 1.863 & 1.881 \\
\hline $\mathrm{Al}$ & 0.262 & 0.019 & 0.238 & 0.170 & 0.135 & 0.094 & 0.137 & 0.119 \\
\hline $\mathrm{Ti}$ & 0.059 & 0.006 & 0.039 & 0.061 & 0.015 & 0.003 & 0.011 & 0.011 \\
\hline $\mathrm{Al}$ & 0.082 & 0.550 & 0.089 & 0.051 & 0.037 & 0.014 & 0.067 & 0.089 \\
\hline $\mathrm{Fe}^{+3}$ & 0.158 & 0.123 & 0.085 & 0.087 & 0.057 & 0.044 & 0.046 & 0.048 \\
\hline $\mathrm{Cr}$ & 0.000 & 0.000 & 0.000 & 0.011 & 0.019 & 0.012 & 0.008 & 0.031 \\
\hline $\mathrm{Fe}$ & 0.162 & 0.036 & 0.224 & 0.135 & 0.097 & 0.092 & 0.117 & 0.063 \\
\hline $\mathrm{Mn}$ & 0.010 & 0.003 & 0.006 & 0.003 & 0.000 & 0.004 & 0.005 & 0.002 \\
\hline $\mathrm{Ni}$ & 0.001 & 0.001 & - & - & - & - & - & - \\
\hline $\mathrm{Mg}$ & 0.642 & 0.351 & 0.759 & 0.898 & 0.938 & 0.976 & 0.896 & 0.874 \\
\hline $\mathrm{Ca}$ & 0.804 & 0.291 & 0.755 & 0.756 & 0.801 & 0.852 & 0.829 & 0.789 \\
\hline $\mathrm{Na}$ & 0.089 & 0.614 & 0.064 & 0.041 & 0.027 & 0.019 & 0.034 & 0.078 \\
\hline $\mathrm{K}$ & 0.007 & 0.000 & 0.004 & 0.011 & 0.011 & 0.004 & 0.002 & 0.004 \\
\hline 2-Si & 0.262 & 0.019 & 0.238 & 0.170 & 0.135 & 0.094 & 0.137 & 0.119 \\
\hline $\mathrm{Al} / \mathrm{Cr}$ & large & large & large & 20.0 & 9.1 & 9.0 & 25.5 & 7.1 \\
\hline
\end{tabular}

No. 7-10 Wilshire, H. G. and Binns, R. A. (1961)

No. 11 Ohara, M. J. and Mercy, L. P. (1966)

No. 12 KUno, H. (1964)

No. 13, 14 MiYake, T. (1948)

No. 15, 16 Kuno, H. (1964) 
Table 12. continued

\begin{tabular}{|c|c|c|c|c|c|c|c|c|}
\hline No. & 18 & 19 & 20 & 21 & 22 & 23 & 24 & 25 \\
\hline $\mathrm{SiO}_{2}$ & 51.42 & 50.36 & 51.17 & 52.48 & 51.41 & 52.12 & 51.87 & 50.24 \\
\hline $\mathrm{TiO}_{2}$ & 0.24 & 0.79 & 0.28 & 0.62 & 0.33 & 0.80 & 0.65 & 0.72 \\
\hline $\mathrm{Al}_{2} \mathrm{O}_{3}$ & 5.97 & 6.58 & 6.46 & 3.56 & 4.69 & 4.08 & 1.72 & 4.57 \\
\hline $\mathrm{Cr}_{2} \mathrm{O}_{3}$ & 0.80 & 0.92 & 1.20 & 2.12 & 1.03 & 1.44 & 1.07 & 1.22 \\
\hline $\mathrm{Fe}_{2} \mathrm{O}_{3}$ & 1.43 & 1.53 & 0.85 & 1.13 & 0.69 & 0.82 & 1.61 & 2.53 \\
\hline $\mathrm{FeO}$ & 2.57 & 2.66 & 2.87 & 2.52 & 2.64 & 2.24 & 3.66 & 4.04 \\
\hline $\mathrm{MnO}$ & 0.15 & 0.09 & 0.16 & 0.15 & 0.11 & 0.09 & 0.06 & 0.13 \\
\hline $\mathrm{NiO}$ & 0.045 & 0.037 & 0.036 & 0.034 & 0.040 & 0.041 & 0.040 & - \\
\hline $\mathrm{MgO}$ & 16.17 & 15.59 & 17.65 & 17.03 & 16.32 & 16.48 & 17.94 & 16.53 \\
\hline $\mathrm{CaO}$ & 19.95 & 18.78 & 18.27 & 19.00 & 21.63 & 19.34 & 20.92 & 19.96 \\
\hline $\mathrm{Na}_{2} \mathrm{O}$ & 1.36 & 1.41 & 1.20 & - & 0.72 & 2.14 & 0.33 & 0.58 \\
\hline $\mathrm{K}_{2} \mathrm{O}$ & 0.10 & 0.08 & 0.08 & - & 0.04 & 0.08 & 0.02 & 0.04 \\
\hline $\mathrm{P}_{2} \mathrm{O}_{5}$ & 0.03 & 0.03 & 0.03 & 0.04 & 0.05 & 0.03 & - & - \\
\hline $\mathrm{H}_{2} \mathrm{O}^{+}$ & - & - & 0.14 & - & 0.11 & - & 0.08 & - \\
\hline $\mathrm{H}_{2} \mathrm{O}^{-}$ & - & - & - & - & - & - & - & - \\
\hline Total & 100.24 & 99.86 & 100.40 & 99.53 & 99.81 & 99.70 & 99.97 & 100.39 \\
\hline $\mathrm{Si}$ & 1.863 & 1.828 & 1.842 & 1.919 & 1.886 & 1.899 & 1.901 & 1.834 \\
\hline $\mathrm{Al}$ & 0.137 & 0.172 & 0.168 & 0.081 & 0.114 & 0.101 & 0.074 & 0.166 \\
\hline $\mathrm{Ti}$ & 0.006 & 0.022 & 0.009 & 0.018 & 0.009 & 0.022 & 0.020 & 0.020 \\
\hline $\mathrm{Al}$ & 0.119 & 0.111 & 0.108 & 0.073 & 0.088 & 0.074 & - & 0.031 \\
\hline $\mathrm{Fe}^{+3}$ & 0.039 & 0.039 & 0.026 & 0.031 & 0.018 & 0.022 & 0.044 & 0.070 \\
\hline $\mathrm{Cr}$ & 0.021 & 0.026 & 0.035 & 0.061 & 0.031 & 0.039 & 0.031 & 0.035 \\
\hline $\mathrm{Fe}$ & 0.078 & 0.083 & 0.086 & 0.077 & 0.079 & 0.068 & 0.112 & 0.123 \\
\hline $\mathrm{Mn}$ & 0.004 & 0.002 & 0.004 & 0.002 & 0.002 & 0.002 & 0.002 & 0.002 \\
\hline $\mathrm{Ni}$ & - & - & - & - & - & - & - & - \\
\hline $\mathrm{Mg}$ & 0.878 & 0.904 & 0.952 & 0.934 & 0.882 & 0.902 & 0.987 & 0.905 \\
\hline $\mathrm{Ca}$ & 0.776 & 0.732 & 0.706 & 0.743 & 0.847 & 0.756 & 0.820 & 0.783 \\
\hline $\mathrm{Na}$ & 0.100 & 0.100 & 0.082 & - & 0.048 & 0.149 & 0.022 & 0.044 \\
\hline $\mathbf{K}$ & 0.004 & 0.004 & 0.004 & - & - & 0.004 & - & - \\
\hline $2-\mathrm{Si}$ & 0.137 & 0.172 & 0.168 & 0.081 & 0.114 & 0.101 & 0.099 & 0.166 \\
\hline $\mathrm{Al} / \mathrm{Cr}$ & 8.8 & 10.8 & 8 & 2.5 & 6.5 & 4.4 & 2.4 & 5.6 \\
\hline
\end{tabular}

No. 17-25 Ross, C. E., Foster, M. D. and Myers, A. T. (1954) 
Before answering this question, it should be examined whether the clinopyroxenes of basic and ultrabasic intrusive rocks show the same tendency with respect to the relation between $2-\mathrm{Si}$ and $\mathrm{Al} / \mathrm{Cr}$ above mentioned or not.

The $\mathrm{Cr}_{2} \mathrm{O}_{3}$ contents in clinopyroxene from basic and ultrabasic intrusives, such as gabbros, peridotites, dunites and pyroxenites were reported by BRowN $(1957,1962)$, Hess $(1960)$ and ONUKI $(1963,1965,1966)$. Their data of clinopyroxenes are assembled in Table 13, and 2-Si and $\mathrm{Al} / \mathrm{Cr}$ relation is plotted in Fig. 11.

The present petrogenetic interest in these inclusions is whether they are subvolcanic crystallization products from basaltic magma or unmelted fragments of peridotite layer. As is clearly shown in Fig. 11, the clinopyroxenes in the early stage of crystallization of many basic and ultrabasic intrusive rocks indicates the same tendency as those of olivine-rich inclusions in basaltic rocks. However, it should be noted that 2-Si in clinopyroxenes of basic and ultrabasic intrusive rocks never exceeds 0.125 .

It is suspicious, however, whether all clinopyroxenes of intrusive peridotite and/or gabbros are formed directly from the host magma or some of them are relict of the original body from which the magma of intrusive peridotite and/or gabbros are formed.

As to the clinopyroxene of intrusive peridotite and/or gabbro, I am inclined to take the former interpretation for the reason discussed below.

If oxide and silicate minerals are crystallized in parallel from the same magma, $\mathrm{Cr}_{2} \mathrm{O}_{3}$ in magma, for example, must be fixed on both oxide and silicate minerals at constant ratios. Therefore, the contents of $\mathrm{Cr}_{2} \mathrm{O}_{3}$ in co-existing minerals must have changed with changing of $\mathrm{Cr}_{2} \mathrm{O}_{3}$ contents in the host magma. In this respect, if the rate of crystallization of oxide and silicate minerals from the magma is almost constant, the $\mathrm{Cr}_{2} \mathrm{O}_{3}$ contents of clinopyroxene and orthopyroxene can be expected to show a continuous smooth line in $\mathrm{Al}_{2} \mathrm{O}_{3}-\mathrm{Cr}_{2} \mathrm{O}_{3}$ or $\mathrm{Al} / \mathrm{Cr}$ and 2-Si diagram.

In the early stage of clinopyroxene crystallization of Miyamori, Horoman, Skargaard, Bushveld and Stiilwater intrusions, the $\mathrm{Al} / \mathrm{Cr}$ ratios remain constant, while in the middle and late stage, the $\mathrm{Al} / \mathrm{Cr}$ ratios increase rapidly, as shown in Fig. 11. In other words, the $\mathrm{Al} / \mathrm{Cr}$ variation lines are parallel to the 2-Si axis in the early stage, and then curved upwards to become almost vertical to the 2-Si axis in the middle and late stage.

The abrupt change of $\mathrm{Cr}_{2} \mathrm{O}_{3}$ contents in these clinopyroxene suggests that the rate of crystallization of oxide and silicate is changed at this stage. The reason is essentially due to abrupt increment of oxygen partial pressure in that magma. In such condition, $\mathrm{Cr}_{2} \mathrm{O}_{3}$ is subtracted in the form of chromite and/or picotite from the magma. As the result, $\mathrm{Al} / \mathrm{Cr}$ in magma is rapidly increased with the formation of oxide minerals, whereas 2 -Si of pyroxene is almost unchanged during their formation.

The effect of oxygen partial pressure on rock melt is demonstrated experimentally by KATSURA (1967). His experiment was undertaken on natural rock samples under the condition of constant oxygen partial pressure and at various 
Table 13. Chemical composition of clinopyroxene from basic intrusive rocks

\begin{tabular}{|c|c|c|c|c|c|c|c|c|c|c|}
\hline No. & 1 & 2 & 3 & 4 & 5 & 6 & 7 & 8 & 9 & 10 \\
\hline $\mathrm{SiO}_{2}$ & 51.17 & 50.82 & 51.25 & 51.43 & 52.55 & 51.86 & 54.07 & 52.92 & 52.47 & 53.87 \\
\hline $\mathrm{TiO}_{2}$ & 0.97 & 0.96 & 0.76 & 0.27 & 0.24 & 0.55 & 0.21 & 0.50 & 0.46 & 0.07 \\
\hline $\mathrm{Al}_{2} \mathrm{O}_{3}$ & 3.22 & 2.40 & 2.92 & 3.00 & 2.98 & 2.33 & 2.08 & 2.80 & 2.28 & 1.64 \\
\hline $\mathrm{Cr}_{2} \mathrm{O}_{3}$ & 0.42 & 0.06 & 0.17 & 1.09 & 1.18 & 0.01 & 0.98 & 0.88 & 0.08 & 0.62 \\
\hline $\mathrm{Fe}_{2} \mathrm{O}_{3}$ & 1.53 & 1.88 & 1.70 & 1.37 & 1.17 & 1.60 & 0.56 & 0.85 & 1.49 & 0.67 \\
\hline $\mathrm{FeO}$ & 4.54 & 10.75 & 8.87 & 4.23 & 3.98 & 9.45 & 2.53 & 5.57 & 6.61 & 1.74 \\
\hline $\mathrm{MnO}$ & 0.13 & 0.19 & 0.18 & 0.14 & 0.12 & 0.24 & 0.09 & 0.15 & 0.20 & 0.05 \\
\hline $\mathrm{NiO}$ & - & 一 & - & - & 0.05 & - & 0.04 & 0.10 & 0.04 & - \\
\hline $\mathrm{MgO}$ & 16.68 & 14.36 & 15.04 & 19.44 & 18.21 & 14.50 & 17.39 & 16.40 & 15.71 & 17.39 \\
\hline $\mathrm{CaO}$ & 20.54 & 18.12 & 19.04 & 17.86 & 19.23 & 18.92 & 22.12 & 19.97 & 20.28 & 23.32 \\
\hline $\mathrm{Na}_{2} \mathrm{O}$ & 0.65 & 0.39 & 0.37 & 0.28 & 0.32 & 0.23 & 0.41 & 0.35 & 0.31 & 0.39 \\
\hline $\mathrm{K}_{2} \mathrm{O}$ & 0.05 & 0.03 & 0.05 & 0.02 & 0.00 & 0.00 & 0.00 & 0.01 & 0.02 & 0.02 \\
\hline $\mathrm{P}_{2} \mathrm{O}_{5}$ & - & - & - & - & - & - & - & - & - & - \\
\hline $\mathrm{H}_{2} \mathrm{O}^{+}$ & - & 一 & - & 0.70 & 0.14 & 0.37 & 0.04 & 0.10 & 0.20 & nd. \\
\hline $\mathrm{H}_{2} \mathrm{O}^{-}$ & - & - & - & 0.10 & 0.08 & 0.09 & 0.06 & 0.07 & 0.04 & nd. \\
\hline Total & 99.90 & 99.96 & 100.35 & 99.93 & 100.25 & 100.17 & 100.58 & 100.67 & 100.19 & 99.78 \\
\hline $\mathrm{Si}$ & 1.882 & 1.908 & 1.904 & 1.887 & 1.911 & 1.800 & 1.948 & 1.926 & 1.927 & 1.957 \\
\hline $\mathrm{Al}$ & 0.118 & 0.092 & 0.096 & 0.113 & 0.089 & 0.096 & 0.052 & 0.074 & 0.073 & 0.043 \\
\hline $\mathrm{Ti}$ & 0.027 & 0.025 & 0.022 & 0.009 & 0.007 & 0.017 & 0.006 & 0.013 & 0.013 & 0.002 \\
\hline $\mathrm{Al}$ & 0.021 & 0.014 & 0.031 & 0.015 & 0.033 & - & 0.039 & 0.044 & 0.042 & 0.027 \\
\hline $\mathrm{Fe}^{+3}$ & 0.042 & 0.054 & 0.047 & 0.040 & 0.035 & 0.042 & 0.017 & 0.026 & 0.040 & 0.018 \\
\hline $\mathrm{Cr}$ & 0.013 & 0.002 & 0.004 & 0.031 & 0.035 & - & 0.032 & 0.026 & 0.004 & 0.018 \\
\hline $\mathrm{Fe}$ & 0.139 & 0.336 & 0.277 & 0.128 & 0.122 & 0.275 & 0.076 & 0.170 & 0.203 & 0.053 \\
\hline $\mathrm{Mn}$ & 0.004 & 0.007 & 0.007 & 0.004 & 0.002 & 0.006 & 0.002 & 0.006 & 0.007 & 0.002 \\
\hline $\mathrm{Ni}$ & - & - & - & - & 0.002 & - & - & 0.002 & - & - \\
\hline $\mathrm{Mg}$ & 0.915 & 0.803 & 0.833 & 1.069 & 0.992 & 0.754 & 0.941 & 0.895 & 0.865 & 0.948 \\
\hline $\mathrm{Ca}$ & 0.809 & 0.729 & 0.759 & 0.704 & 0.748 & 0.704 & 0.852 & 0.779 & 0.797 & 0.908 \\
\hline $\mathrm{Na}$ & 0.046 & 0.029 & 0.027 & 0.022 & 0.022 & 0.012 & 0.026 & 0.026 & 0.022 & 0.027 \\
\hline $\mathrm{K}$ & 0.002 & 0.001 & 0.002 & - & - & - & - & - & - & - \\
\hline $2-\mathrm{Si}$ & 0.118 & 0.092 & 0.096 & 0.113 & 0.089 & 0.200 & 0.052 & 0.074 & 0.073 & 0.043 \\
\hline $\mathrm{Al} / \mathrm{Cr}$ & 10.6 & 53.0 & 29.5 & 4.1 & 3.5 & large & 2.0 & 4.5 & 26.0 & 3.8 \\
\hline
\end{tabular}

No. 1 Brown, G. M. (1957)

No. 2 Brown, G. M. (1960)

No. 3 Brown, G. M. (1957)

No. 4-9 HESS, H. H. (1960) 


\section{K. ISHIBASHI}

Table 13. continued

\begin{tabular}{|c|c|c|c|c|c|c|c|c|c|c|}
\hline No. & 11 & 12 & 13 & 14 & 15 & 16 & 17 & 18 & 19 & 20 \\
\hline $\mathrm{SiO}_{2}$ & 53.70 & 53.11 & 52.86 & 52.70 & 52.82 & 52.90 & 52.60 & 52.37 & 52.66 & 52.10 \\
\hline $\mathrm{TiO}_{2}$ & 0.16 & 0.25 & 0.38 & 0.36 & 0.27 & 0.35 & 0.37 & 0.46 & 0.48 & 0.52 \\
\hline $\mathrm{Al}_{2} \mathrm{O}_{3}$ & 1.62 & 2.20 & 2.65 & 2.62 & 2.58 & 2.41 & 2.61 & 3.01 & 2.90 & 3.06 \\
\hline $\mathrm{Cr}_{2} \mathrm{O}_{3}$ & 0.42 & 0.48 & 0.38 & 0.38 & 0.42 & 0.38 & 0.38 & 0.38 & 0.35 & 0.16 \\
\hline $\mathrm{Fe}_{2} \mathrm{O}_{3}$ & 0.63 & 0.85 & 0.90 & 0.94 & 0.97 & 0.99 & 0.95 & 0.75 & 0.74 & 1.16 \\
\hline $\mathrm{FeO}$ & 2.15 & 2.01 & 2.06 & 2.14 & 2.08 & 2.04 & 2.19 & 2.29 & 2.91 & 3.40 \\
\hline $\mathrm{MnO}$ & 0.05 & 0.09 & 0.10 & 0.09 & 0.09 & 0.12 & 0.10 & 0.13 & 0.13 & 0.14 \\
\hline $\mathrm{NiO}$ & - & - & - & - & - & - & - & - & - & - \\
\hline $\mathrm{MgO}$ & 17.10 & 17.01 & 16.95 & 16.98 & 16.97 & 16.98 & 16.95 & 16.49 & 16.09 & 15.13 \\
\hline $\mathrm{CaO}$ & 23.92 & 23.27 & 23.23 & 23.58 & 22.22 & 23.28 & 23.51 & 23.79 & 23.29 & 23.96 \\
\hline $\mathrm{Na}_{2} \mathrm{O}$ & 0.24 & 0.31 & 0.31 & 0.33 & 0.34 & 0.35 & 0.30 & 0.26 & 0.34 & 0.26 \\
\hline $\mathrm{K}_{2} \mathrm{O}$ & 0.04 & 0.03 & 0.02 & 0.06 & 0.03 & 0.04 & 0.06 & 0.04 & 0.07 & 0.03 \\
\hline $\mathrm{P}_{2} \mathrm{O}_{5}$ & - & - & - & - & - & 一 & - & - & - & - \\
\hline $\mathrm{H}_{2} \mathrm{O}^{+}$ & nd. & 0.11 & 0.01 & 0.00 & 0.05 & nd. & 0.02 & 0.07 & 0.05 & 0.03 \\
\hline $\mathrm{H}_{2} \mathrm{O}^{-}$ & nd. & 0.05 & 0.09 & 0.03 & 0.00 & nd. & 0.05 & 0.03 & 0.00 & 0.02 \\
\hline Total & 100.03 & 99.77 & 99.94 & 100.21 & 99.84 & 99.84 & 100.09 & 100.07 & 100.01 & 99.97 \\
\hline $\mathrm{Si}$ & 1.952 & 1.937 & 1.924 & 1.917 & 1.925 & 1.926 & 1.918 & 1.909 & 1.922 & 1.915 \\
\hline $\mathrm{Al}$ & 0.048 & 0.063 & 0.076 & 0.083 & 0.075 & 0.074 & 0.082 & 0.091 & 0.078 & 0.085 \\
\hline $\mathrm{Ti}$ & 0.004 & 0.007 & 0.010 & 0.010 & 0.007 & 0.010 & 0.010 & 0.012 & 0.013 & 0.014 \\
\hline $\mathrm{Al}$ & 0.021 & 0.032 & 0.038 & 0.029 & 0.036 & 0.029 & 0.030 & 0.038 & 0.046 & 0.047 \\
\hline $\mathrm{Fe}^{+3}$ & 0.017 & 0.023 & 0.024 & 0.026 & 0.027 & 0.027 & 0.026 & 0.021 & 0.020 & 0.032 \\
\hline $\mathrm{Cr}$ & 0.012 & 0.014 & 0.011 & 0.011 & 0.012 & 0.010 & 0.011 & 0.011 & 0.010 & 0.005 \\
\hline $\mathrm{Fe}$ & 0.065 & 0.061 & 0.062 & 0.062 & 0.063 & 0.062 & 0.066 & 0.070 & 0.088 & 0.104 \\
\hline $\mathrm{Mn}$ & 0.002 & 0.003 & 0.003 & 0.003 & 0.003 & 0.004 & 0.003 & 0.004 & 0.004 & 0.004 \\
\hline $\mathrm{Ni}$ & - & - & - & - & 一 & - & - & - & - & - \\
\hline $\mathrm{Mg}$ & 0.932 & 0.930 & 0.925 & 0.926 & 0.927 & 0.927 & 0.927 & 0.902 & 0.881 & 0.834 \\
\hline $\mathrm{Ca}$ & 0.931 & 0.909 & 0.906 & 0.919 & 0.906 & 0.912 & 0.918 & 0.930 & 0.911 & 0.944 \\
\hline $\mathrm{Na}$ & 0.017 & 0.022 & 0.022 & 0.023 & 0.024 & 0.024 & 0.021 & 0.018 & 0.024 & 0.019 \\
\hline $\mathbf{K}$ & 0.002 & 0.001 & 0.001 & 0.003 & 0.001 & 0.002 & 0.003 & 0.002 & 0.003 & 0.001 \\
\hline 2-Si & 0.048 & 0.063 & 0.076 & 0.083 & 0.075 & 0.074 & 0.082 & 0.091 & 0.078 & 0.085 \\
\hline $\mathrm{Al} / \mathrm{Cr}$ & 5.7 & 6.8 & 10.3 & 10.2 & 10.0 & 10.3 & 10 & 11 & 12.4 & 26 \\
\hline
\end{tabular}

No. 10-31 ONUKI, H. (1965)

No. 32-40 ONUKI, H. (1963) 
Table 13. continued

\begin{tabular}{|c|c|c|c|c|c|c|c|c|c|c|}
\hline No. & 21 & 22 & 23 & 24 & 25 & 26 & 27 & 28 & 29 & 30 \\
\hline $\mathrm{SiO}_{2}$ & 26 & 51.42 & 50.04 & 49.57 & 52.87 & 52.86 & 52.30 & 52.07 & 52.06 & 1.67 \\
\hline $\mathrm{TiO}_{2}$ & 0.70 & 0.69 & 0.72 & 0.86 & 0.12 & 11 & 0.15 & 0.15 & 0.25 & 0.23 \\
\hline $\mathrm{Al}_{2} \mathrm{O}_{3}$ & 3.80 & 3.07 & 4.52 & 4.31 & 2.54 & 2.91 & 3.95 & 3.58 & 4.00 & 4.04 \\
\hline $\mathrm{Cr}_{2} \mathrm{O}_{3}$ & 0.10 & 0.10 & 0.10 & 0.09 & 0.93 & 0.89 & 1.05 & 1.06 & .25 & .01 \\
\hline $\mathrm{Fe}_{2} \mathrm{O}_{3}$ & 2.16 & 1.99 & 1.91 & 2.30 & 0.65 & 0.69 & 0.75 & 0.73 & 0.86 & 0.94 \\
\hline $\mathrm{FeO}$ & 3.07 & 6.28 & 7.30 & 7.46 & 1.95 & 1.91 & 1.96 & 2.07 & 2.12 & 2.22 \\
\hline $\mathrm{MnO}$ & 0.15 & 0.18 & 0.21 & 0.27 & 0.06 & 0.07 & 0.08 & 0.08 & 0.12 & 0.09 \\
\hline $\mathrm{NiO}$ & - & - & -. & - & - & - & - & - & - & - \\
\hline $\mathrm{MgO}$ & 15.29 & 12.99 & 11.99 & 11.79 & 17.97 & 17.81 & 17.11 & 16.81 & 16.64 & 16.50 \\
\hline $\mathrm{CaO}$ & 22.99 & 22.72 & 22.66 & 22.81 & 22.30 & 22.18 & 21.87 & 22.36 & 21.45 & 22.15 \\
\hline $\mathrm{Na}_{2} \mathrm{O}$ & 0.49 & 0.53 & 0.49 & 0.48 & 0.38 & 0.39 & 0.45 & 0.47 & 0.64 & 0.58 \\
\hline $\mathrm{K}_{2} \mathrm{O}$ & 0.04 & 0.04 & 0.05 & 0.04 & 0.01 & 0.01 & 0.01 & 0.01 & 0.02 & 0.02 \\
\hline $\mathrm{P}_{2} \mathrm{O}_{5}$ & - & - & - & - & - & - & - & - & - & - \\
\hline $\mathrm{H}_{2} \mathrm{O}^{+}$ & nd. & 0.03 & nd. & 0.02 & 0.12 & nd. & nd. & 0.15 & nd. & 0.14 \\
\hline $\mathrm{H}_{2} \mathrm{O}^{-}$ & nd. & 0.10 & nd. & 0.00 & 0.07 & nd. & nd. & 0.11 & nd. & 0.11 \\
\hline Total & 100.05 & 100.14 & 99.99 & 100.00 & 99.97 & 99.83 & 99.68 & 99.65 & 99.41 & 99.70 \\
\hline $\mathrm{Si}$ & 1.880 & 1.912 & 1.871 & 1.861 & 1.921 & 1.918 & 1.902 & 1.904 & 1.900 & 1.890 \\
\hline $\mathrm{Al}$ & 0.120 & 0.088 & 0.129 & 0.139 & 0.079 & 0.082 & 0.098 & 0.096 & 0.100 & 0.110 \\
\hline $\mathrm{Ti}$ & & & 0.020 & 0.024 & 0.003 & 0.003 & 0.004 & 0.004 & 07 & 0.006 \\
\hline $\mathrm{Al}$ & 0.044 & 0.046 & 0.070 & 0.058 & 0.030 & 0.042 & 0.077 & 0.068 & 0.072 & 0.064 \\
\hline $\mathrm{Fe}^{+3}$ & 0.059 & 0.055 & 0.053 & 0.065 & 0.018 & 0.019 & 0.021 & 0.020 & 23 & 0.026 \\
\hline $\mathrm{Cr}$ & 0.003 & 0.003 & 0.003 & 0.003 & 0.027 & 0.026 & 0.030 & 0.030 & 0.036 & 0.029 \\
\hline $\mathrm{Fe}$ & 0.094 & 0.195 & 0.228 & 0.232 & 0.059 & 0.058 & 0.059 & 0.063 & 0.064 & 0.068 \\
\hline $\mathrm{Mn}$ & 0.005 & 0.006 & 0.007 & 0.009 & 0.002 & 0.002 & 0.002 & 0.002 & 0.004 & 0.003 \\
\hline $\mathrm{Ni}$ & - & - & - & - & - & - & - & - & - & - \\
\hline $\mathrm{Mg}$ & 0.841 & 0.724 & 0.673 & 0.654 & 0.978 & 0.969 & 0.933 & 0.922 & 911 & 0.905 \\
\hline $\mathrm{Ca}$ & 0.904 & 0.905 & 0.908 & 0.918 & 0.868 & 0.862 & 0.852 & 0.876 & 0.839 & 0.868 \\
\hline $\mathrm{Na}$ & 0.035 & 0.038 & 0.035 & 0.035 & 0.027 & 0.028 & 0.032 & 0.033 & 0.045 & 0.041 \\
\hline $\mathrm{K}$ & 0.002 & 0.002 & 0.002 & 0.002 & 0.000 & 0.000 & 0.000 & 0.000 & 0.001 & 0.001 \\
\hline $2-\mathrm{Si}$ & 0.120 & 0.088 & 0.129 & 0.139 & 0.079 & 0.082 & 0.098 & 0.096 & 0.100 & 0.110 \\
\hline $\mathrm{Al} / \mathrm{Cr}$ & 55 & 45 & 66 & 66 & 4.0 & 4.7 & 5.7 & 5.1 & 4.8 & 6 \\
\hline
\end{tabular}




\section{K. IsHIBASHI}

Table 13. continued

\begin{tabular}{|c|c|c|c|c|c|c|c|c|c|c|}
\hline No. & 31 & 32 & 33 & 34 & 35 & 36 & 37 & 38 & 39 & 40 \\
\hline $\mathrm{SiO}_{2}$ & 50.06 & 52.29 & 52.69 & 51.88 & 51.98 & 51.52 & 51.63 & 51.05 & 51.24 & 51.39 \\
\hline $\mathrm{TiO}_{2}$ & 0.78 & 0.13 & 0.14 & 0.31 & 0.23 & 0.35 & 0.33 & 0.28 & 0.32 & 0.34 \\
\hline $\mathrm{Al}_{2} \mathrm{O}_{3}$ & 2.89 & 2.74 & 2.09 & 4.17 & 3.37 & 3.67 & 3.64 & 5.15 & 4.01 & 4.19 \\
\hline $\mathrm{Cr}_{2} \mathrm{O}_{3}$ & 0.07 & 0.24 & 0.29 & 0.28 & 0.30 & 0.11 & 0.25 & 0.10 & 0.16 & 0.05 \\
\hline $\mathrm{Fe}_{2} \mathrm{O}_{3}$ & 3.52 & 1.78 & 1.25 & 0.97 & 0.92 & 1.18 & 1.69 & 1.89 & 1.83 & 1.80 \\
\hline $\mathrm{FeO}$ & 6.48 & 2.14 & 2.75 & 2.86 & 3.09 & 3.67 & 3.68 & 4.08 & 4.92 & 5.23 \\
\hline $\mathrm{MnO}$ & 0.54 & 0.12 & 0.12 & 0.13 & 0.08 & 0.09 & 1.10 & 0.10 & 0.11 & 0.12 \\
\hline $\mathrm{NiO}$ & - & - & - & - & - & - & - & - & - & - \\
\hline $\mathrm{MgO}$ & 13.63 & 18.62 & 17.33 & 16.71 & 16.54 & 16.53 & 16.91 & 16.41 & 16.43 & 15.38 \\
\hline $\mathrm{CaO}$ & 20.99 & 20.07 & 22.61 & 22.18 & 22.23 & 21.48 & 21.45 & 19.86 & 20.24 & 20.70 \\
\hline $\mathrm{Na}_{2} \mathrm{O}$ & 0.47 & 0.47 & 0.39 & 0.27 & 0.28 & 0.35 & 0.26 & 0.43 & 0.40 & 0.37 \\
\hline $\mathrm{K}_{2} \mathrm{O}$ & 0.01 & 0.07 & 0.02 & 0.03 & 0.05 & 0.03 & 0.01 & 0.06 & 0.03 & 0.10 \\
\hline $\mathrm{P}_{2} \mathrm{O}_{5}$ & - & - & - & - & - & - & - & - & - & - \\
\hline $\mathrm{H}_{2} \mathrm{O}^{+}$ & 0.15 & 0.32 & 0.18 & 0.13 & 0.38 & 0.32 & 0.11 & 0.11 & 0.11 & 0.20 \\
\hline $\mathrm{H}_{2} \mathrm{O}^{-}$ & 0.05 & 0.40 & 0.14 & 0.25 & 0.19 & 0.26 & 0.09 & 0.12 & 0.06 & 0.25 \\
\hline Total & 99.66 & 99.39 & 100.00 & 100.17 & 99.64 & 99.56 & 100.15 & 99.64 & 99.86 & 100.12 \\
\hline $\mathrm{Si}$ & 1.881 & 1.919 & 1.929 & 1.893 & 1.913 & 1.900 & 1.887 & 1.873 & 1.885 & 1.894 \\
\hline $\mathrm{Al}$ & 0.119 & 0.081 & 0.071 & 0.107 & 0.087 & 0.100 & 0.113 & 0.127 & 0.115 & 0.106 \\
\hline $\mathrm{Ti}$ & 0.022 & 0.004 & 0.004 & 0.008 & 0.006 & 0.010 & 0.009 & 0.008 & 0.009 & 0.010 \\
\hline $\mathrm{Al}$ & 0.009 & 0.037 & 0.019 & 0.072 & 0.059 & 0.061 & 0.061 & 0.085 & 0.059 & 0.076 \\
\hline $\mathrm{Fe}^{+3}$ & 0.099 & 0.049 & 0.035 & 0.027 & 0.025 & 0.033 & 0.047 & 0.052 & 0.051 & 0.050 \\
\hline $\mathrm{Cr}$ & 0.002 & 0.007 & 0.008 & 0.008 & 0.009 & 0.003 & 0.007 & 0.003 & 0.004 & 0.002 \\
\hline $\mathrm{Fe}$ & 0.203 & 0.066 & 0.084 & 0.087 & 0.095 & 0.113 & 0.112 & 0.125 & 0.151 & 0.161 \\
\hline $\mathrm{Mn}$ & 0.017 & 0.004 & 0.004 & 0.004 & 0.002 & 0.003 & 0.003 & 0.003 & 0.004 & 0.004 \\
\hline $\mathrm{Ni}$ & - & - & - & - & - & - & - & - & - & - \\
\hline $\mathrm{Mg}$ & 0.768 & 1.017 & 0.945 & 0.908 & 0.907 & 0.908 & 0.921 & 0.897 & 0.901 & 0.844 \\
\hline $\mathrm{Ca}$ & 0.845 & 0.789 & 0.886 & 0.867 & 0.876 & 0.848 & 0.840 & 0.780 & 0.798 & 0.817 \\
\hline $\mathrm{Na}$ & 0.034 & 0.033 & 0.028 & 0.019 & 0.020 & 0.025 & 0.018 & 0.031 & 0.028 & 0.026 \\
\hline $\mathrm{K}$ & 0.000 & 0.003 & 0.001 & 0.001 & 0.002 & 0.001 & 0.000 & 0.003 & 0.001 & 0.005 \\
\hline $2-\mathrm{Si}$ & 0.119 & 0.081 & 0.071 & 0.107 & 0.087 & 0.100 & 0.113 & 0.127 & 0.115 & 0.106 \\
\hline $\mathrm{Al} / \mathrm{Cr}$ & 64 & 17 & 11 & 23 & 16 & 57 & 24 & 71 & 44 & 91 \\
\hline
\end{tabular}




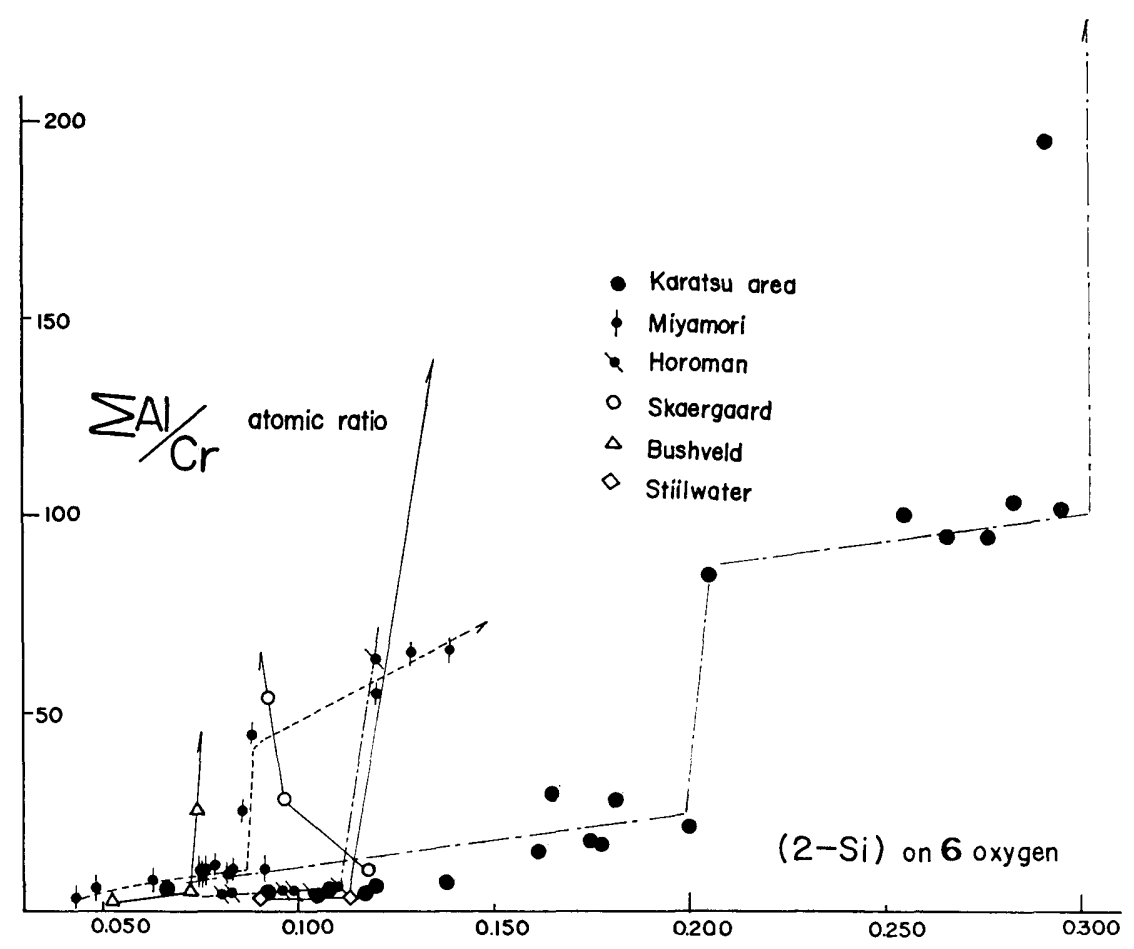

Fig. 11. The relation between $\mathrm{Al}-\mathrm{Cr}$ and 2-Si in clinopyroxenes from basic and ultrabasic inclusions. The clinopyroxenes in basic and ultrabasic inclusions from Karatsu area are shown for comparison.

temperatures by using a controlled furnace. When starting material is oceanite the following conclusion is drawn.

(a) When oxygen partial pressure is about $10^{-8}-10^{-4} \mathrm{~atm}$., and temperature is $1400^{\circ}-1350^{\circ} \mathrm{C}$, olivine is crystallized first from the melt, and upon lowering of the temperature, the crystallization may be followed successively by spinel, plagioclase, clinopyroxene and finally by orthopyroxene.

(b) When oxygen partial pressure is about $10^{-4}-10^{-1} \mathrm{~atm}$., and temperature is about $1400^{\circ}-1350^{\circ} \mathrm{C}$, spinel appears first, being followed by olivine and plagioclase, and finally, when the temperature is lowered down to $1150^{\circ} \mathrm{C}$, clinopyroxene and orthopyroxene are crystallized simultaneously from the melt.

(c) At higher oxygen partial pressure (up to $10^{-1} \mathrm{~atm}$.), hematite appears first and plagioclase and clinopyroxene follow with lowering temperature.

Judging from KATSURA's result (1967), it should be noted that under high oxygen partial pressure, $\mathrm{Fe}_{2} \mathrm{O}_{3}$ and $\mathrm{Cr}_{2} \mathrm{O}_{3}$ tend to be incooperated into oxide minerals rather than silicate minerals. As shown in Fig. 11, the point where the line is curved, indicates that the formation of oxide mineral is promoted rapidly by the increment of oxygen partial pressure in the magma.

d) Orthopyroxene

1) Chemical composition of orthopyroxene

The orthopyroxene is not so abundant as clinopyroxene in basic and ultrabasic inclusions. The separation of pure sample is the most tedious work. Six 
Table 14. Chemical composition of orthopyroxene (anal. K. Ishibashi)

\begin{tabular}{|c|c|c|c|c|c|c|c|c|c|c|}
\hline No. & $\begin{array}{c}1 \\
(8-D)\end{array}$ & $\stackrel{2}{2}$ & $\stackrel{3}{(17-D)}$ & $\begin{array}{c}4 \\
(15-D)\end{array}$ & $\stackrel{5}{5}$ & 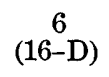 & $\stackrel{7}{7}$ & $\stackrel{8}{(7-D)}$ & $\begin{array}{c}9 \\
(4-D)\end{array}$ & $\begin{array}{c}10 \\
(5-D)\end{array}$ \\
\hline $\mathrm{SiO}_{2}$ & 48.13 & 49.28 & 49.36 & 49.81 & 50.62 & 50.92 & 50.93 & 51.73 & 53.43 & 54.56 \\
\hline $\mathrm{TiO}_{2}$ & 0.21 & 0.36 & 0.48 & 0.43 & 0.24 & 0.12 & 0.52 & 0.16 & 0.01 & 0.05 \\
\hline $\mathrm{Al}_{2} \mathrm{O}_{3}$ & 8.32 & 6.64 & 6.29 & 6.18 & 4.41 & 6.41 & 6.13 & 4.47 & 3.81 & 3.34 \\
\hline $\mathrm{Cr}_{2} \mathrm{O}_{3}$ & 0.31 & 0.14 & 0.13 & 0.03 & 0.31 & 0.04 & 0.15 & 0.28 & 0.50 & 0.56 \\
\hline $\mathrm{Fe}_{2} \mathrm{O}_{3}$ & 2.81 & 6.03 & 4.21 & 3.12 & 2.67 & 2.14 & 1.76 & 1.08 & 2.32 & 1.93 \\
\hline $\mathrm{FeO}$ & 7.11 & 13.04 & 10.78 & 10.65 & 8.38 & 8.33 & 9.72 & 8.25 & 4.43 & 4.18 \\
\hline $\mathrm{NiO}$ & 0.05 & nd. & 0.17 & 0.04 & 0.13 & 0.14 & 0.09 & 0.14 & 0.10 & 0.10 \\
\hline $\mathrm{MnO}$ & 0.13 & 0.23 & 0.28 & 0.18 & 0.20 & 0.24 & 0.21 & 0.21 & 0.15 & 0.15 \\
\hline $\mathrm{MgO}$ & 24.26 & 23.25 & 26.18 & 27.40 & 28.08 & 29.29 & 24.55 & 31.96 & 34.43 & 34.80 \\
\hline $\mathrm{CaO}$ & 8.50 & 0.45 & 1.58 & 1.49 & 4.06 & 2.12 & 5.28 & 1.63 & 0.61 & 0.41 \\
\hline $\mathrm{Na}_{2} \mathrm{O}$ & 0.45 & 0.39 & 0.25 & 0.09 & 0.25 & 0.21 & 0.38 & 0.14 & 0.07 & 0.07 \\
\hline $\mathrm{K}_{2} \mathrm{O}$ & 0.03 & 0.02 & 0.03 & 0.02 & 0.02 & 0.00 & 0.03 & 0.00 & 0.01 & 0.00 \\
\hline $\mathrm{P}_{2} \mathrm{O}_{5}$ & 0.00 & nd. & 0.01 & 0.01 & 0.00 & 0.00 & 0.00 & 0.00 & 0.01 & 0.00 \\
\hline $\mathrm{H}_{2} \mathrm{O}^{+}$ & 0.04 & nd. & 0.11 & 0.36 & 0.12 & 0.11 & 0.11 & 0.03 & 0.02 & 0.07 \\
\hline $\mathrm{H}_{2} \mathrm{O}^{-}$ & 0.03 & nd. & 0.08 & 0.24 & 0.04 & 0.08 & 0.03 & 0.01 & 0.02 & 0.04 \\
\hline Total & 100.38 & 99.83 & 99.94 & 100.05 & 99.53 & 100.15 & 99.89 & 100.09 & 99.92 & 100.26 \\
\hline $\mathrm{Si}$ & 1.725 & 1.795 & 1.783 & 1.794 & 1.818 & 1.806 & 1.891 & 1.822 & 1.855 & 1.884 \\
\hline $\mathrm{Al}$ & 0.275 & 0.205 & 0.217 & 0.206 & 0.182 & 0.194 & 0.168 & 0.178 & 0.145 & 0.116 \\
\hline $\mathrm{Cr}$ & - & - & - & - & - & - & - & - & - & - \\
\hline $\mathrm{Al}$ & 0.073 & 0.097 & 0.052 & 0.058 & 0.003 & 0.074 & 0.091 & 0.008 & 0.009 & 0.017 \\
\hline $\mathrm{Cr}$ & 0.009 & 0.004 & 0.002 & - & 0.013 & - & 0.004 & 0.008 & 0.012 & 0.017 \\
\hline $\mathrm{Fe}^{+3}$ & 0.077 & 0.171 & 0.113 & 0.082 & 0.078 & 0.055 & 0.047 & 0.030 & 0.058 & 0.050 \\
\hline $\mathrm{Ti}$ & 0.006 & 0.011 & 0.013 & 0.011 & 0.006 & 0.002 & 0.013 & 0.006 & - & 0.002 \\
\hline $\mathrm{Fe}$ & 0.213 & 0.396 & 0.325 & 0.322 & 0.252 & 0.245 & 0.291 & 0.243 & 0.127 & 0.120 \\
\hline $\mathrm{Ni}$ & 0.002 & - & 0.009 & - & 0.002 & 0.002 & 0.002 & 0.002 & 0.002 & 0.002 \\
\hline $\mathrm{Mn}$ & 0.002 & 0.007 & 0.004 & 0.006 & 0.006 & 0.006 & 0.006 & 0.006 & 0.004 & 0.004 \\
\hline $\mathrm{Mg}$ & 0.666 & 0.336 & 0.474 & 0.547 & 0.686 & 0.651 & 0.556 & 0.758 & 0.824 & 0.822 \\
\hline $\mathrm{Mg}$ & 0.639 & 0.934 & 0.942 & 0.933 & 0.826 & 0.906 & 0.796 & 0.931 & 0.969 & 0.981 \\
\hline $\mathrm{Ca}$ & 0.327 & 0.020 & 0.041 & 0.058 & 0.157 & 0.081 & 0.205 & 0.061 & 0.023 & 0.014 \\
\hline $\mathrm{Na}$ & 0.034 & 0.026 & 0.017 & 0.009 & 0.017 & 0.013 & 0.026 & 0.008 & 0.008 & 0.008 \\
\hline $\mathrm{K}$ & - & - & - & - & - & - & - & - & - & - \\
\hline $2-\mathrm{Si}$ & 0.275 & 0.205 & 0.217 & 0.206 & 0.182 & 0.194 & 0.168 & 0.178 & 0.145 & 0.116 \\
\hline $\mathrm{Al} / \mathrm{Cr}$ & 38.6 & 75.5 & 134.5 & large & 14.2 & - & 64.7 & 23.2 & 12.8 & 7.8 \\
\hline $\mathrm{Ca}$ & 16.9 & 1.2 & 2.3 & 3.0 & 7.4 & 4.2 & 11.0 & 3.1 & 0.8 & 1.5 \\
\hline $\mathrm{Mg}$ & 67.8 & 68.1 & 74.4 & 76.0 & 75.7 & 80.1 & 70.7 & 83.2 & 89.7 & 90.0 \\
\hline $\mathrm{Fe}$ & 15.3 & 30.7 & 23.4 & 21.0 & 16.9 & 15.7 & 18.3 & 13.7 & 9.5 & 8.5 \\
\hline
\end{tabular}




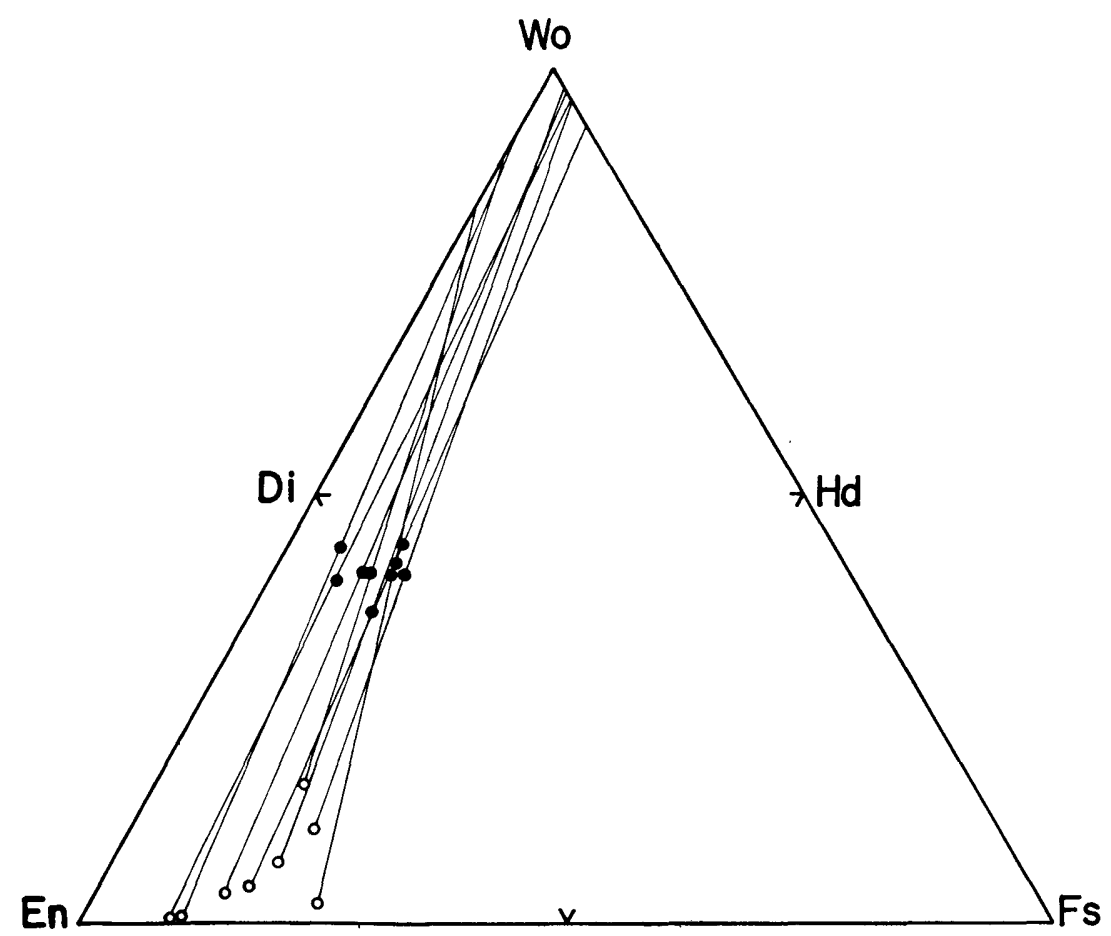

Fig. 12. Tie line relation between co-existing clinopyroxene and orthopyroxene.

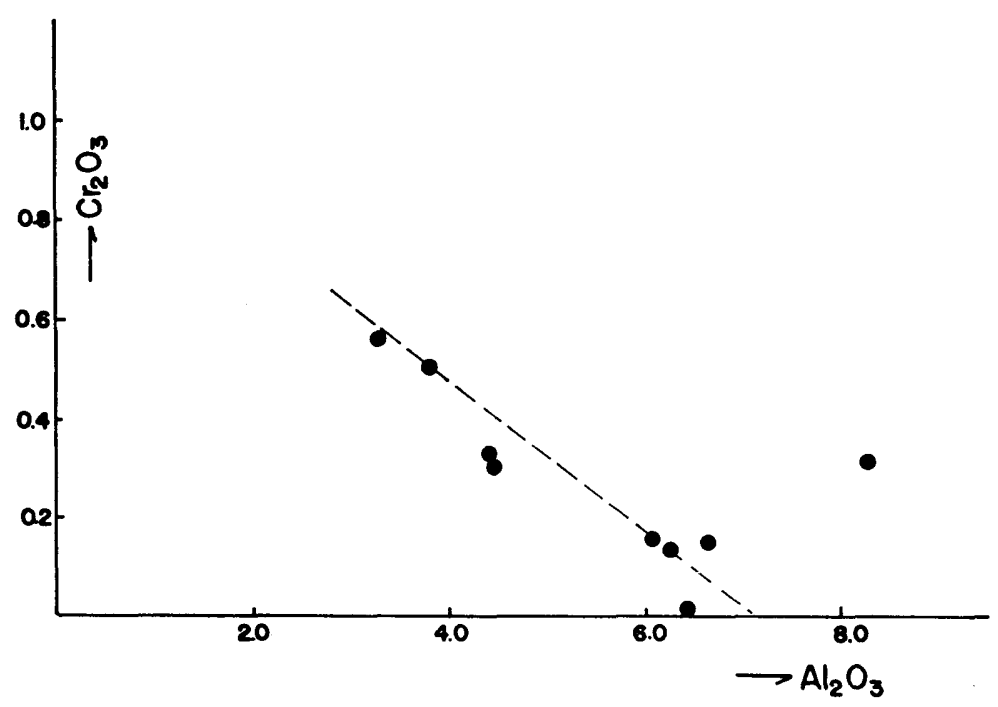

Fig. 13. The relation between $\mathrm{Cr}_{2} \mathrm{O}_{3}$ and $\mathrm{Al}_{2} \mathrm{O}_{3}$ in orthopyroxenes.

pure samples (Nos. 2-4, Nos. 8-10) of orthopyroxene and additional four samples (No. 1, Nos. 5-7) of orthopyroxene, the latter of which are mixed only a little amount of clinopyroxene, are chemically analyzed. The results are shown in Table 14. All of them co-exist with clinopyroxene. 


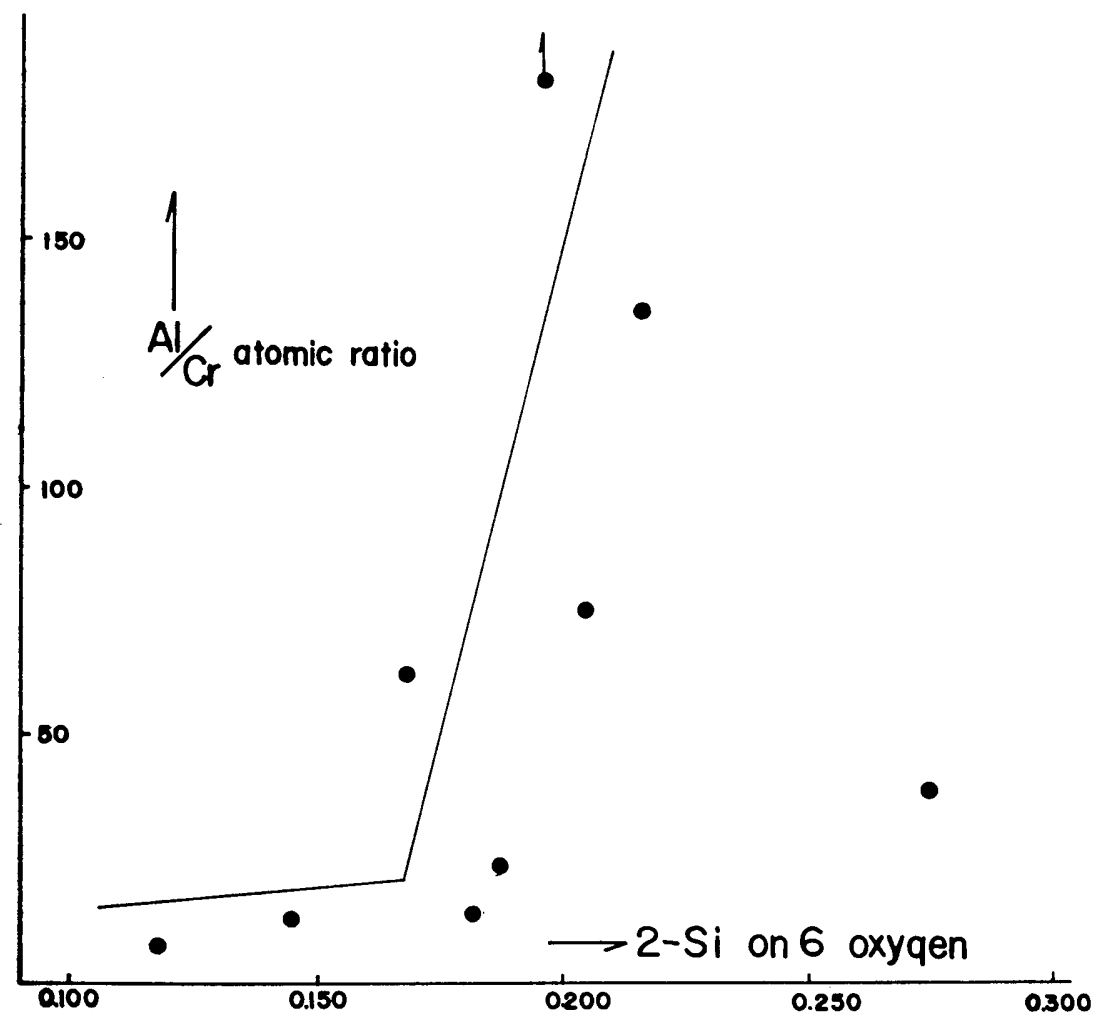

Fig. 14. $\mathrm{Al} / \mathrm{Cr}$ and $2-\mathrm{Si}$ relation in orthopyroxenes.

The tie line relation of co-existing pyroxenes (HESS, 1941) is shown in Fig. 12.

The relation between $\mathrm{Cr}_{2} \mathrm{O}_{3}$ and $\mathrm{Al}_{2} \mathrm{O}_{3}$ in analyzed orthopyroxene is also given in Fig. 13. The relation between $\mathrm{Al} / \mathrm{Cr}$ and 2-Si is shown in Fig. 14 .

As is clearly illustrated in Figs. 13 and 14, the relation between $\mathrm{Al}_{2} \mathrm{O}_{3}$ and $\mathrm{Cr}_{2} \mathrm{O}_{3}$ shows the same tendency as in the case of co-existing clinopyroxene. Also $2-\mathrm{Si}$ and $\mathrm{Al} / \mathrm{Cr}$ relation shows the same tendency as that in the co-existing pyroxenes (Fig. 10).

The $\mathrm{CaO}$ content in orthopyroxene was discussed by Kuno (1952) and HESS $(1941,1960)$. They have suggested that the $\mathrm{CaO}$ content of orthopyroxenes is the most useful parameter of temperature of crystallization. If chemical composition of host magma is maintained almost constant, $\mathrm{CaO}$ is more soluble in orthopyroxene solid solution at higher temperatures. Consequently the immiscible area of the co-existing pyroxenes becomes smaller at higher temperature. However, general tendency of this relation cannot be detected in this study.

Chemical composition of orthopyroxene (Table 14) and clinopyroxene (Table 10) are plotted on Wo-En-Fs diagram (Fig. 15). There is no remarkable difference between the orthopyroxenes of olivine-rich inclusions from Karatsu area and that from other localities.

2) The tie line relation of co-existing pyroxenes 


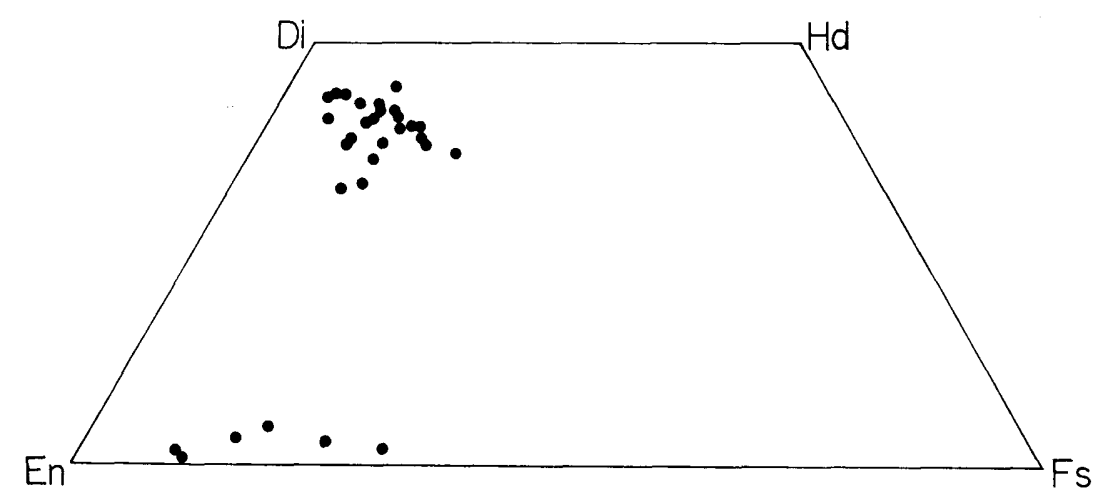

Fig. 15. Variation in composition of clinopyroxene and orthopyroxene in basic and ultrabasic inclusions in basaltic rocks. The orthopyroxenes of No. 1 , No. 5, No. 6 and No. 7 are excluded by contamination of clinopyroxene.

The tie line relation of co-existing pyroxenes was first discussed by Hess (1941). He has pointed out that the tie line of co-existing pyroxenes from igneous rock intersects at $\mathrm{Wo}_{75} \mathrm{En}_{25}$ on Wo-En composition line. HEss's tie line relation must have been resulted by iron and magnesium partition of co-existing pyroxenes. Figure 12 shows the tie line relation of co-existing pyroxenes of basic and ultrabasic inclusions and also phenocryst of basalt. As is illustrated in Fig. 12, the tie line of each pair of pyroxenes intersects Wo-En composition line at point between $\mathrm{Wo}_{80} \mathrm{En}_{20}$ and $\mathrm{Wo}_{100}$. It also intersects at point between $\mathrm{Wo}_{100}$ and $\mathrm{Wo}_{90} \mathrm{Fs}_{10}$ on Wo-Fs composition line. The location of the points where either Wo-En or Wo-Fs composition line is intersected by the tie line depends largely on the crystallization temperature of these crystals.

The theoretical consideration of this relation was made by MULLER (1960) and KeRTZ (1961). They considered that, if co-existing pyroxenes exist in perfect equilibrium in the rock, the iron and magnesium exchange reaction equilibrium is expressed in the following chemical equation:

$$
\mathrm{MgSiO}_{3}+\mathrm{CaFeSi}_{2} \mathrm{O}_{6} \leftrightharpoons \mathrm{CaMgSi}_{2} \mathrm{O}_{6}+\mathrm{FeSiO}_{3}
$$

If all pyroxene molecules are ideal solution, the partition coefficient (Kd) is defined in the following equation:

$$
\ln =\frac{x(1-y)}{(1-x) y}=\frac{\Delta G}{R T} \quad \frac{x}{(1-x)} \times \frac{(1-y)}{y}=\mathrm{e}^{-\frac{\Delta G}{R T}}
$$

$x=\mathrm{Fe} / \mathrm{Fe}+\mathrm{Mg}$ in orthopyroxene

$y=\mathrm{Fe} / \mathrm{Fe}+\mathrm{Mg}$ in clinopyroxene

In general, the $\mathrm{Kd}$ value of co-existing pyroxenes from metamorphic rocks is about 1.96-1.66, that of volcanic rocks is about $1.45-1.30$ and that of olivinerich inclusions in basaltic rocks is about 1.30-0.90 (MIYASHIRO, 1965). As pointed earlier, the $\mathrm{Kd}$ value of co-existing pyroxenes depends largely on temperature where the pyroxenes are crystallized, but the effect of pressure on $\mathrm{Kd}$ value is very small. The crystallization temperature becomes higher as Kd 
value of the co-existing pyroxenes becomes smaller. The $\mathrm{Kd}$ value of co-existing pyroxenes in basic and ultrabasic inclusions and in basaltic host rocks is calculated from chemical analyses, the result is shown in Table 15.

Table 15. The $\mathrm{Kd}$ value of co-existing pyroxenes in various kinds of inclusions in basaltic rocks

\begin{tabular}{|c|c|c|c|c|c|c|c|c|}
\hline \multirow{2}{*}{ No. } & \multicolumn{2}{|c|}{$\begin{array}{c}\text { ortho- } \\
\text { pyroxene }\end{array}$} & \multicolumn{2}{|c|}{$\begin{array}{c}\text { clino- } \\
\text { pyroxene }\end{array}$} & \multirow{2}{*}{\begin{tabular}{|l|} 
ortho-px. \\
$\mathrm{Fe} / \mathrm{Mg}+\mathrm{Fe}$ \\
\end{tabular}} & \multirow{2}{*}{$\frac{\text { clino-px. }}{\mathrm{Fe} / \mathrm{Mg}+\mathrm{Fe}}$} & \multirow{2}{*}{$\mathrm{Kd}$} & \\
\hline & $\mathrm{Mg}$ & $\mathrm{Fe}$ & $\mathrm{Mg}$ & $\mathrm{Fe}$ & & & & \\
\hline 1 & 34.43 & 4.43 & 18.07 & 2.33 & 0.114 & 0.114 & 1.00 & \multirow{3}{*}{$\begin{array}{l}\text { Cr-diopside } \\
\text { peridotite }\end{array}$} \\
\hline 2 & 34.80 & 4.18 & 18.01 & 2.45 & 0.107 & 0.119 & $0.89\}$ & \\
\hline 3 & 31.96 & 8.25 & 17.21 & 3.51 & 0.205 & 0.169 & 1.27 & \\
\hline 4 & 24.26 & 7.11 & 17.08 & 3.69 & 0.226 & 0.177 & $1.35)$ & \multirow{3}{*}{ Cr-pyroxenite } \\
\hline 5 & 28.08 & 8.38 & 17.54 & 5.87 & 0.229 & 0.250 & $0.85\}$ & \\
\hline 6 & 24.55 & 9.72 & 15.19 & 5.21 & 0.282 & 0.255 & 1.14 & \\
\hline 7 & 27.40 & 10.65 & 14.56 & 6.35 & 0.280 & 0.304 & 0.89 & Al-pyroxenite \\
\hline 8 & 29.29 & 8.33 & 15.11 & 4.79 & 0.221 & 0.240 & 0.90 & $\begin{array}{l}\text { Anorthite-bearing } \\
\text { Cr-pyroxenite }\end{array}$ \\
\hline 9 & 26.18 & 10.78 & 15.70 & 5.17 & 0.291 & 0.247 & 1.25 & Al-pyroxene gabbro \\
\hline 10 & 23.25 & 13.04 & 14.83 & 3.59 & 0.359 & 0.194 & 2.59 & Banded gabbro \\
\hline $11^{*}$ & 27.91 & 10.32 & 14.31 & 5.68 & 0.269 & 0.284 & 0.97 & Phenocryst \\
\hline
\end{tabular}

* Kuno, H. (1964)

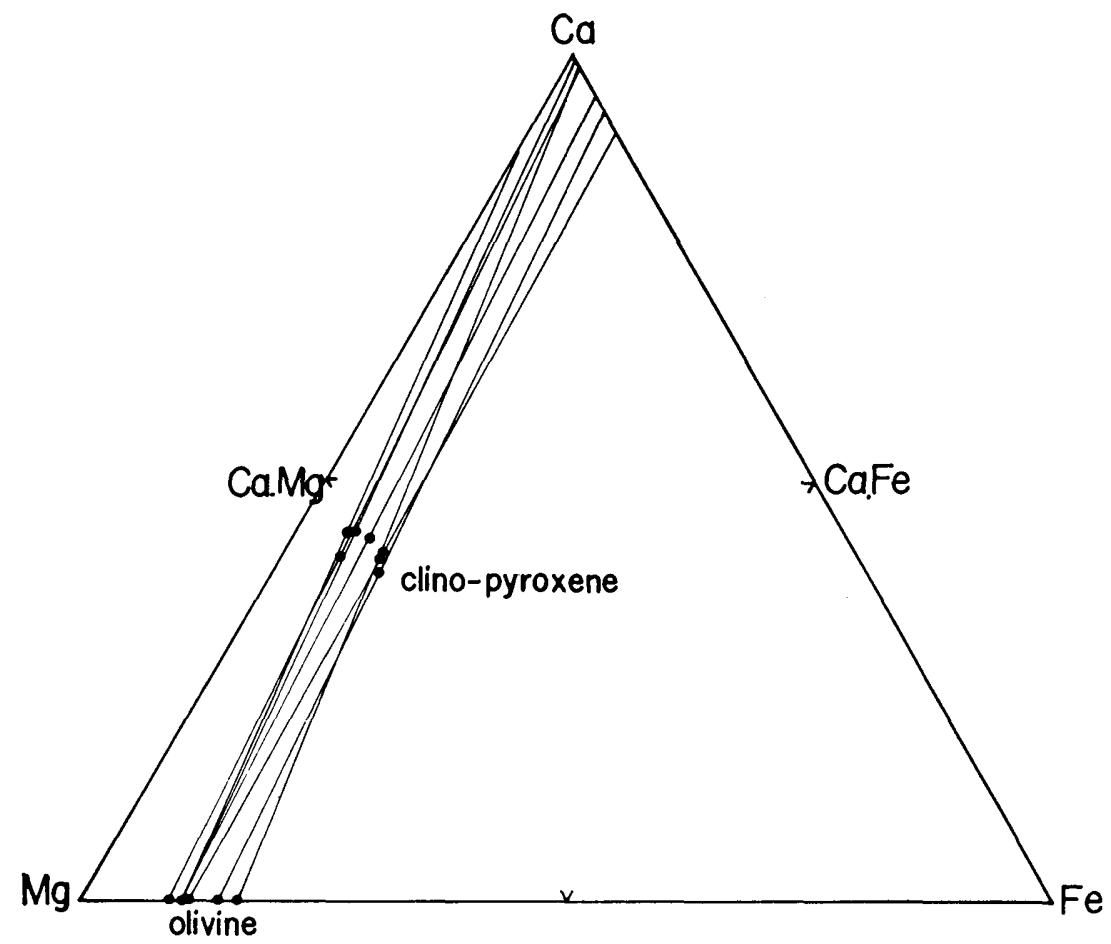

Fig. 16. Tie line relation between co-existing clinopyroxene and olivine. 


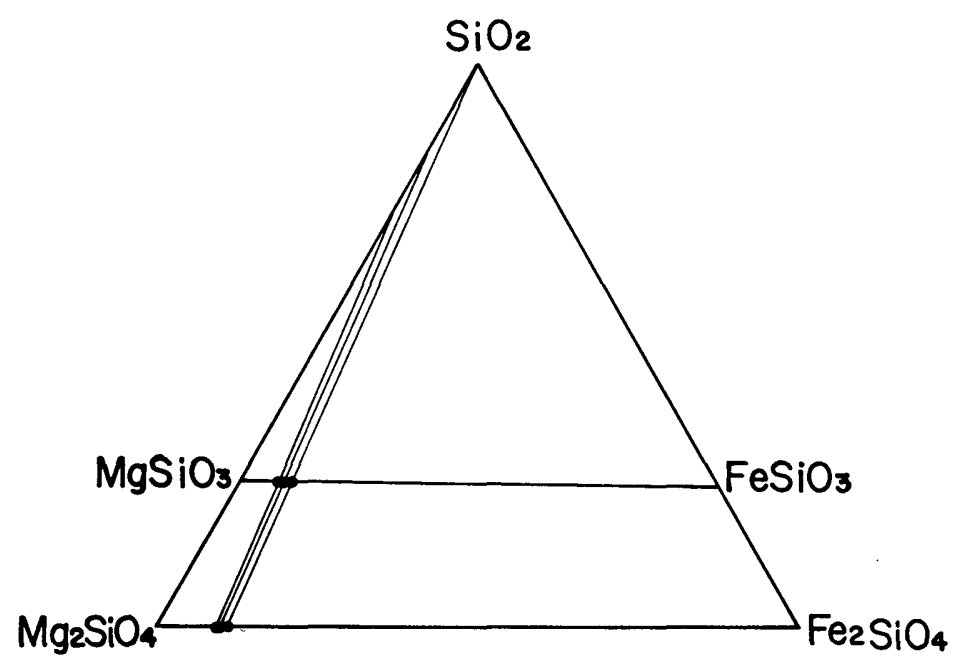

Fig. 17. Tie line relation of co-existing olivine and orthopyroxene.

In green type inclusions the $\mathrm{Kd}$ values range from 0.80 to 1.35 , and in black type inclusions it ranges from 0.89 to 2.50 . It is considered that temperature of co-existing pyroxenes crystallized in the green type inclusions is higher than that in black type inclusions.

In general, it is considered that sub-surface temperature of earth is increased with increasing depth. For this reason variation of Kd value seems to suggest that olivine-rich inclusions in basaltic rocks may have been brought up by the basaltic magma from different depth of earth crust and mantle.

3) The tie line relation of olivine and clinopyroxene and of olivine and orthopyroxene

The tie line relation of co-existing olivine and clinopyroxene and that of orthopyroxene and olivine are shown in Figs. 16 and 17 respectively. As illustrated in Figs. 16 and 17, the direction of tie line of each set of co-existing minerals shows the same tendency as that of co-existing pyroxenes.

e) Plagioclase

1) Chemical composition of plagioclase

Plagioclase is an important constituent minerals in gabbros and norites. In some Cr-pyroxenite, there is a small amount of bytownite or anothite. The data of chemical analyses of plagioclase are shown in Table 16 together with atomic ratios of elements based on $\mathrm{O}=8.000$. The Or-Ab-An ratios are plotted in the triangular diagram (Fig. 18). No.1 is in the anorthite-bearing Cr-pyroxenite from Takashima, No. 2 is in the banded gabbro from Kurose, No. 3 is in Al-pyroxene gabbro from Takashima, Nos. 4 and 5 are phenocrysts plagioclase in the basalt from Takashima. Nos. 1 and 2 are anorthite in composition, however, No. 3 is labradolite, the same in composition with the phenocrystic plagioclase in basaltic rocks.

Chemical analyses of plagioclase phenocrysts in basaltic rocks and their differentiated suite from northern Kyushu have also been made (IsHIBAsHI unpublished data). In Fig. 18, the solid circle indicates phenocrysts plagioclase 
Table 16. Chemical composition of plagioclase (anal. K. Ishibashi)

\begin{tabular}{|c|c|c|c|c|c|}
\hline No. & $\stackrel{1}{(16-\mathrm{E})}$ & $\stackrel{2}{2}-\mathrm{E})$ & $\begin{array}{c}3 \\
(17-E)\end{array}$ & $\begin{array}{c}4 \\
\left(19-E_{1}\right)\end{array}$ & $\begin{array}{c}5 \\
\left(19-\mathrm{E}_{2}\right)\end{array}$ \\
\hline $\mathrm{SiO}_{2}$ & 43.90 & 45.51 & 52.39 & 54.75 & 54.92 \\
\hline $\mathrm{TiO}_{2}$ & 0.00 & 0.00 & 0.00 & 0.00 & 0.00 \\
\hline $\mathrm{Al}_{2} \mathrm{O}_{3}$ & 36.18 & 35.40 & 30.39 & 28.03 & 28.85 \\
\hline $\mathrm{Cr}_{2} \mathrm{O}_{3}$ & nd. & nd. & nd. & nd. & nd. \\
\hline $\mathrm{Fe}_{2} \mathrm{O}_{3}$ & 0.42 & 0.01 & 0.42 & 0.80 & 0.45 \\
\hline $\mathrm{FeO}$ & 0.00 & nd. & 0.00 & 0.00 & 0.00 \\
\hline $\mathrm{NiO}$ & nd. & nd. & nd. & nd. & nd. \\
\hline $\mathrm{MnO}$ & 0.00 & 0.00 & 0.00 & 0.00 & 0.00 \\
\hline $\mathrm{MgO}$ & 0.03 & 0.01 & 0.02 & 0.02 & 0.04 \\
\hline $\mathrm{CaO}$ & 17.60 & 17.07 & 10.95 & 9.71 & 9.51 \\
\hline $\mathrm{Na}_{2} \mathrm{O}$ & 1.45 & 1.98 & 5.21 & 5.72 & 5.80 \\
\hline $\mathrm{K}_{2} \mathrm{O}$ & 0.16 & 0.01 & 0.51 & 0.52 & 0.61 \\
\hline $\mathrm{P}_{2} \mathrm{O}_{5}$ & 0.01 & 0.01 & 0.01 & 0.01 & 0.01 \\
\hline $\mathrm{H}_{2} \mathrm{O}^{+}$ & 0.14 & nd. & 0.21 & 0.38 & 0.11 \\
\hline $\mathrm{H}_{2} \mathrm{O}^{-}$ & 0.02 & nd. & 0.08 & 0.13 & 0.02 \\
\hline Total & 99.91 & 100.00 & 100.19 & 100.07 & 100.32 \\
\hline $\mathrm{Si}$ & 2.033 & 2.108 & 2.381 & 2.488 & 2.473 \\
\hline $\mathrm{Al}$ & 1.972 & 1.993 & 1.625 & 1.512 & 1.530 \\
\hline $\mathrm{Cr}$ & - & - & - & - & - \\
\hline $\mathrm{Fe}^{+3}$ & 0.017 & - & 0.016 & 0.014 & 0.016 \\
\hline $\mathrm{Mg}$ & - & - & - & - & - \\
\hline $\mathrm{Ca}$ & 0.872 & 0.876 & 0.534 & 0.471 & 0.459 \\
\hline $\mathrm{Na}$ & 0.133 & 0.092 & 0.458 & 0.502 & 0.508 \\
\hline $\mathrm{K}$ & 0.011 & - & 0.027 & 0.027 & 0.032 \\
\hline \multicolumn{6}{|c|}{$O=8.000$} \\
\hline An & 86 & 90 & 52.4 & 47 & 47.5 \\
\hline $\mathrm{Ab}$ & 13 & 10 & 45.0 & 50 & 49.0 \\
\hline Or & 1 & 0 & 2.6 & 3 & 3.5 \\
\hline
\end{tabular}

and open square shows normative composition of plagioclase calculated from bulk chemical composition of host rocks. They are tied up with a solid line. The broken line indicates the trend of feldspar composition of basaltic rocks and their differentiated suite from northern Kyushu. As is illustrated in Fig. 19, the plagioclase in Al-pyroxene gabbro is at the middle position of the analyzed plagioclase of basalt-hawaiite series from northern Kyushu. It must be noted that the chemical composition of plagioclase, orthopyroxene and clinopyroxene in Al-pyroxene gabbro shows a good concidence with phenocryst minerals in the basaltic rocks. This fact seems to suggest that the Al-pyroxene gabbro may have been formed by settling of crystals from basaltic magma in magma reservoir at an early stage. 


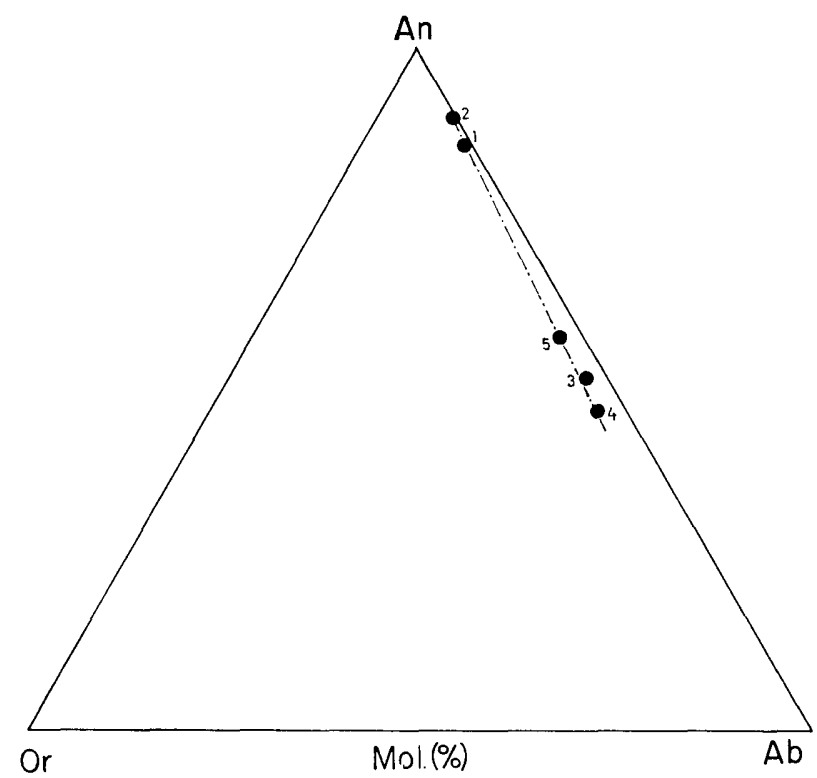

Fig. 18. Variation in composition of plagioclase from basic and ultrabasic inclusions in basaltic rocks. The sample numbers reffer to Table 16 .

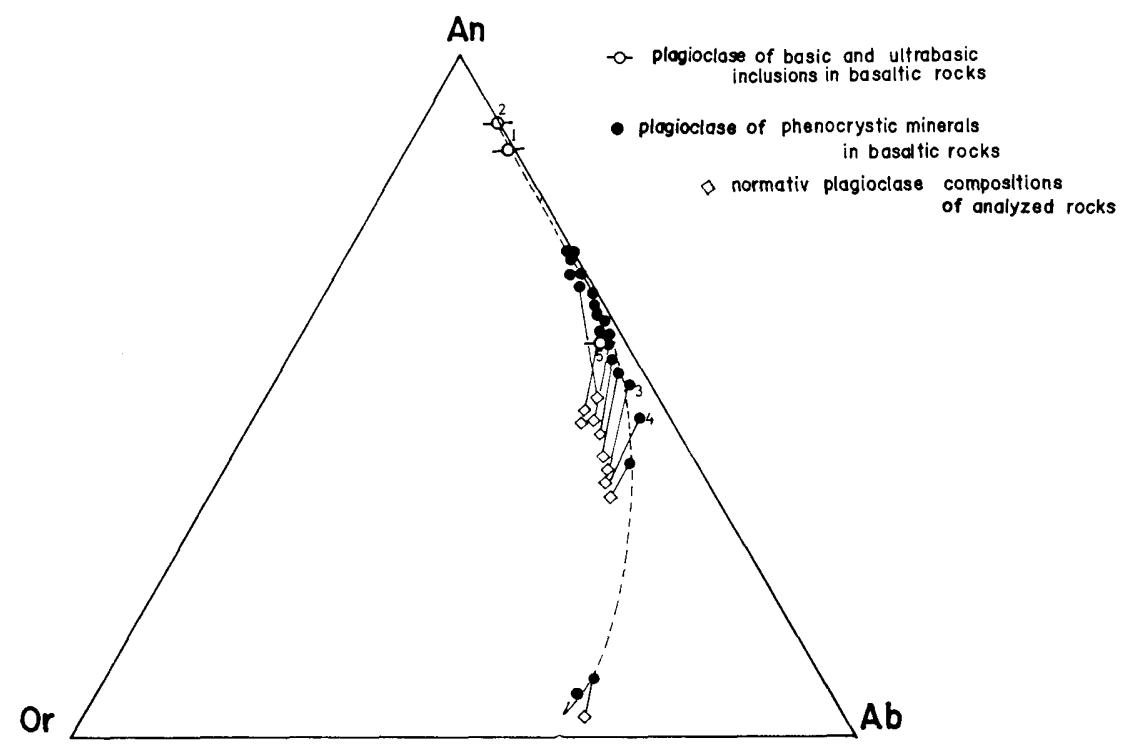

Fig. 19. The chemical composition of plagioclase in basic and ultrabasic inclusions and phenocrysts of basalts from northern Kyushu.

F. Bulk chemical composition of basic and ultrabasic inclusions in basaltic rocks

Five bulk analyses of basic and ultrabasic inclusions are shown in Table 17 (Nos. 1-5) with C. I. P. W. norm. Five bulk analyses of inclusion bearing basalts 
Table 17. Bulk chemical composition of basic and ultrabasic inclusions and their basaltic host rocks (anal. K. Ishibashi)

\begin{tabular}{|c|c|c|c|c|c|c|c|c|c|c|}
\hline No. & 1 & 2 & 3 & 4 & 5 & 6 & 7 & 8 & 9 & 10 \\
\hline $\mathrm{SiO}_{2}$ & 40.74 & 40.65 & 46.71 & 48.46 & 50.76 & 48.32 & 48.24 & 46.57 & 42.24 & 44.03 \\
\hline $\mathrm{TiO}_{2}$ & 0.17 & 0.37 & 0.22 & 0.30 & 0.55 & 1.69 & .54 & 89 & 1.72 & .84 \\
\hline $\mathrm{Al}_{2} \mathrm{O}_{3}$ & 1.48 & 1.73 & 15.27 & 2.19 & 51 & 16.04 & 14.03 & 11 & 13.57 & 11.26 \\
\hline $\mathrm{Fe}_{2} \mathrm{O}_{3}$ & 0.22 & 1.38 & 5.86 & 1.16 & 2.52 & 2.99 & 3.19 & 4.52 & .94 & 2.26 \\
\hline $\mathrm{FeO}$ & 9.25 & 10.98 & 3.93 & 7.45 & 4.04 & 6.48 & 7.38 & 36 & 31 & 11 \\
\hline $\mathrm{MnO}$ & 0.13 & 0.15 & 0.13 & 0.19 & 0.18 & 0.17 & 0.18 & 8 & 20 & 20 \\
\hline $\mathrm{MgO}$ & 47.94 & 44.45 & 12.26 & 26.63 & 18.30 & 9.97 & 11.53 & 19 & .10 & 16.37 \\
\hline $\mathrm{CaO}$ & 0.07 & 0.05 & 14.25 & 12.36 & 16.62 & 8.20 & 10.17 & 7 & 86 & .06 \\
\hline $\mathrm{Na}_{2} \mathrm{O}$ & 0.0 & 0.07 & 1.03 & 0.40 & & 2.98 & 2.50 & 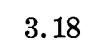 & 07 & 3.07 \\
\hline $\mathrm{K}_{2} \mathrm{O}$ & & 2 & 0 & & & & & & & 51 \\
\hline $\mathrm{P}_{2} \mathrm{O}_{5}$ & 0.01 & 0.01 & 0.01 & 0.01 & 0.01 & 0.44 & 0.24 & 4 & 23 & 0.94 \\
\hline $\mathrm{H}_{2} \mathrm{O}^{+}$ & 0.16 & 0.30 & 0.11 & 0.38 & 0.55 & 0.49 & 0.11 & .76 & .52 & 0.73 \\
\hline $\mathrm{H}_{2} \mathrm{O}^{-}$ & 0.03 & 0.07 & 0.15 & 0.13 & 0.27 & 0.33 & & & 95 & 0.67 \\
\hline Total & 100.29 & 100.23 & 100.02 & 99.68 & 100.00 & 99.78 & 100.36 & 99.69 & 99.57 & 100.05 \\
\hline or & 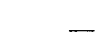 & - & & - & - & & & & & 90 \\
\hline$a b$ & 1.05 & 1.11 & 8.38 & 3.14 & 5.76 & 25.15 & 20.96 & 27.25 & 2.60 & 7.34 \\
\hline ne & - & - & - & - & - & - & - & - & 12.79 & 10.22 \\
\hline an & 0.28 & 0.28 & 36.97 & 3.34 & 11.97 & 25.30 & 23.91 & 13.07 & 17.78 & 12.51 \\
\hline $\mathrm{C}$ & 1.22 & 1.43 & - & - & - & - & - & - & - & - \\
\hline wo & - & - & 14.04 & 24.24 & 29.35 & 5.22 & 10.32 & 13.34 & 10.09 & 11.37 \\
\hline en & 0.50 & 6.70 & 24.50 & 27.80 & 36.60 & 4.90 & 8.40 & 12.10 & 7.30 & 8.20 \\
\hline fs & - & 0.92 & 1.72 & 5.15 & 3.04 & 1.72 & 1.98 & 3.17 & 1.85 & 2.11 \\
\hline fo & 84.18 & 73.60 & 4.34 & 27.16 & 44 & 14.00 & 14.28 & 5.88 & 18.34 & 22.82 \\
\hline $\mathrm{fa}$ & 12.85 & 13.67 & 0.20 & 5.71 & 1.22 & 4.08 & 5.30 & 1.63 & 5.10 & 6.53 \\
\hline $\mathrm{mt}$ & 0.23 & 1.86 & 8.58 & 1.86 & 3.71 & 4.41 & 4.64 & & 4.18 & 3.25 \\
\hline il & 0.46 & 1.16 & 0.46 & 0.61 & 1.22 & 3.19 & 2.89 & 3.65 & 3.04 & 3.50 \\
\hline ap & - & - & - & - & - & 1.01 & 0.67 & 1.34 & 2.69 & 2.02 \\
\hline
\end{tabular}

1 dunite inclusion from Takashima

2. dunite inclusion from Hinodematsu

3 olivine free gabbro from Kurose

4 peridotite inclusion from Hinodematsu

5 pyroxenite inclusion from Takashima

6 basalt: non-porphyritic part, from Takashima

7 basalt: slightly porphyritic with olivine and augite, from Takashima

8 basalt: slightly porphyritic with olivine, from Kurose

9 limburgite: slightly porphyritic with olivine and augite, from Ogusoyama

10 limburgite: non-porphyritic part, from Ogusoyama 


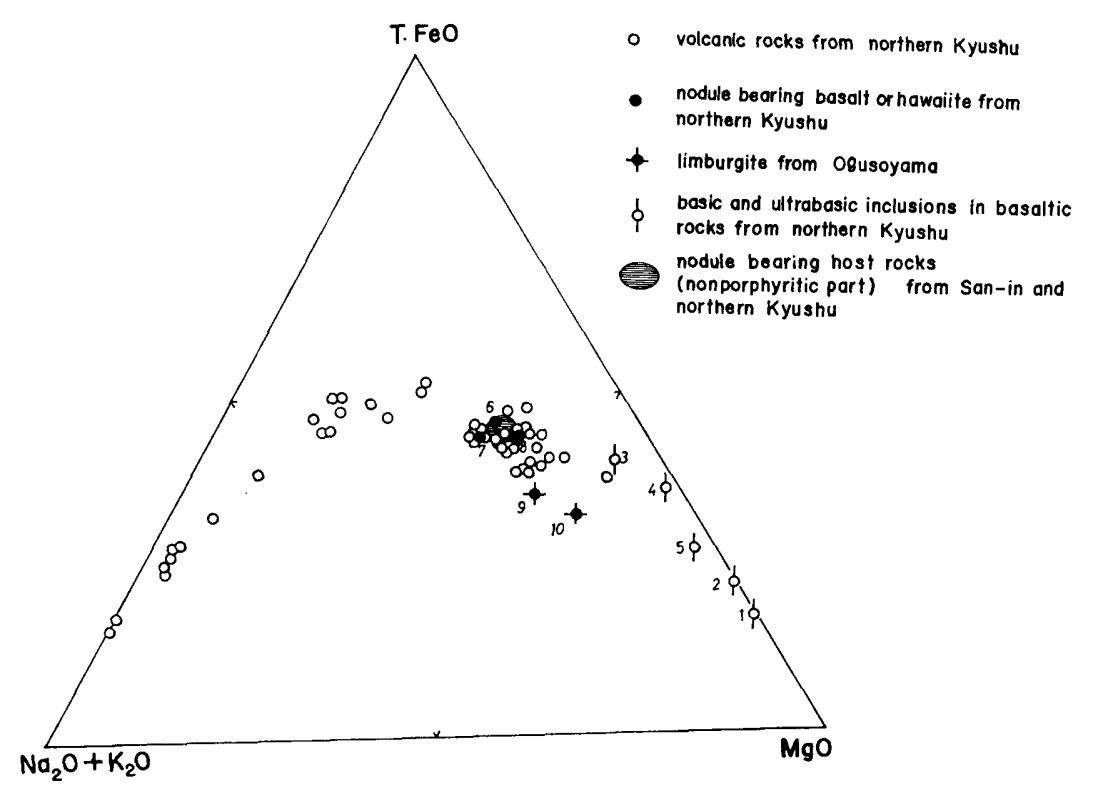

Fig. 20. $\mathrm{MgO}-\mathrm{T} \cdot \mathrm{FeO}-\left(\mathrm{Na}_{2} \mathrm{O}+\mathrm{K}_{2} \mathrm{O}\right)$ diagram showing the compositional distribution of basaltic rocks and their differentiated suite and of basic and ultrabasic inclusions from northern Kyushu.

and limburgite are also given in Table 17 (Nos. 6-10) with C. I. P. W. norm. They are plotted in $\mathrm{MgO}-\mathrm{T} . \mathrm{FeO}-\left(\mathrm{Na}_{2} \mathrm{O}+\mathrm{K}_{2} \mathrm{O}\right)$ diagram (Fig. 20). The data of chemical analyses of Cenozoic basaltic rocks and their differentiated suite are also plotted in the same diagram for comparison.

\section{Discussions}

A. Two distinct groups of inclusions in the Cenozoic basaltic rocks from the region of northern Kyushu and San-in

As has already been mentioned, the basic and ultrabasic inclusions in basaltic rocks can be divided into two groups by the color of pyroxenes. The differences of the two groups of inclusions are summarized in Table 18. The black type inclusion differs from the green type in the mode of occurrence, minerals paragenesis and chemical composition of constituent minerals.

Beside these two groups of inclusions, there are abundant large phenocrystic augite and hypersthene in the same basaltic rocks. MiYake (1948) reported that the zoned augite consisting black core and green margin, exists in alkali olivine basalt from Nishigatake, $30 \mathrm{~km}$ southwest of Takashima island. He suggested that the core was crystallized under certain physico-chemical condition, and the margin was formed under some different condition than that of the former.

There are numerous zoned crystals of clinopyroxenes in basalts from northern $\mathrm{Kyushu}$, the zoned crystals generally have green core rich in $\mathrm{Cr}_{2} \mathrm{O}_{3}$ and brownish black outer zone rich in $\mathrm{Al}_{2} \mathrm{O}_{3}$. If these zoned crystals are preserved at high temperature (below the melting point of the minerals) for a long time, the zoning 
Toble 18. Distinction of two types of inclusions

\begin{tabular}{|c|c|c|}
\hline Field name & green type inclusion & black type inclusion \\
\hline Rock type & $\begin{array}{l}\text { dunite, Cr-diopside peridotite, } \\
\text { Cr-pyroxenite, and anorthite- } \\
\text { bearing Cr-pyroxenite. }\end{array}$ & $\begin{array}{l}\text { olivine gabbro, olivine-free gab- } \\
\text { bro and Al-pyroxenite. }\end{array}$ \\
\hline Field occurrence & $\begin{array}{l}\text { some inclusion of this type are } \\
\text { surrounded by black type in- } \\
\text { clusion and mineral veins of } \\
\text { black type inclusion penetrates } \\
\text { through this type. }\end{array}$ & $\begin{array}{l}\text { clinopyroxene and orthopyro- } \\
\text { xene of the outermost part of } \\
\text { this inclusion show euhedral } \\
\text { outline. }\end{array}$ \\
\hline $\begin{array}{l}\text { Color of olivine } \\
\text { in nacked eye }\end{array}$ & yellowish green to light yellow. & yellowish green to green. \\
\hline $\begin{array}{l}\text { Color of pyroxene } \\
\text { in nacked eyes }\end{array}$ & light green to grass green. & pitch black to brownish black. \\
\hline $\begin{array}{l}\text { Miner inclusion } \\
\text { of oxide mineral } \\
\text { in silicate }\end{array}$ & chromite, rutile and magnetite. & $\begin{array}{l}\text { picotite-hercynite, ilmenite, } \\
\text { magnetite. }\end{array}$ \\
\hline $\begin{array}{l}\text { Optical } \\
\text { characteristics }\end{array}$ & $\begin{array}{l}\text { translation lamellae, andulate } \\
\text { extinction, minute striation and } \\
\text { exsolution lamellae of diopside } \\
\text { in enstatite crystal. }\end{array}$ & $\begin{array}{l}\text { translation lamellae, augite } \\
\text { shows purple color, some hy- } \\
\text { persthene decomposed to oli- } \\
\text { vine, pigeonite, plagioclase and } \\
\text { magnetite. }\end{array}$ \\
\hline $\begin{array}{l}\text { Chemical } \\
\text { characteristics }\end{array}$ & $\begin{array}{l}\text { constituent minerals of this } \\
\text { type characterized by } \mathrm{Cr}_{2} \mathrm{O}_{3} .\end{array}$ & $\begin{array}{l}\text { constituent minerals of this } \\
\text { type characterized by } \mathrm{Al}_{2} \mathrm{O}_{3} \text {. }\end{array}$ \\
\hline
\end{tabular}

would disappear and the minerals would become homogeneous crystals. As pointed by MiYAKe (1948), a sharp zoning in phenocryst pyroxenes indicates sudden change of physico-chemical condition under which the crystals were formed. The zoning also suggested that the crystals have not enough time to anneal the zoning before consolidation.

Decomposition of previously formed minerals is also induced by abrupt change of physico-chemical condition. In thin section, there are dense aggregate of maffic minerals in basalts which appear to be pseudomorphus after phenocrystic pyroxenes. A lot of crystals of large phenocrysts are decomposed at the margin. Some other crystals are decomposed completely to fine aggregates of olivine, augite, pigeonite, plagioclase and oxide minerals (Plate 23, Figs. 13 and 14). Existence of sponge augite and decomposed pyroxenes seem to suggest that most of the gigantic phenocrysts have xenocrystic aspect rather than ordinary phenocryst.

As pointed earlier in this paper, the chemistry of the large phenocrystic augite and hypersthene is closely related to augite and hypersthene of Alpyroxenite and gabbros. In addition, the following facts should be taken into consideration.

(1) All the basic and ultrabasic inclusions have a sharp boundary with basaltic and limburgitic host rocks.

(2) The clinopyroxene and orthopyroxene in some black type inclusions are eudhedral and have crystal faces of $a(100), m(110), b(010), u(111)$ and c(001) (Plate 18, Fig. 2). This fact indicates that the crystals of the black 
type inclusions are crystallized from basaltic magma.

(3) The crystals of the green type inclusions show equigranular texture sometimes having translation lamellae, and oriented arrangements of fine crystals of oxide minerals are often recognized in silicate minerals (Plate 22, Fig. 12).

(4) The green type inclusions are tranversed by minute veinlets of Al-augite and Al-hypersthene, while such veinlets could not be found in the black type inclusions.

(5) Spinel of the green type inclusions is always chromite. The oxide minerals of the black type inclusions are spinel or hercynite (Plate 25, Figs. 21, 22 and 24) and those of basaltic rocks are usually magnetite or titanomagnetite.

(6) Cr-diopside and Cr-enstatite occur in the green type inclusions, while in the black type inclusions, there are Al-augite and Al-hypersthene which resemble chemically and optically to the gigantic phenocrysts of augite inclusions as a reaction rim (Fig. 2; Plate 24, Figs. 17 and 18).

(7) Some of the green type inclusions are covered with minerals of black type inclusions as a reaction rim (Fig. 2; Plate 24, Figs. 17 and 18).

(8) The spongy augite and hypersthene (WILshire and Binns, 1961; YamaGUCHI, 1964) are always recognized in the basaltic host rocks as phenocrysts (Plate 23, Figs. 15 and 16; Plate 21, Fig. 7). They may have been derived from basic and ultrabasic inclusions.

(9) Constituent minerals of the both types of inclusions show undulated extinction, strongly so in green type inclusions. Some olivine and orthopyroxene have minute striation and irregular parting (Plate 22, Fig. 11). Olivine and orthopyroxene have translation lamellae or kink bands which must have been formed under a stressed condition in solid state.

(10) The $\mathrm{Fa}$ content of olivine in the green type inclusions is about $\mathrm{Fa}_{8-12}$, while that of olivine in the black type inclusions is about $\mathrm{Fa}_{15-25}$. The latter is almost the same in composition with the large phenocrystic olivine of basalt.

(11) $\mathrm{Al} / \mathrm{Cr}$ of clinopyroxenes is gradually increased with increasing 2-Si in the green type inclusions, however, there is a remarkable gap in composition between cliopyroxene of the green type inclusions and of the black type inclusions.

(12) WILKINSON (1956) pointed out that pigeonite and orthopyroxene were never crystallized from undersaturated basaltic magma. In the present study orthopyroxene is found both in the black type and the green type inclusions. KUNo (1964) has described the occurrence of Al-rich bronzite and hypersthene which are crystallized from alkali olivine basalt magma, and he emphasized that Al-rich bronzite and hypersthene can be crystallized directly from alkali olivine basalt magma at high pressure.

(13) The constituent minerals of the green type inclusions are characterized by higher content of $\mathrm{Cr}_{2} \mathrm{O}_{3}$ and lower content of $\mathrm{Al}_{2} \mathrm{O}_{3}$ and $\mathrm{TiO}_{2}$.

On these lines of evidences I lead to the conclusion that the green type inclusions are of deeper origin than the black type inclusions. 


\section{B. Relation between olivine-rich inclusions and basaltic host rock}

Basaltic magma is produced in the upper part of the mantle, although chemical and mineralogical composition of the mantle is not exactly known. If the basic and ultrabasic inclusions in basaltic rocks are assumed to have been caused by mechanical capturing of the deep seated rocks by basaltic magma on the way from subcrustal depth to the surface of the earth, we may be allowed to give the following discussions.

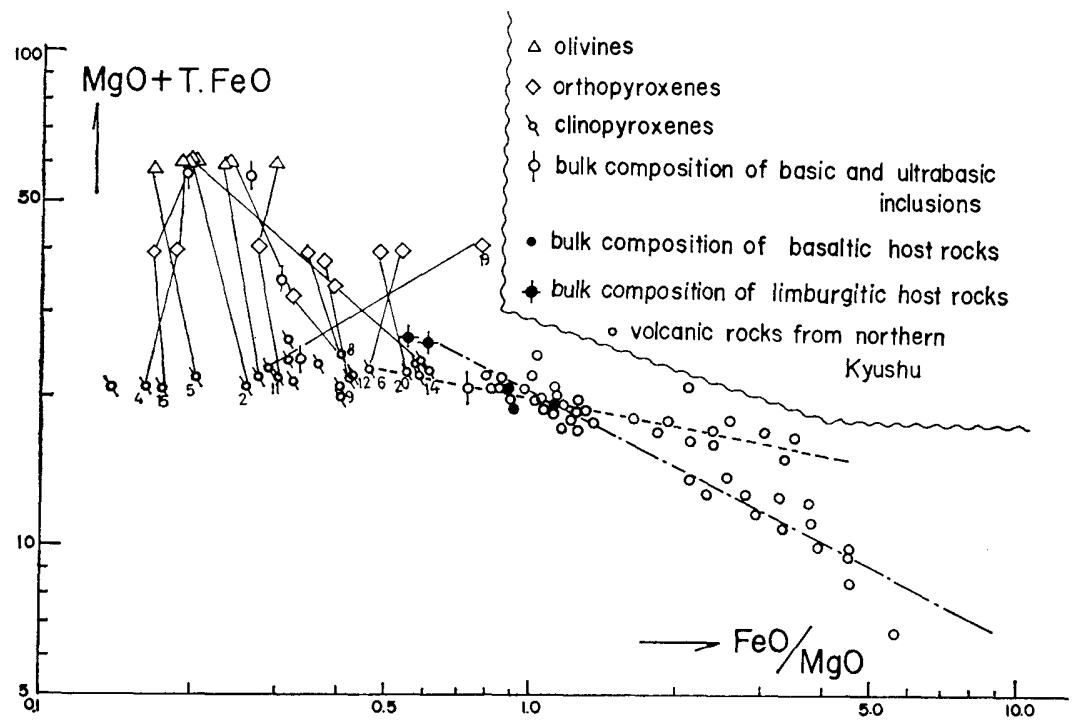

Fig. 21. The relation between $\mathrm{FeO}+\mathrm{MgO}$ and $\mathrm{T} \cdot \mathrm{FeO} / \mathrm{MgO}$ of the constituent minerals in basic and ultrabasic inclusions in basaltic rocks and their differentiated suite from northern Kyushu.

Figure 21 shows the relation between $\mathrm{MgO}+\mathrm{FeO}$ and $\mathrm{FeO} / \mathrm{MgO}$ of analyzed olivine, orthopyroxene and clinopyroxene. Figure 22 is essentially the same as Fig. 21, but is simplified to avoid confusion of lines and points. The inclusionbearing basaltic and limburgitic host rocks from northern Kyushu and from Ogusoyama are shown in the area of $(\mathrm{J})$ and $(\mathrm{K})$ in Fig. 22. In the same figure (P) represents the average chemical composition of peridotite inclusions given by Kushiro and Kuno (1963), which is shown in Table 19.

The line LL' shows the trend of crystallization of alkali olivine basalt magma of northern Kyushu, and is prolonged towards the left. It should be noted that line $L L^{\prime}$ passes through $(K)$ to $(P)$. On this fact I considered that $(J),(K)$ and $(\mathrm{P})$ have a compositional relation with respect to $\mathrm{FeO}$ and $\mathrm{MgO}$ which is explained below. KUSHIRO and KUNo (1963) pointed out that the partial melting of mantle peridotite is less than five per cent in volume. In Fig. 22, (P) represents the average chemical composition of peridotite inclusions and $(J)$ is the compositional area of inclusions-bearing basaltic host rocks which corresponds approximately to the liquid composition formed by partial melting of mantle peridotite. Now we can fix the point $(\mathrm{P})$ and $(\mathrm{K})$ on the line of LL' in Fig. 22, 


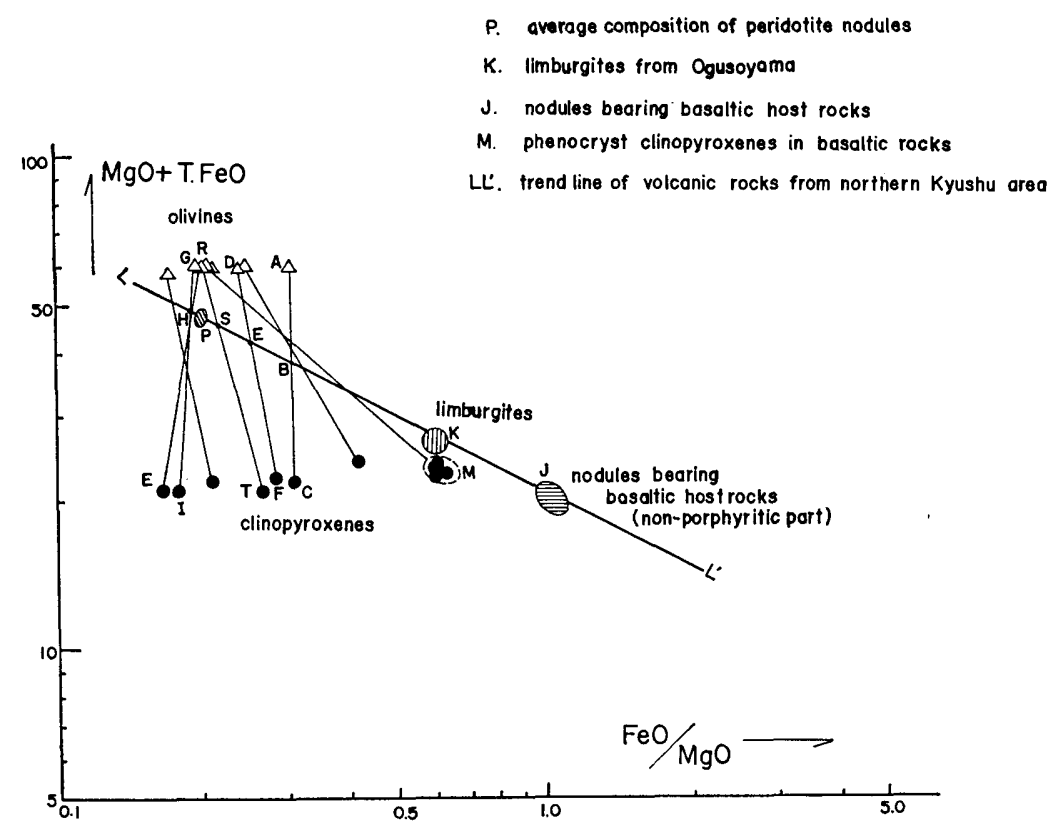

Fig. 22. Illusrative diagram for generation of basaltic magma by partial melting process from mantle peridotite.

Table 19. Average chemical composition of peridotite nodules in the world (KUshiro and Kuno, 1963)

\begin{tabular}{l|r}
$\mathrm{SiO}_{2}$ & 44.05 \\
$\mathrm{TiO}_{2}$ & 0.14 \\
$\mathrm{Al}_{2} \mathrm{O}_{3}$ & 2.80 \\
$\mathrm{Cr}_{2} \mathrm{O}_{3}$ & 0.36 \\
$\mathrm{Fe}_{2} \mathrm{O}_{3}$ & 1.16 \\
$\mathrm{FeO}$ & 7.13 \\
$\mathrm{MnO}$ & 0.14 \\
$\mathrm{MgO}$ & 40.98 \\
$\mathrm{CaO}$ & 2.49 \\
$\mathrm{Na}_{2} \mathrm{O}$ & 0.21 \\
$\mathrm{~K}_{2} \mathrm{O}$ & 0.03 \\
$\mathrm{P}_{2} \mathrm{O}_{5}$ & 0.02 \\
$\mathrm{H}_{2} \mathrm{O}^{+}$ & 0.36 \\
$\mathrm{H}_{2} \mathrm{O}^{-}$ & 0.08 \\
$\mathrm{Total}$ & \\
\hline
\end{tabular}

since (JP) indicates the total volume of liquid and crystal (the magma $J$ and peridotite $P$ ) in volume per cent. If a mantle peridotite forms liquid $(J)$ by about five per cent, the residual peridotite $(P)$ is about ninety-five per cent in volume, then the mantle peridotite can be determined at point $(E)$ on the line of $\mathrm{LL}^{\prime}$ which divides the line $L L^{\prime}$ into $\mathrm{EP}: \mathrm{EJ}=5: 95$. 
The mineralogical composition can be determined by each point of intersection at which the tie line crosses the line LL'. For example the composition of the point ( $\mathrm{E}$ ) is $\mathrm{FeO}: \mathrm{MgO}=9: 33$, in other words, it is the mixture of olivine and clinopyroxene whose composition is determined by the tie line (ED) and (EF), which is about 20:18 (olivine 18 and clinopyroxene 20). Table 20 shows the data of chemical composition of parental basaltic magmas in several areas. If we plot them in Fig. 22, they come in $(J)$ area. In spite of great variation in alkalies contents of above mentioned samples, the $\mathrm{FeO}$ and $\mathrm{MgO}$ contents are so constant that they are plotted within (J) area in Fig. 22.

Table 20. Chemical composition of parental basaltic magmas in several areas

\begin{tabular}{l|r|r|r|r|r}
\hline $\mathrm{No}$. & \multicolumn{1}{|c|}{1} & 2 & \multicolumn{1}{|c}{3} & \multicolumn{1}{|c}{4} & \multicolumn{1}{|c}{5} \\
\hline $\mathrm{SiO}_{2}$ & 48.73 & 47.76 & 47.12 & 46.48 & 48.38 \\
$\mathrm{TiO}_{2}$ & 0.63 & 0.91 & 1.83 & 2.70 & 1.36 \\
$\mathrm{Al}_{2} \mathrm{O}_{3}$ & 16.53 & 17.14 & 16.21 & 13.86 & 19.04 \\
$\mathrm{Fe}_{2} \mathrm{O}_{3}$ & 3.37 & 2.64 & 3.28 & 2.59 & 1.19 \\
$\mathrm{FeO}$ & 8.44 & 6.05 & 6.78 & 9.52 & 8.74 \\
$\mathrm{MnO}$ & 0.29 & 0.17 & 0.16 & 0.12 & 0.10 \\
$\mathrm{MgO}$ & 8.24 & 8.43 & 8.68 & 9.74 & 7.90 \\
$\mathrm{CaO}$ & 12.25 & 12.27 & 10.36 & 10.38 & 10.56 \\
$\mathrm{Na}_{2} \mathrm{O}$ & 1.21 & 2.33 & 2.27 & 2.82 & 2.46 \\
$\mathrm{~K}_{2} \mathrm{O}$ & 0.23 & 1.19 & 1.48 & 0.67 & 0.18 \\
$\mathrm{P}_{2} \mathrm{O}_{5}$ & 0.10 & 0.85 & 0.49 & 0.35 & 0.07 \\
$\mathrm{H}_{2} \mathrm{O}^{+}$ & - & 0.44 & 1.34 & 0.59 & - \\
$\mathrm{H}_{2} \mathrm{O}^{-}$ & - & 0.36 & - & 0.24 & - \\
$\mathrm{Total}$ & 100.02 & 100.54 & 100.00 & 100.06 & 99.98 \\
\hline
\end{tabular}
1) KUNO, H. (1951)
2) Томіта, T. (1951)
3) YAGI, K. (1958)
4) Kuno, H. et. al. (1957)
5) WAger, L. R. and DeER, W. A. (1939)

If the partial melting is taken place like this, the chemical composition of the residual peridotite, if we allow to call it so, must move towards $\mathrm{MgO}$ rich clinopyroxene as compared with the olivine and clinopyroxene of initial peridotite. Moreover, the volume of olivine increases more than that in the initial peridotite. When the partial melting proceeds further on, the residual peridotite becomes more richer in olivine content, changing finally to dunite.

The limburgite seems to be the mixture of parental basalt magma $(J)$ and peridotite (S) with volume proportion of about 6:4 (Fig. 22).

Abundant phenocrysts of olivine and augite in limburgite suggest that the bulk chemical composition of the rock does not imply the composition of liquid derived from the mantle peridotite by partial melting.

During the partial melting of mantle peridotite, the concentration of alkalies into liquid phase (parental basalt magma) is one of the most important problems for petrogenesis. The relation between total alkalies and silica in the consti- 


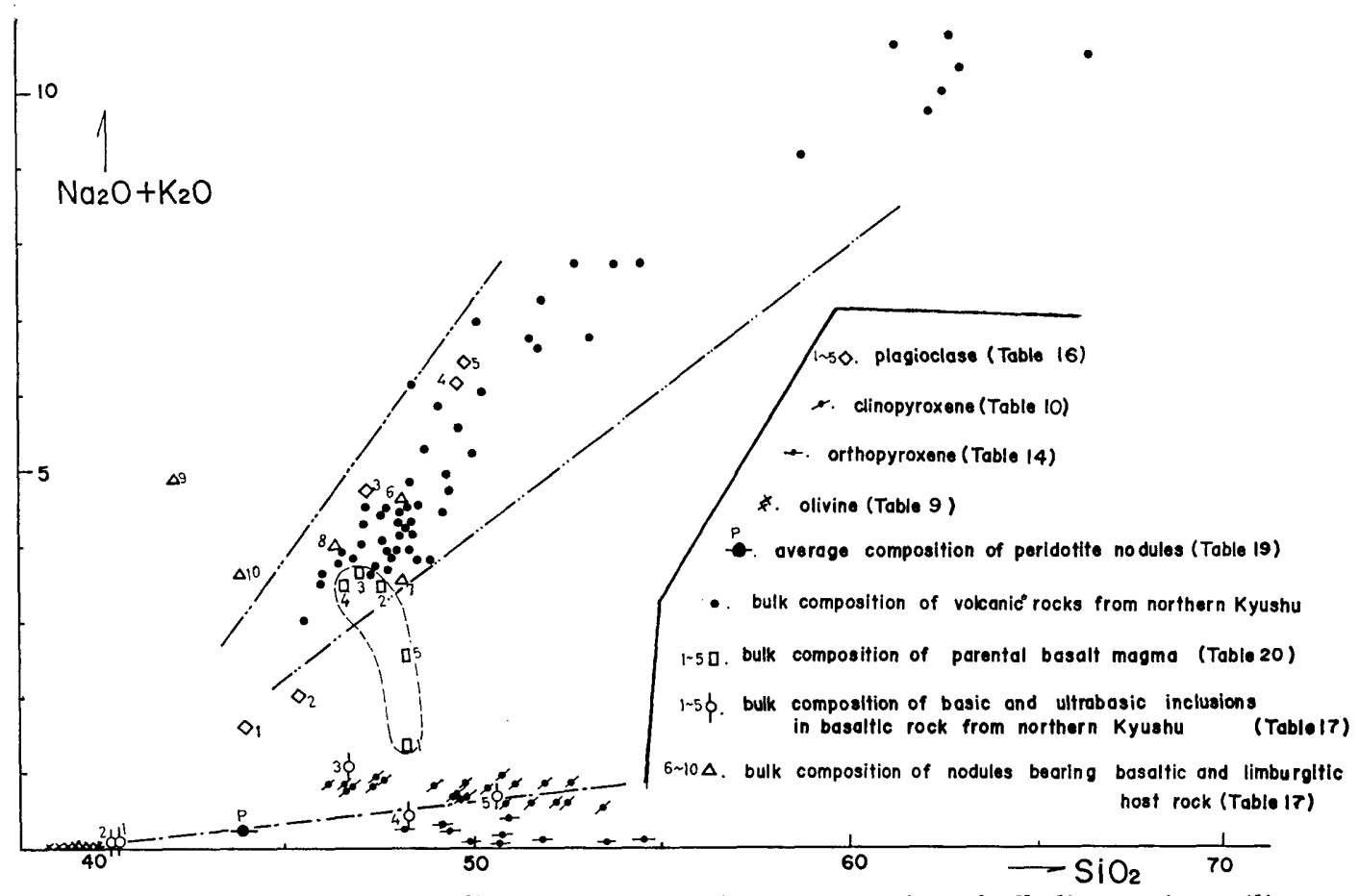

Fig. 23. $\mathrm{SiO}_{2}-\left(\mathrm{Na}_{2} \mathrm{O}+\mathrm{K}_{2} \mathrm{O}\right)$ diagram showing the concentration of alkalies against silica in various type of inclusions and their constituent minerals and also in basaltic rocks and their differentiated suite.

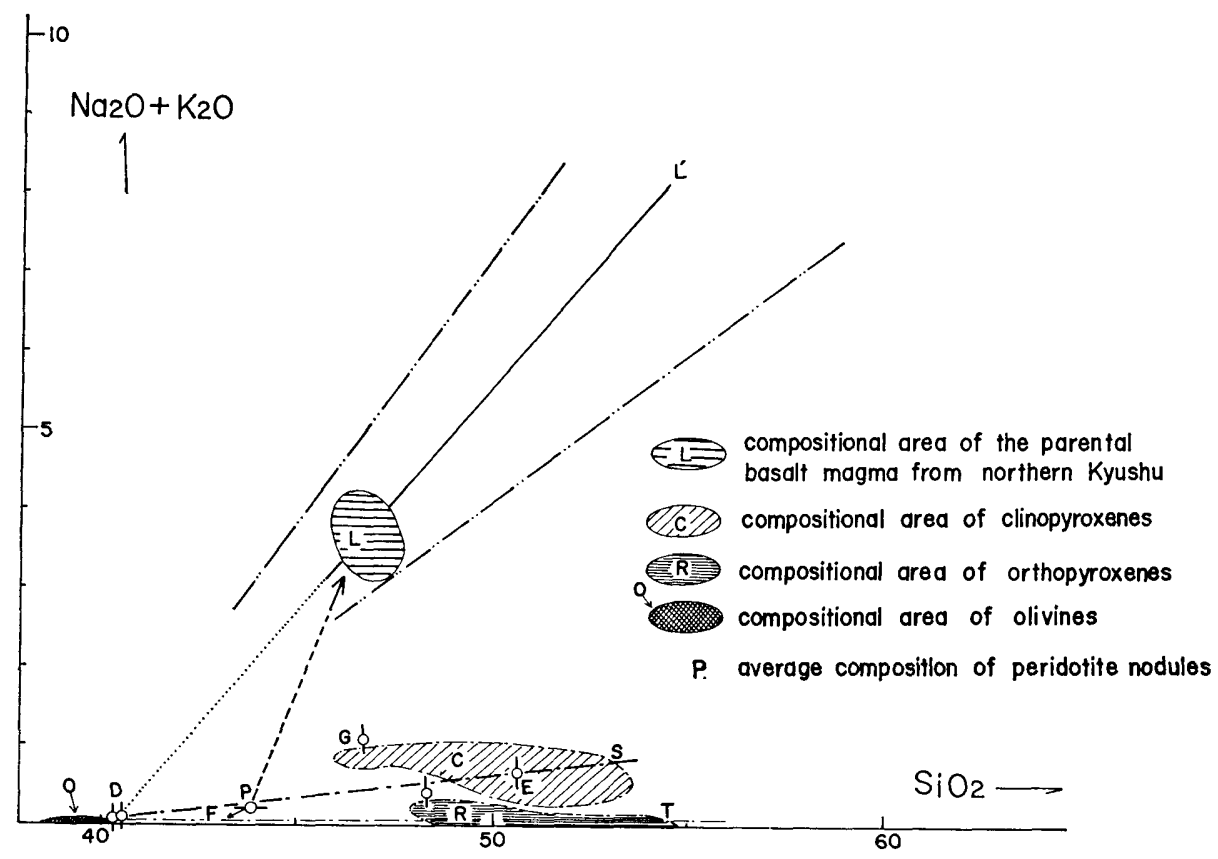

Fig. 24. Illustrative diagram for the concentration of alkalies into liquid phase during partial melting process of mantle peridotite. 
tuent minerals of basic and ultrabasic inclusions, parental basalt magmas, and phenocrysts of the inclusion-bearing basaltic host rocks is shown in Fig. 23. The Fig. 24 is reproduced from Fig. 23 for explanation. The line of (DES) is the trend line of the green type inclusion from northern Kyuhu. Point $(P)$ is the average composition of peridotite (Kushiro and Kuno, 1963). It is interesting to note that point $(P)$ is plotted approximately on the line of (DES). If chemical composition of clinopyroxene is fixed between (C) and (S) on the line of (DES), for example (E), then the ratio of olivine and clinopyroxene of the point $(P)$ is determined by the line (DPES), being counted as EP:PD $=7: 3$ (olivine 7 and clinopyroxene 3 ).

The peridotite with the composition (P) begins to melt partially forming the liquid (L), the composition of the residual peridotite tends to move from (P) to (F) and finally it reaches (F) on the line of (ODRT), and then the transfer of the alkalies into liquid phase is suspended, because the alkalies contents of initial peridotite become almost zero.

The degree of concentration of alkalies relative to silica in various kinds of rocks and minerals may be expressed in the following equation:

$$
\mathrm{C}=\frac{\mathrm{Alk}_{(a)}-\mathrm{Alk}_{(b)}}{\mathrm{SiO}_{2(a)}-\mathrm{SiO}_{2(b)}} \cdots \text { concentration factor }
$$

In Fig. 25, (C) indicates the inclination of lines (DCS) and (PL). Figure 25 shows the relation between total alkalies and silica in the parental basaltic magmas and trend lines of their differentiated products.

The concentration factor (C) of several kinds of parental basaltic magmas and their differentiated suites are shown in Table 21.

During formation of basaltic magma by partal melting, the concentration factor of the Hawaiian tholeiitic magma reaches 0.47 and that of alkali magma reaches 0.85 . The boundary line of alkalic rocks and tholeiitic rocks by magmatic crystallization from Hawaiian islands is clearly shown by MACDONALD and KATSURA (1964) and the value of $(C)$ is 0.37 . It is interesting to note that concentration factor $(C)$ in partial melting is always greater than that of fractional crystallization (Table 21).

Generally alkalies and volatile elements tend to be concentrated into liquid phase from solid phase during crystallization and during partial melting.

Variation of (C) in different rock series (Table 21) is caused by the effect of pressure and temperature on the activities of alkalies and silica in the constituent minerals of mantle peridotite.

It is conceivable that under a low pressure the activity of silica exceeds that of alkalies, and the temperature grows higher, the activity of alkalies becomes larger until it overcomes the activity of silica.

This conception is well illustrated from the zonal distribution of the assemblage of various inclusions and alkalies contents of their host rocks, which will be discussed in next paragraph. It is possible to consider that the deeper the position of parental basalt magma is produced, the larger becomes the concentration of alkalies (C).

Once the parental basalt magma is produced by partial melting, the crystal- 


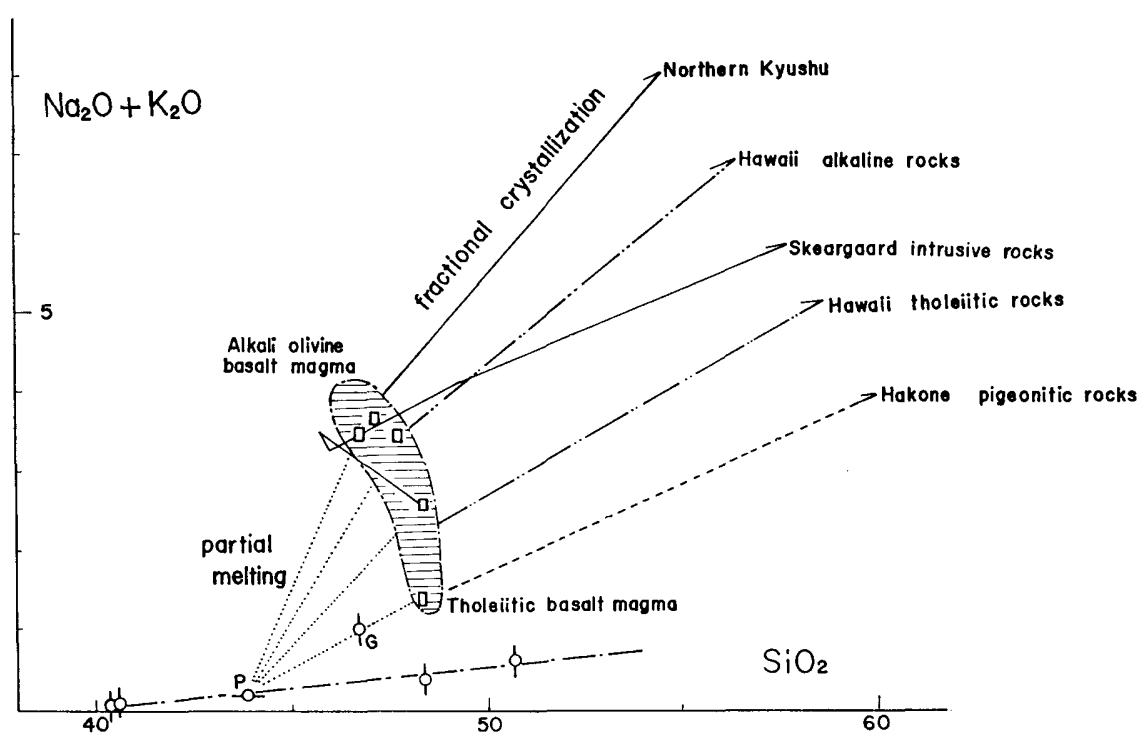

Fig. 25. Concentration of alkalies into liquid phase during partial melting process of mantle peridotite and during fractional crystallization of the basaltic rocks from several areas.

Table 21. The concentration factor of alkalies into liquid phase during partial melting and during fractional crystallization

\begin{tabular}{l|c|c}
\multicolumn{1}{c|}{ Type of rock } & partial melting & fractional crystallization \\
\hline Hakone pigeonitic rocks & 0.27 & 0.22 \\
Hawaii tholeiitic rocks & 0.47 & 0.32 \\
Skaergaard intrusive rocks & 0.55 & $0.23-0.25(?)$ \\
Hawaii alkalic rocks & 0.85 & 0.41 \\
Northern Kyushu alkali rocks & 1.15 & 0.55 \\
Boundary of alkalic rocks and & & 0.37 \\
tholeiitic rocks from Hawaiian & & \\
\hline
\end{tabular}

lization course is controled by its bulk chemical composition, temperature and pressure and also the environment of the magma reservoir. The problems would not be discussed here. The diversity of basaltic rocks may be produced by degree of fractional crystallization. However, if compositional gradient exists in the mantle peridotite, it may result the degree of partial melting of mantle peridotite.

\section{The zonal distribution of basic and ultrabasic inclusions of Cenozoic basaltic rocks in southwestern Japan}

As the Cenozoic basaltic rocks from southwestern Japan contain various kinds of basic and ultrabasic inclusions and acid xenolithes of basement rocks, it will be very interesting to investigate the geographical distribution of these inclusions and also basaltic host rocks in connection with geologic structure of the Japanese islands and adjacent areas. 
There are only brief description concerning the mode of occurrences of basic and ultrabasic inclusions and chemical relation between basatlic host rocks and their inclusions.

The detail description of basic and ultrabasic inclusions from Oki islands in the Japan Sea, Oyama and Meyama from Okayama Prefecture, Ogusoyama from Shimane Prefecture and Karatsu district from northern Kyushu were made by YAMAGUCHI (1964). The chemical differences of Cenozoic basaltic rocks of southwestern Japan were mentioned by TANEDA (1951, 1952, 1955), OJI (1961) and MAтsuмото (1961). They have demonstrated that the zonal arrangement of alkalies nature of the basaltic rocks, where the alkalies content increases from the Pacific coast through the Sea of Japan to the continental side. Kuno (1960) also mentioned that the zonal distribution of three basaltic rocks, alkali, high alumina and tholeiitic basalts were arranged almost in parallel with Japanese islands. He concluded that the distribution of these three magma types is closely related to the depth of earthquake foci. He has presented a schematic profile traversing from the Japan trench and Japanese island to the Siberian continent. In his model, he suggested that where the earthquakes are generated at shallow depth, the tholeiitic magma is produced, and as the earthquakes originate from successively deeper level, magmas are also produced at successive levels which are of high alumina basalt and alkali olivine basalt in composition (Kuno, 1966).

Figure 26 shows the geographical distribution of basic and ultrabasic inclusions, xenocrysts derived from olivine-rich inclusion and gabbros, large phenocrysts and acid xenolithes of basement rocks in the Cenozoic basaltic rocks in the region of San-in and northern Kyushu. As is clear in Fig. 26, zone A and C are almost in parallel with the western part of Japanese islands, although zone B is recognized incompletely in the surveyed area. Each zone is characterized by the following inclusions and acid xenolithes assemblage.

\begin{tabular}{lc}
\multicolumn{1}{c}{ ZONE } \\
Inclusions and acid xenolithes & $\mathrm{A}$ B C \\
Dunite & +-- \\
Cr-diopside peridotite & +-- \\
Crpyrocenite & +-- \\
Anorthite-bearing Cr-pyroxenite & + \pm- \\
Al-pyroxenite & ++- \\
Olivine gabbro & ++- \\
Olivine-free gabbro & + \pm- \\
Xenocrystic Al-augite and Al-hypersthene & +++ \\
Xenolithes of acid basement rocks & +++
\end{tabular}

In other words the zone $\mathrm{C}$ is characterized by the xenocrystic Al-augite and Al-hypersthene (large phenocryst), and xenolithes of acid rocks. The zone B is characterized by the black type inclusions and acid xenolithes. Abundant green type inclusions and less abundant black type inclusions, and acid xenolithes of basement rocks are found in the zone $A$. 


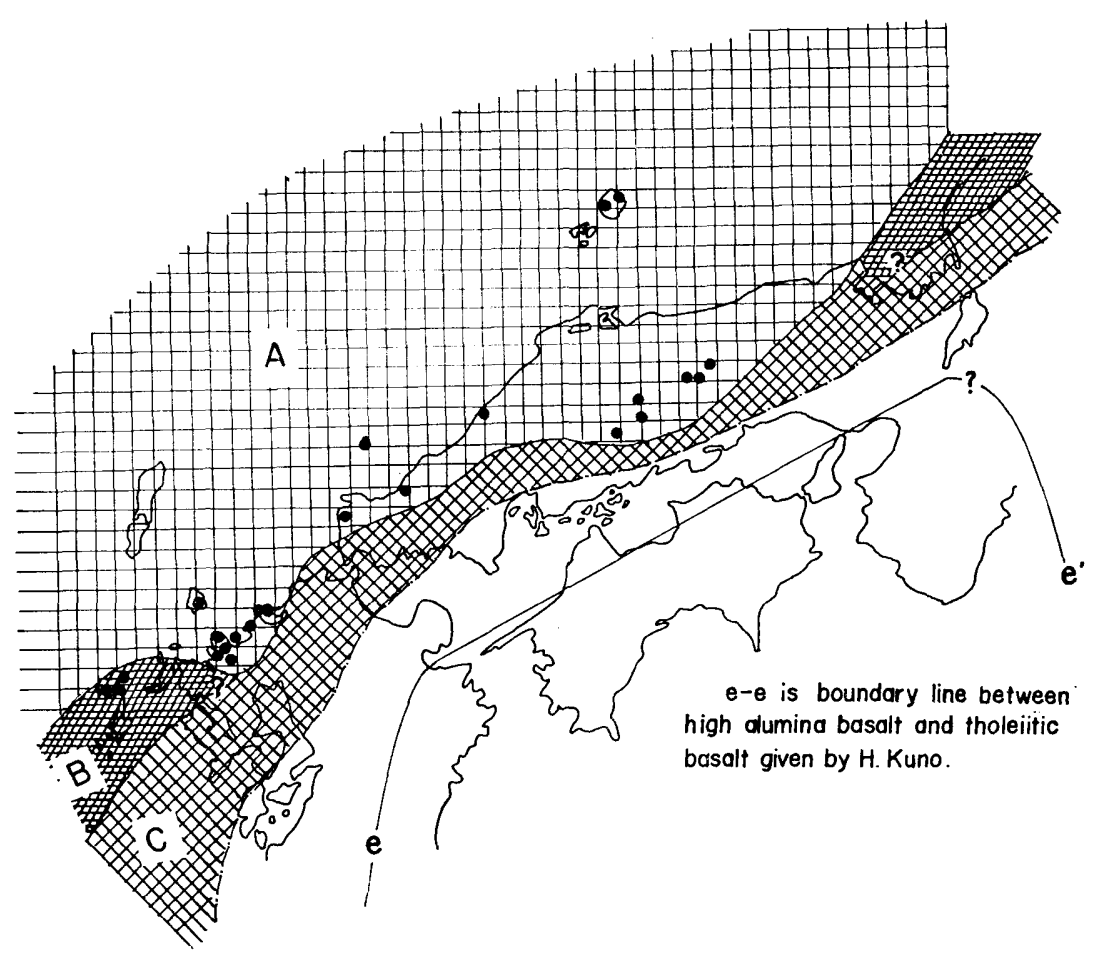

Fig. 26. Zonal distribution of basic and ultrabasic inclusions in the Cenozoic basaltic rocks of southwestern Japan.

A: Zone of green type inclusion.

(Basalts of this zone have both green type and black type inclusions, and xenocrysts derived from black type inclusion)

B: Zone of black type inclusion.

(Basalts of this zone have black type inclusion and xenocrysts derived from black type inclusion)

C: Zone of xenocrysts minerals.

(Basalts of this zone have no inclusions but xenocryst minerals only, which are derived from black type inclusions)

I should like to discuss below the meaning of this characteristic distribution.

The stability relation of eclogite and gabbro is examined experimentally by RINGWOoD and GREen $(1966,1967)$. They concluded that eclogite appears to be more stable at the lower level of the continental crust. Recently, however, pyroxene-spinel symplectite has been found in the olivine gabbro at Ichinomegata, Akita Prefecture by KUNo (1967) and Iki island, Nagasaki Prefecture by AoKI (1968).

The experimental data of the minerals assemblage (i.e. symplectite) suggest that anorthite and forsterite $(1: 1 \mathrm{~mol} . \%)$ change to clinopyroxene, orthopyroxene, anorthite and spinel at $9 \mathrm{~Kb} .1300^{\circ} \mathrm{C}$ and also at $8 \mathrm{~Kb} .1100^{\circ} \mathrm{C}$ (Kushiro and YoDER, 1966). The eclogite has never been found in the area studied in the present paper, whereas gabbro inclusions is found abundantly in the Cenozoic basaltic rocks in southwestern Japan. Also, I have never found pyroxene-spinel symplectite in gabbro and Al-pyroxenite. 
Absence of symplectite in these inclusions suggests that the pressure is insufficient for transformation from olivine-plagioclase assemblage to pyroxenespinel symplectite. From this fact, it can be concluded that the black type inclusions may originate at position shallower than $30 \mathrm{~km}$ in depth. On the other hand, the green type inclusions ( $\mathrm{Di}+\mathrm{En}+\mathrm{Fo}+\mathrm{Spi}$ and $\mathrm{Di}+\mathrm{En}+\mathrm{An}+\mathrm{Spi}$ ) is stable between the stability field of gabbro and garnet peridotite (KUSHIRO and YoDER, 1966; RINGWood and GREeN, 1967, 1968).

The zonal arrangement of inclusions assemblage and experimental data suggest that the positions where the basaltic magmas are produced probably deepen from the side of the Setouchi Inland Sea and under the Japanese islands to East Asiatic continent (Siberia), because the xenolithes and inclusions around the Sea of Japan have characters which indicate a deeper origin than those closer to the Setouchi Inland Sea (Fig. 1 and Fig. 26).

The world-wide distribution of basic and ultrabasic inclusion is discussed by FORBES and KUNo (1967). They paid much attention to the comparative petrology of basaltic host rocks and their inclusions and regional distribution of the inclusions. According to their conclusions, both continental and oceanic basalt magmas are derived from the upper mantle and the origin of peridotite inclusions is related to the genesis of basaltic magmas in these zone.

As already stated, the two distinct assemblages of inclusions, such as, the green type and the black type inclusions, the former is probably derived from the mantle and the latter is formed by the accumulation of crystals in magma reservoir shallower than $30 \mathrm{~km}$ in depth.

\section{Summary of results}

The following is a summary of result of the present study of basic and ultrabasic inclusions in the basaltic rocks in the northern Kyushu and San-in districts.

(1) The basic and ultrabasic inclusions can be divided into two groups as follow:

i) The green type inclusion

ii) The black type inclusion

(2) Both oxide and silicate minerals of the green type inclusions are characterized by a large amount of $\mathrm{Cr}_{2} \mathrm{O}_{3}$, while those of black type inclusions are characterized by a large amount of $\mathrm{Al}_{2} \mathrm{O}_{3}$.

(3) The mode of occurrence shows that the green type inclusions may originate from the upper mantle. The textural, optical and chemical characters of constituent minerals seem to show that they are of deeper origin than that of black type inclusions.

(4) The black type inclusions are formed by accumulation of crystals, crystallized from basaltic magma in the reservoir shallower than $30 \mathrm{~km}$ in depth.

(5) In the basaltic rocks near the coast of the Japan Sea there are both green type and black type inclusions, whereas the basaltic rocks of the Setouchi district have only the black type inclusions and acid xenolithes of basement rocks. 
The inclusions assemblage is closely related to the chemical composition of the basaltic host rock. Namely, alkali basalt has both green type and black type inclusions as seen in the basaltic rocks around the Japan Sea, whereas alkali poor basalt has inclusions of black type only, as seen in the basaltic rock from the area closer to Setouchi Inland Sea district.

\section{References}

AoKI, K. (1949) : Petrology of alkali rocks of the Iki Island and Higashi-matsuura District, Japan. Sci. Rept. Tohoku Univ., Ser. 3, 6, 261-310.

(1960): Differentiation of quartz dolerite dyke of the hypersthenic rock series, Kabeshima Island, Saga Prefecture. Jour. Jap. Assoc. Min. Petrol. Econ. Geol., 44, 47-53.

(1964): Clinopyroxenes from alkaline rocks of Japan. Am. Mineral., 49, 1199-1223.

(1966): Phenocrystic spinel-magnetite solid solution in trachyandesite from Iki Island. Jour. Jap. Assoc. Min. Petrol. Econ. Geol., 55, 127-128.

(1968): Petrology of ultrabasic and basic inclusions in alkali basalts, Iki Island, Japan. Am. Mineral., 53, 241-256.

Aoyama, M. (1942): Large augite crystals in basalt from Takashima, Karatsu. Jour. Geol. Soc. Jap., 49, 206-207.

Atras, L. (1952): The polymorphism of $\mathrm{MgSiO}_{3}$ and solid state equilibria in the system $\mathrm{MgSiO}_{3}-\mathrm{CaMgSi}_{2} \mathrm{O}_{6}$. Jour. Geol., 60, 125-147.

Bailey, E. B., Clough, C. T., Wright, W. B., Richey, J. E. and Wilson, G. V. (1924) : The Tertiary and post-Tertiary geology of Mull, Loch Aline and Oban. Mem. Geol. Surv. U. K., 13-39.

Banno, S., Kushiro, I. and Matsui, Y. (1963): Notes on rock-forming minerals: Enstatite from a garnet peridotite inclusion in kimberite. Jour. Geol. Soc. Jap., 69, 157-159.

Barth, Tom. F. W. (1936): The crystallization process of basalt. Am. Jour. Sci., $31,321-351$.

Benson, W. N. (1941): Caniozoic petrographic provinces in New Zealand and their residual Magma. Am. Jour. Sci., 239, 537-552.

Beloussov, V. V. and Ruditch, E. M. (1961): Island Ares in the Development of the Earth's Structure (Especially in the region of Japan and Sea of Okhotsk). Jour. Geol., 69, 647-658.

BoYd, F. R. and ENGLAND, J. L. (1958): Melting of diopside under high pressure. Carnegie Inst. Washington, Year Book, 57, 83-87.

and - (1960): Mineral of the Mantle. Carnegie Inst. Washington, Year Book, 50, 47-52.

Bowen, N. L. (1958): The Evolution of Igneous rocks. Princeton Univ. Press, 1-332. and Schairer, J. F. (1935): The system $\mathrm{MgO}-\mathrm{FeO}-\mathrm{SiO}_{2}$. Am. Jour. Sci., 29, 151-217.

Brown, G. M. (1957) : Pyroxene from the early and middle stage of fractionation of Skeargaart intrusion, East Greenland. Min. Mag., 31, 511-543.

(1960): Effect of Ion substitution on the Cell Dimension of the common clinopyroxene. Am. Mineral., 45, 18-38.

and Vincent, E. A. (1962): Pyroxenes from the late stage of fractionation of Skeargaard intrusion, East Greenland. Min. Mag., 31, 511-543.

Buddington, A. F. (1943): Some petrological concepts and interior of the earth. Am. Mineral., 28, 119-140.

Chapman, R. W. and Williams, C. R. (1935): Evolution of the White Mountain Magma series. Am. Mineral., 20, 502-530.

Clark, S. P., Schairer, J. F. and Neufville, J. (1962): Phase relation in the system 
$\mathrm{CaMgSi}_{2} \mathrm{O}_{8}-\mathrm{CaAl}_{2} \mathrm{SiO}_{8}-\mathrm{SiO}_{2}$ at low and high pressure. Carnegie Inst. Washington, Year Book, 61, 59-68.

Dallwitz, W. B., Green, D. H. and Tompson, J. E. (1966): Clinoenstatite in the volcanic rock from the Cape Vogel Area, Papua. Jour. Petrol., 7, 375-403.

DeEr, W. A., Howie, R. A. and Zussman, A. (1963) : Rockforming minerals, 2, 1-379, Longman.

Deriu, M. (1959) : Olivinie e pirosseni di noduli in basalti della Sardegna centrooccidentale, Period. Mineral., 28, 259-283.

Eaton, J. P. and Murata, K. J. (1961) : How volcanoes grow, Science, 132, 925-938.

ERNst, T. (1936) : Der Melilith-Basalt des Westberges bie Hofgeismar, Nordlich von Kassel, ein Assimlations-produkt Ultrabasischer Gestine., Chemi der Erde, 10, 631-666.

Fairbairn, H. W. (1943) : Packing ionic minerals. Bull. Geol. Soc. Am., 54, 13051374.

Forbes, R. B. and Kuno, H. (1967) : Peridotite inclusion and basaltic host rock. Ultramafic and related rocks. edt. by P. J. WYLLIE, Wiley 1-446.

FukuYama, K. (1961): Drusy pigeonite in the olivine basalt from Senryu-Shiratake hill, Nagasaki Prefecture, Japan. Fac. Sci. Kumamoto Univ., Geology, 4, 18-24.

Gilluly, J. (1937): The water content of magmas. Am. Jour. Sci., 33, 430-441.

GoraI, M. (1951): On the origin of the diversity of igneous rocks. Jour. Geol. Soc. Jap., 57, 387-397.

GreEN, G. H. (1963) : Alumina content of enstatite in Venezuelan high-temperature peridotite. Bull. Geol. Soc. Am., 74, 1394-1402.

(1964): The petrogenesis of the high-temperature peridotite in the Lizard area, Conwall. Jour. Petrol., 5, 134-188.

and RiNgwood, A. E. (1967): An experimental investigation of the gabbro to to eclogite transformation and its petrological application. Geosch. et Cosm. Acta, 31, No. 5, 767-834.

HAMAD, S. D. (1962): The chemistry and mineralogy of olivine nodules of Calton Hill Derbyshire. Min. Mag., 260, 491-497.

HARRIS, P. G. and Rowell, J. A. (1960): Some geochemical aspects of Mohorovicic discontinuity. Jour. Geophys. Res., 65, 2443-2459.

HaRumoto, A. (1951): Limburgite from Sukumozuka in the vicinity of Tuyama, Okayama Prefecture. Jour. Geol. Soc. Jap., 57, 318.

(1952): Melilite-nepheline basalt, its olivine nodule and other inclusions from Nagahama, Japan. Mem. Coll. Sci., Kyoto Univ., Ser. B, 20, 69-88.

Hess, H. H . (1938) : A primary peridotite magma. Am. Jour. Sci., 35, 321-344.

(1941): pyroxene of common maffic magmas: Part 2. Am. Mineral., 26, $537-594$.

and Henderson, E. P. (1949): The Moor County mteorite; A further study with comet on it primedial environment. Am. Mineral., 34, 494-507.

(1949) : Chemical composition and optical properties of common clinopyroxene. Am. Mineral., 34, 621-666.

(1952): Orthopyroxene of the Bushveld type, ion substitution and changes in unite cell dimensions. Am. Jour. Sci., Bowen Vol., 173-187.

(1960): Stillwater Igneous complex, Montana. Geol. Soc. Am., Memore 80, $1-227$.

Hillebrand, W. F. and Lundell, G. E. F. (1929): Applied inorganic Analysis. John Wiley and Sons Inc., New York.

IsHIBASHI, K. (1962): Alkali-pyroxene in the doleritic basalt flow from the HigashiMatsuura district, Saga Prefecture. Jour. Jap. Assoc. Petrol. Econ. Geol., 47, 213-222.

(1964): Large phenocrystic pyroxenes in basaltic rocks of Northern Kyushu, Japan. Sci. Rept. Kyushu Univ., 7, 47-56.

IWASAKI, I. (1941): Chemical analysis of silicate. Iwanami Shoten.

- and Katsura, T. (1950): The chemical composition of magnetite from vol- 
canic rocks, and special references of magnetic stage of the host rocks. Jour. Mining Geol. Kyushu, 18, 197-203, 256-259, 291-295.

Iwasaki, I., Katsura, T., Yoshida, M. and Tarutani, T. (1957) : Rapid analysis of magnetite and titanomagnetite. Anal. Chem., 6, 211-215.

and IWASAKI, B. (1962): Geochemical investigations of Volcanoes in Japan; Vanadium content of the Lava Flow in 1950-1951 from Volcano Oshima, Izu, Japan. Bull. Chem. Soc. Japan., 35, 454-459.

JACKSON, E. D. (1961): Primru texture and mineral associations in the Ultramaffic zone of the Stillwater Complex, Montana. U. S. Geol. Surv. Profess. Paper, No. 358 .

JAMBOR, J. L. and SMith, C. H. (1964): Olivine composition determination with small diameter X-ray camera. Min. Mag., 33, 730-741.

Katsura, T. and Kushiro, I. (1961): Titanomagnetite in igneous rocks. $A m$. Mineral., 46, 134-145.

Katsura, T. and MUAN, A. (1964) : : Equilibria in the system $\mathrm{FeO}-\mathrm{Fe}_{2} \mathrm{O}_{3}-\mathrm{Cr}_{2} \mathrm{O}_{3}$ at $1300^{\circ} \mathrm{C}$. Trans. AIME, 230, 77-84.

Katsura, T. (1967) : Pele's hair as a liquid of Hawaiian tholeiitic basalts. Geochem. Soci. Jap. Bull., 1, 157-168.

and SHibATA, K. (1967): On the course of crystallization of basaltic magma in various oxygen partial pressure. Summary of discussion of geochem. Soc. Japan.

KENNEDY, W. Q. (1933) : Trend of differentiation in basaltic magmas. Am. Jour. Sci., 25, 239-256.

_ and Anderson, E. M. (1938) : Crystal layers and the origin of magmas. Bull. Volcano, 3, 24-82.

Kennedy, G. C. (1955): Some aspects of the role of water in rock melts. Geol. Soc. Am. Special paper 62, 489-504.

KERTz, R. (1959) : Chemical study of garnet, biotite and hornblende from gneiss of Southwestern Qubec with emphasis on distribution of element in coexisting minerals. Jour. Geol., 67, 371-402.

(1961): Some application of thermodynamics to co-existing minerals of variable composition, Example: orthopyroxene-clinopyroxene and orthopyroxenegarnet. Jour. Geol., 69, 361-387.

KitahaRA, J. (1954): On the chromite from the Hiroosa Mine, Shizuoka Prefecture. Jour. Jap. Assoc. Min. Petrol. Econ. Geol., 26, 515-535, 573-594.

Kuno, H. (1941) : Pyroxenes of common maffic magmas; part 1 and 2. Am. Mineral., 26, 515-535, 573-594.

(1950): Petrology of Hakone Volcano and adjacent areas. Bull. Geol. Soc. $A m ., 61,957-1019$.

and Nagashima, K. (1952): Chemical composition of hypersthene and pigeonite in equilibrium in magma. Am. Mineral., 37, 1000-1006.

and Hess, H. H. (1953): Unite cell dimensions of clinoenstatite in relation to other common clinopyroxenes. Am. Jour. Sci., 251, 741-752.

(1954): Study of orthopyroxenes from volcanic rocks. Am. Mineral., 39, 30-46.

(1955): Ion substitution in the diopside-pigeonite series of clinopyroxenes. Am. Mineral., 40, 70-93.

, Yamasaki, K., IIDA, C. and NAgashima, K. (1957) :: Differentiation of Hawaiian magmas. Jap. Jour. Geol. Geogr., 28, 179-218.

(1960) : High-alumina basalt. Jour. Petrol., 1, 121-145.

(1964): Aluminian augite and bronzite in alkali basalt from Takashima, North Kyushu, Japan. Advance Front in Geol. and Geophy., 205-220.

(1966): Lateral variation of basalt magma type across continental margins and island arcs. Bull. Volcano., 29, 195-222.

(1967): Maffic and ultramaffic nodules from Itinomegata, Japan. Ultramafic and related rcoks, edt. WYLLIE, P. J., Wiley 1-446. 
Kurasawa, H. (1967) : Petrology of Kita-Matsuura basalts in the northwest Kyushu, southwestern Japan. Rept. Geol. Surv. Jap., No. 217.

Kushiro, I. (1959) : Preliminary note on alkali-dolerite from Atumi district, Northern Japan. Jap. Jour. Geol. Geogr., 30, 259-272.

(1960): Si-Al relation in clinopyroxenes from igneous rocks. Am. Jour. Sci., $258,548-554$.

(1962): Clinopyroxene solid solutions part 1, the $\mathrm{CaAl}_{2} \mathrm{SiO}_{6}$ component. Jap. Jour. 33, 213-220.

- and KUNo, H. (1963) : Origin of primary basalt magma and classification of basaltic rocks. Jour. Petrol., 4, 75-89.

- and YoDER, H. S. Jr. (1966): Anorthite-Forsterite and Anorthite-Enstatite reacitons and their bearing on the Basalt-Eclogite transformation. Jour. Petrol., 7, 337-362.

LACrorx, A. (1932): La Constitution lithologique des iles volcanoques de la Polynesie Australe. Mem. Acad. Sci. Paris, 58.

Le BAss, M. J. (1962) : The role of aluminum in igneous clinopyroxene with relation to parantage. Am. Jour. Sci., 260, 267-288.

LeAKe, B. E. (1964) : New light on the Dawros peridotite, Connemara, Iceland. Geol. Mag., 101, 62-75.

MACKENZIE, D. B. (1960): High temperature alpine type peridotite from Venezuella. Bull. Geol. Soc. Am., 71, 308-318.

MaCDOUGALL, I. (1961): Optical and chemical studies of pyroxenes in a differentiated Tasmania dolerite. Am. Mineral., 46, 661-687.

MACDonald, G. A. (1960): Dissimilarity of continental and oceanic rock types. Jour. Petrol., 1, 172-177.

and Katsura, T. (1961):: Variation in the Lava of the 1959 Eruption in Kilauea Iki. Pacific Sci., 15, 358-368.

and - (1962): Relationship of petrographic studies in Hawaii. Crust Pacific Basin, Geophy. Monogr., No. 6, 187-195.

and - (1964): Chemical composition of Hawaiian lavas. Jour. Petrol., $5,82-133$.

MIYAKE, T. (1948) : Revision of analysis of augite from Nishigatake. Mineral. Geol., $8,77-80$.

Matsushita, H. (1966): Geologic Structure of the Karatsu Coal-field with special Reference to the Basaltic lava Chambers. Memorial Vol. Prof. Susumu MatsuSHITA, 75-82.

Matsumoto, Y. (1961): Petrological studies on Matsuura basalt from northern Kyushu, Japan. Rept. Res. Ind. Kyushu Univ., No. 30, 1-99. (1963): The Late Cenozoic Volcanism in the Northern and Central Kyushu, Japan. Rept. Res. Ind. Kyushu Univ., No. 34, 1-20.

MiYAShiro, A. (1965): Metamorphic rocks and Metamorphic Belt. 1-458, Maruzen Co., Tokyo.

Mlmer, C. G. and White, W. B. (1966) : Existence of Chromaus Ion in the spinel solid solution series $\mathrm{FeCr}_{2} \mathrm{O}_{4}-\mathrm{MgCr}_{2} \mathrm{O}_{4}$. Jour. Am. Ceram. Soc., 49, 51-52.

Muir, I. D. (1954) : Crystallization of pyroxene in an iron-rich diabase from Minesota. Min. Mag., 20, 377-388.

- Tilley, C. E. and Scoon, J. D. (1957) : Contributions to the petrology of Hawaiian basalts. Am. Jour. Sci., 255, 241-253.

Murrary, R. J. (1954): The clinopyroxenes of the Garbh Eilean sill, Shiant Inlets. Geol. Mag., 61, 17-31.

Mueller, R. F. (1960): Compositional characteristics and equilibrium relations in mineral assemblage of metamorphosed iron formation. Am. Jour. Sci., 258, $449-497$.

Nixson, P. H., KNoRring, O. V. and Rooke, J. M. (1963): Kimberlite and associated inclusions of Basutoland; A mineralogical and chemical study. Am. Mineral., $48,1090-1132$. 
Norton, D. A. and Cleavan, W. S. (1959): The optical mineralogy, chemistry and X-ray crystallography of ten clinopyroxenes from Pensylvenia and Delaware Piedomont Province. Am. Mineral., 44, 844-874.

O'HARA, M. J. (1963) : Distribution of Iron between co-existing olivines and calcium poor pyroxenes in peridotite, gabbro and other magnesium environments. $A m$. Jour. Sci., 261, 32-46.

and Mercy, L. P. (1966) : Eclogite, peridotite and pyrope from Navajo County, Arizona and New Mexico. Am. Mineral., 51, 336-352.

OJI, Y. (1961a): On the augite phenocryst in alkali basalts from the Abu district. Jour. Jap. Assoc. Mineral. Petr. Econ. Geol., 45, 66-70.

(1961b) : Olivine in the alkali basalts of western San-in and northern Kyushu. Jour. Jap. Assoc. Mineral. Petr. Econ. Geol., 45, 133-136.

(1961c): Petrology of the Cenozoic basaltic rocks of western San-in and Northern Kyushu, Japan. Bull. Fukuoka Univ. Education, special Vol. No.1, $1-89$.

ONUki, H. (1963): Petrology of the Hayachine Ultramafic complex in the Kitakami Mountainland, Northern Japan. Sci. Rept. Tohoku Univ., Ser. 3, 8, 421-295. and Tiba, T. (1964): Petrochemistry of the Ichinohe alkali plutonic complex, Kitakami Mountainland, Northern Japan. Sci. Rept. Tohoku Univ., Ser. 3, 9, 123-153.

(1965): Petrochemical research on the Horoman and Miyamori ultramafic intrusives, Northern Japan. Sci. Rept. Tohoku Univ., Ser. 3, 9, 217-276.

PECK, K. C. (1964) : Systematic analysis of silicate. Bull. U. S. Geol. Surv., 1170, 89.

PoldervaArt, A. (1947) : The relationship of orthopyroxene to pigeonite. Min. Mag., 28, $164-172$.

Powers, H. A. (1955): Composition and origin of basaltic magma of the Hawaiian Islands. Geochim. et Cosm. Acta, 7, 77-107.

RAMBerg, H. and De Vore, G. (1951): The distribution of $\mathrm{Fe}$ and $\mathrm{Mg}$ in coexisting olivine and pyroxenes. Jour. Geol., 59, 193-210.

RINGwood, A. E. (1956) : Melting relationship of Ni-Mg olivines and some geochemical implications. Geochim. et Cosm. Acta, 10, 297-303.

- and Green, D. H. (1966): An experimental investigation of the GabbroEclogite transformation and some geophysical implication. Tectonophysic, 3, $383-427$.

Ross, C. E., Foster, M. D. and MYers, A. T. (1954) : Origin of dunite and of olivinerich inclusions in basaltic rocks. Am. Mineral., 39, 639-737.

Ruchmick, J. C. and Noвle, J. A. (1959): Origin of ultramafic complex at Union Bay, Southeastern Alaska. Bull. Geol. Soc. Am., 70, 981-1017.

SANDELL, E. B. (1950): Colorimetric Determination of Trace of Metals. Interscience Pub. Inc.

SeArle, E. J. (1960): Petrochemistry of the Auckland basalt. N. Z. Jour. Geol. Geophys., 3, 23-40.

Shapiro, L. and Brannock, W. W. (1962): A Rapid analysis of silicate, carbonate and phosphate rocks. Bull. U. S. Geol. Surv., 1144-A.

Stevens, R. E. (1944) : Composition of some chromites of the Western Hemisphere. Am. Mineral., 29, 1-34.

SUGI, K. (1942): Petrological studies on the basaltic rocks from San-in and northern Kyushu, southwestern Japan. Mem. Fac. Sci. Kyushu Univ., Ser. D, 1, 69-90.

SugrmurA, A. (1959): Geographiche Verteilung der Charakterstendezen des Magmas in Japan. Bull. Volcano. Soc. Jap., Ser. 2, 4, 77-103.

TANedA, S. (1951): Studies on Volcanoes in Japan; The Chemical Composition of the Lavas. Sci. Rept. Kyushu Univ., Ser. D, 3, 55-76. (1952) : New average chemical composition of Japanese effusive rocks. Jour. Geol. Soc. Jap., 58, 517-522.

(1962): Frequency Distribution and Average Chemical Composition of the Volcanic Rocks in Japan. Mem. Fac. Sci. Kyushu Univ., Ser. D, 12, 237-255. 
(1965): Areal rock character in Japan. Sci. Rept. Kyushu Univ., Ser. D, 8, $1-40$.

Talbote, J. L., Hobbs, B. E., Wilshire, H. G. and Sweatman, T. R. (1963) : Xenolithes and xenocrysts from lavas of the Kergulen Archipelago. Am. Mineral., $48,159-179$.

ThAYER, T. P. (1964) : Preliminary chemical correlation of chromite with the containing rocks. Econ. Geol., 41, 202-217.

TiBA, T. (1966) : P'etrology of the alkaline rocks of the Takakusayama district, Japan. Sci. Rept. Tohoku Univ., Ser. B, 9, 541-610.

Tomita, T. (1936): Geology of Dogo, Oki island, in the Japan Sea. Jour. Shanghai Sci. Inst., Ser. 2, 37-146.

(1951): Type of magmatic evolution. Sci. Rept. Kyushu Univ., Ser. D, 3, 77-104.

TURNER, F. J. and VERHOogen, J. (1951): Igneous and Metamorphic petrology. Mcgraw-Hill Inc., New York.

WAGER, L. R. and DEER, W. A. (1939) : Geological investigations in East Greenland; part 3 , the petrology of the Skeargaard intrusion, Kangerdlugssuak. Medd. Greenland, 105, 1-352.

WARSHAW, I. and KeITH, M. L. (1954): Solid solution and chromium oxide loss in part of the system $\mathrm{MgO}-\mathrm{Al}_{2} \mathrm{O}_{3}-\mathrm{Cr}_{2} \mathrm{O}_{3}-\mathrm{SiO}_{2}$. Jour. Am. Ceram. Soc., 37, 161.

Washington, H. H. (1930): The chemical analysis of rocks. John Wiley and Sons Inc.

White, A. J. R. (1964): Cliopyroxenes from eclogite and basic granulites. $A m$. Mineral., 49, 883-888.

Whiте, R. W. (1966) : Ultramafic inclusions in basaltic rocks from Hawaii. Contr. Mineral. Petrol., 12, 245-314.

WiLkinson, J. F. G. (1956): Clinopyroxene of alkali olivine basalt magmas. $A m$ Mineral., 41, 724-743.

Wilshire, H. G. and Binns, R. H. (1961): Basic and ultrabasic xenolithes from volcanic rocks of New South Wales. Jour. Petrol., 2, 185-208.

WiNCHEL, A. N. (1951) : Element of optical mineralogy. Chapman and Hall Inc.

YAGI, K. (1958) : Petrochemistry of Cenozoic alkalic rocks of Japan and surrounding area. Bull. Volc. Soc. Jap., Ser. 2, 3, 63-75.

Yamaguchi, M. (1958): Petrography of the Otozan Flow on Shodo-shima island, Serouchi Inland Sea, Japan. Mem. Fac. Sci. Kyushu Univ., Ser. D, 6, 217-238. (1961): Chrome-diopside in Horoman and Higashiakaishi Peridotite, Japan. Mem. Fac. Sci. Kyushu Univ., Ser. D, 10, 233-245. (1964): Petrogenic significances of ultrabasic inclusions in basaltic rocks from Southwest Japan. Mem. Fac. Sci. Kyushu Univ., Ser. D, 15, 163-219.

Yoder, H. S. Jr. and TIlley, C. E. (1961) : Simple basalt system. Carnegie Inst. Washington, Year Book, 60, 106-113.

Yoder, H. S. Jr. and TrLLEY, C. E. (1962) : Origin of basalt magma; An experimental study of natural and synthetic rock systems. Jour. Petrol., 3, 342-532. 
Kiyoshi ISHIBASHI

Petrochemical Study of Basic and Ultrabasic Inclusions in Basaltic rocks from Northern Kyushu, Japan

Plates $18 \sim 25$ 
Plate 18 


\section{Explanation of Plate 18}

Fig. 1. Large phenocrysts of aluminous augite $(\mathrm{Au})$ and labradolite $(\mathrm{Pl})$ in the basaltic rock from Takashima. Gabbro $(\mathrm{Ga})$ and peridotite $(\mathrm{Pe})$ inclusions are also contained.

Fig. 2. Roughly separated phenocrysts of aluminous augite and aluminous hypersthene in the scoriaceous rock from Takashima, showing euhedral outline. 

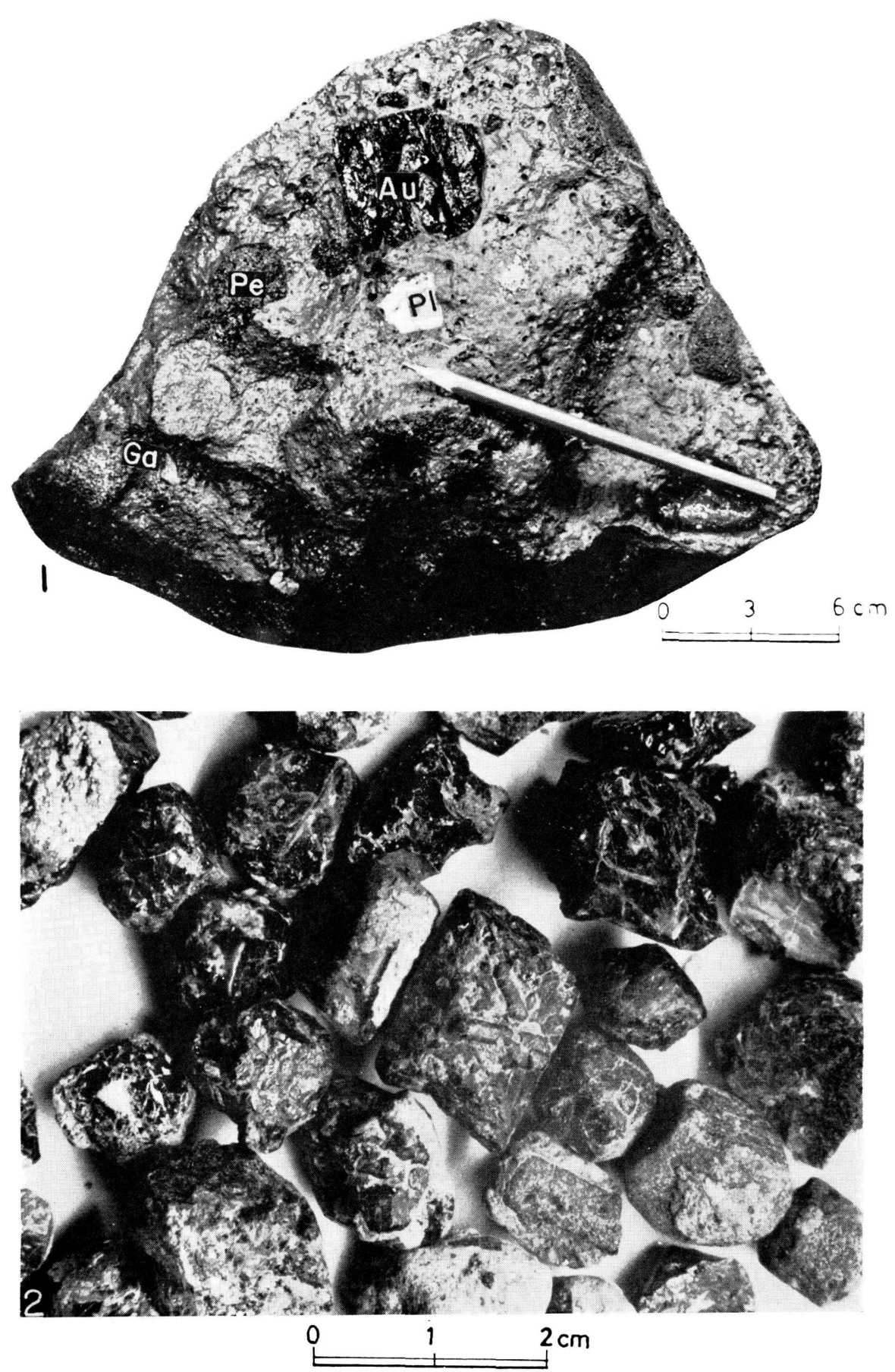

K. IsHibashi : Basic and Ultrabasic Inclusions 
Plate 19 


\section{Explanation of Plate 19}

Fig. 3. Chromian diopside (Di) and plagioclase ( $\mathrm{Pl}$ ) showing banded texture, in the anorthite-bearing Cr-pyroxenite.

Fig. 4. Banded gabbro, consisting aluminous augite $(\mathrm{Au})$, aluminous hypersthene $(\mathrm{Hy})$ and plagioclase $(\mathrm{Pl})$ from Kurose. 

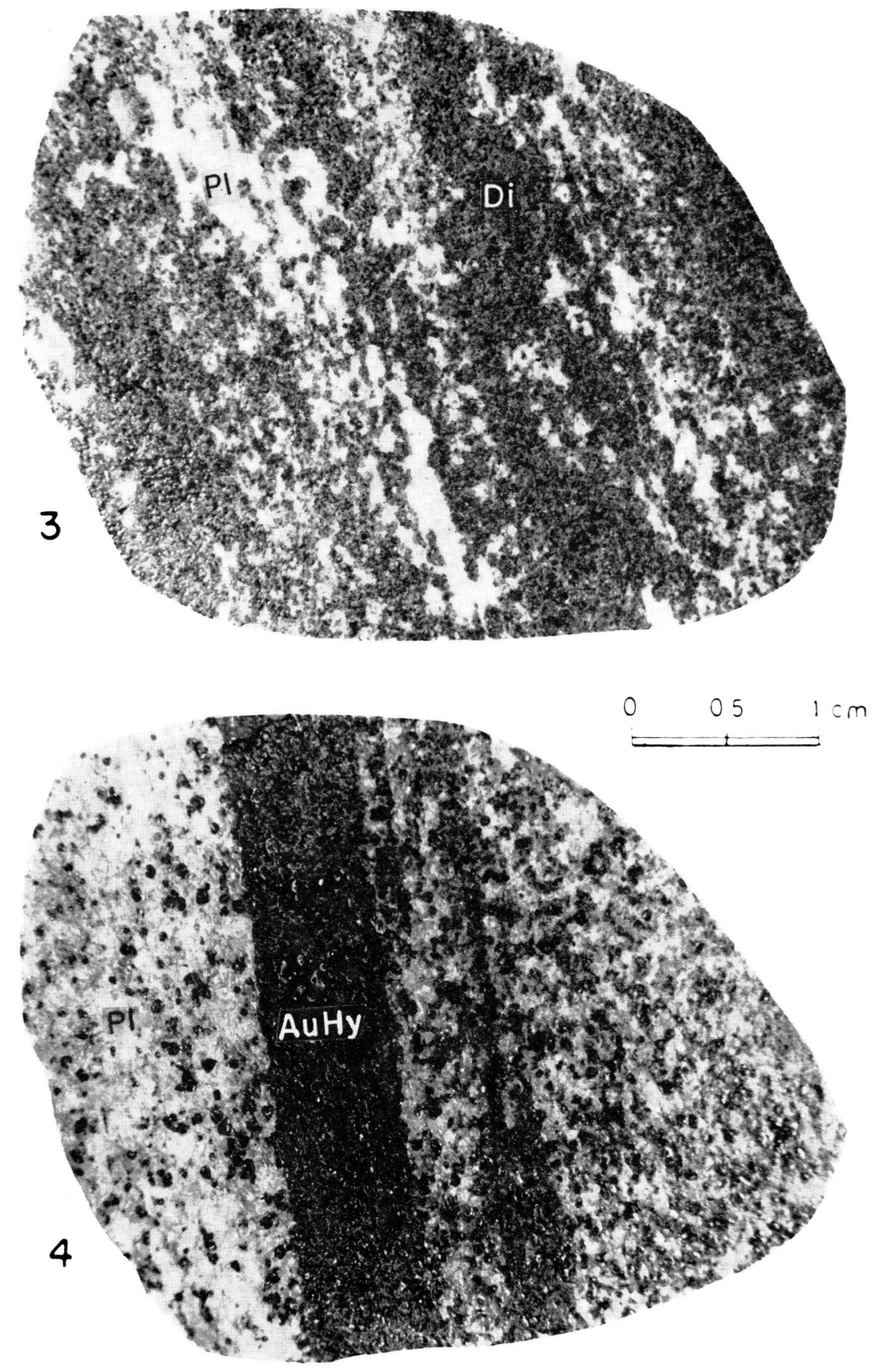

K. Ishibashi: Basic and Ultrabasic Inclusions 
Plate 20 


\section{Explanation of Plate 20}

Fig. 5. Photomicrograph showing a contact of dunite $(\mathrm{Du})$ and basalt $(\mathrm{Ba})$. The dunite consists of olivine (Ol) and chromite (Chr) open niclos.

Fig. 6. Crossed nicols of Fig. 5 . 


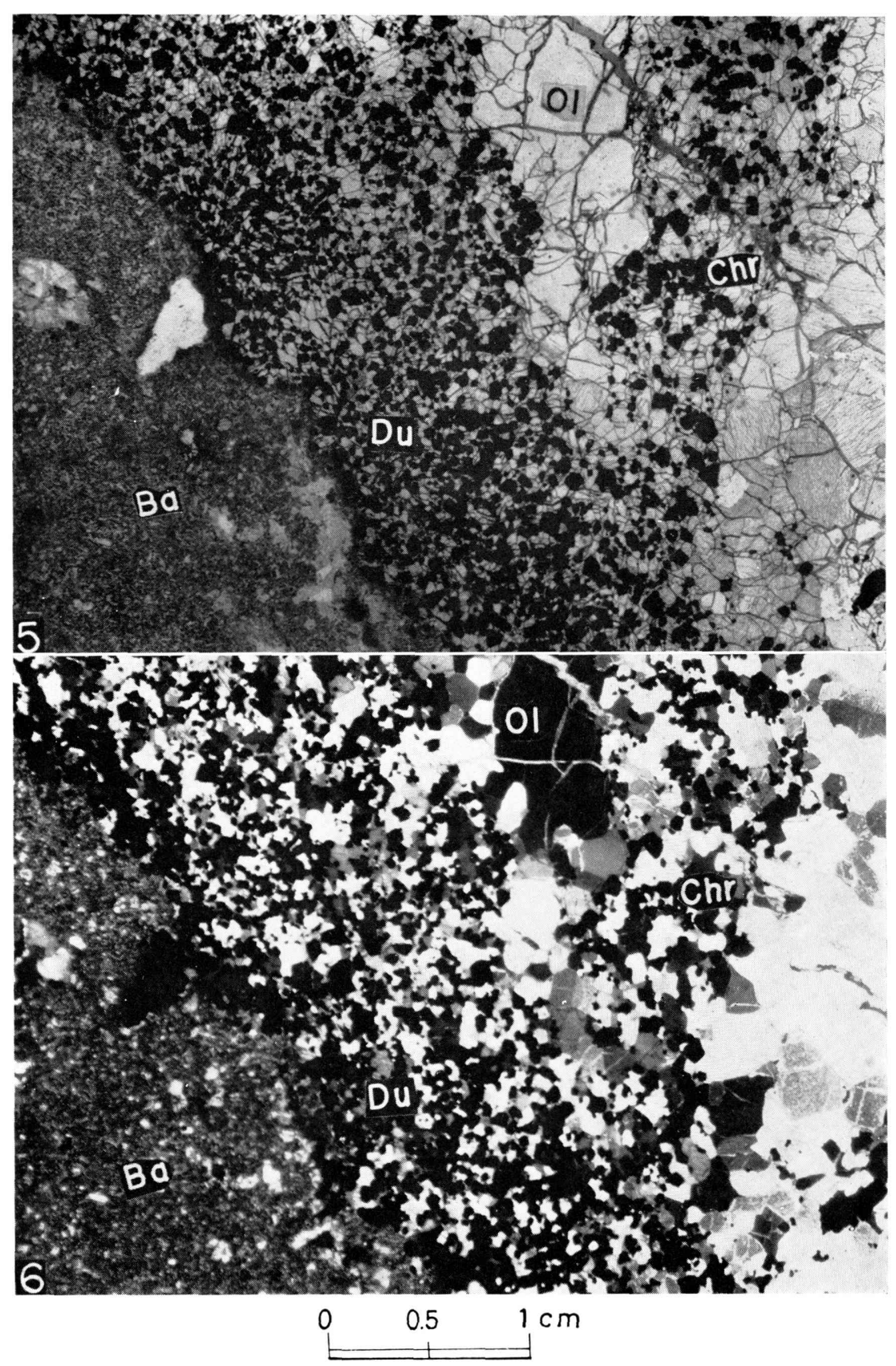

K. Ishibashi: Basic and Ultrabasic Inclusions 
Plate 21 


\section{Explanation of Plate 21}

Fig. 7. Photomicrograph of the contact between olivine-free gabbro (Ga) and basalt $(\mathrm{Ba})$. The large phenocryst-like minerals $(\mathrm{Au}, \mathrm{Hy})$ in the basalt seem to have come from gabbro inclusions. The boundary line of the gabbro $\mathrm{Ga}$ and basalt $\mathrm{(Ba}$ ) show a very irregular line, open nicols.

Fig. 8. Photomicrograph of a veinlet of aluminous augite and aluminous hypersthene ( $\mathrm{Al}-\mathrm{Px})$ cutting through a dunite inclusions $(\mathrm{Du})$, open nicols. 


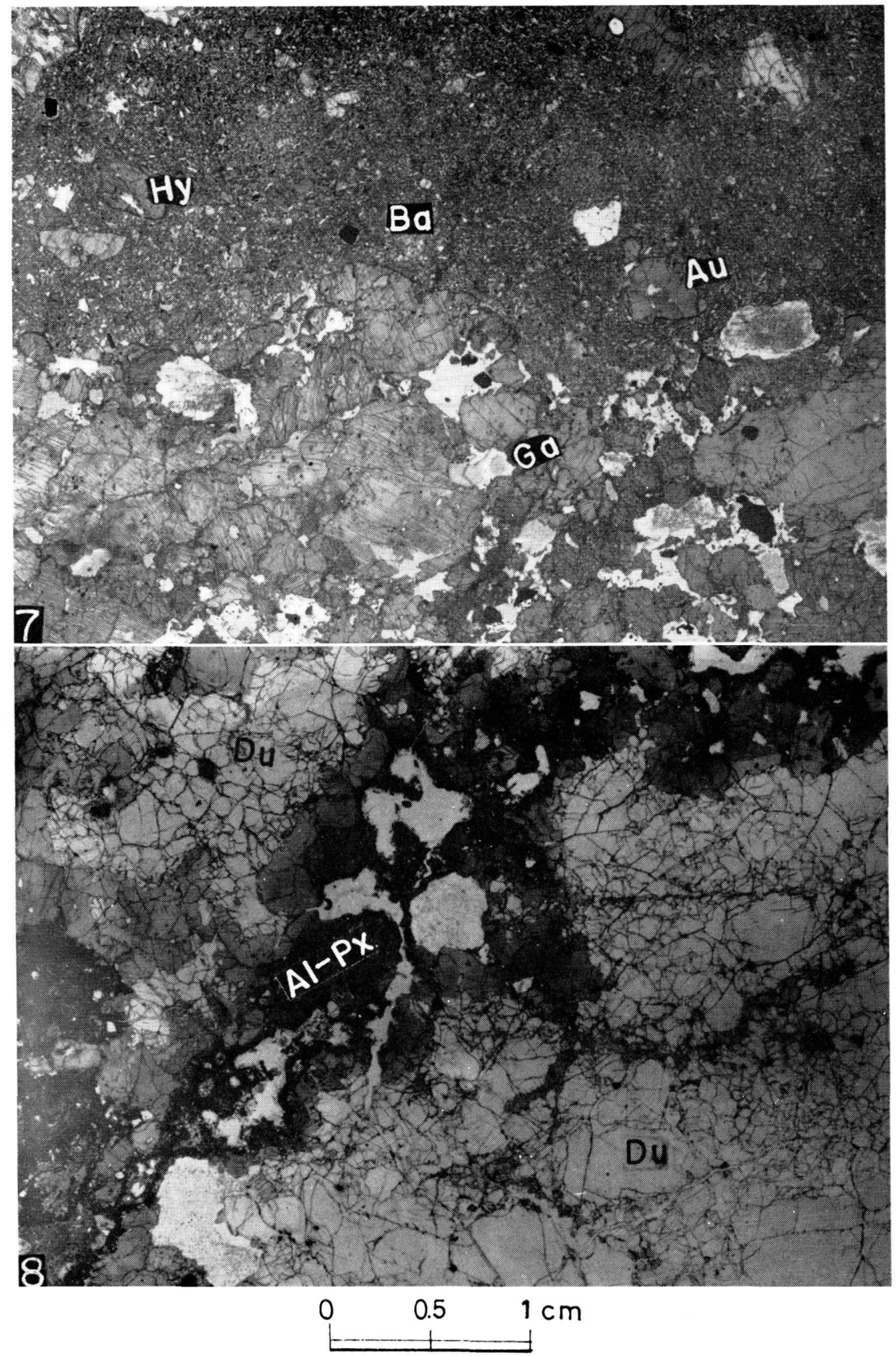

K. IsHIBASHI: Basic and Ultrabasic Inclusions 
Plate 22 


\section{Explanation of Plate 22}

Fig. 9. Photomicrograph of a veinlet of basalt (Ba) cutting through a dunite $(\mathrm{Du})$ inclusion. The outer part of the inclusions $(\mathrm{Du})$ is surrounded by the aliminous augite and hypersthene (Al-Px), open nicols.

Fig. 10. Crossed nicols of Fig. 9 .

Fig. 11. Photomicrograph of a large phenocryst-like olivine (Ol) which seems to have been derived from dunite or peridotite inclusions. It has striation and irregular parting, open nicols.

Fig. 12. Crystal of olivine $(\mathrm{Ol})$ in the dunite inclusion showing translation lammelae, crossed nicols. 

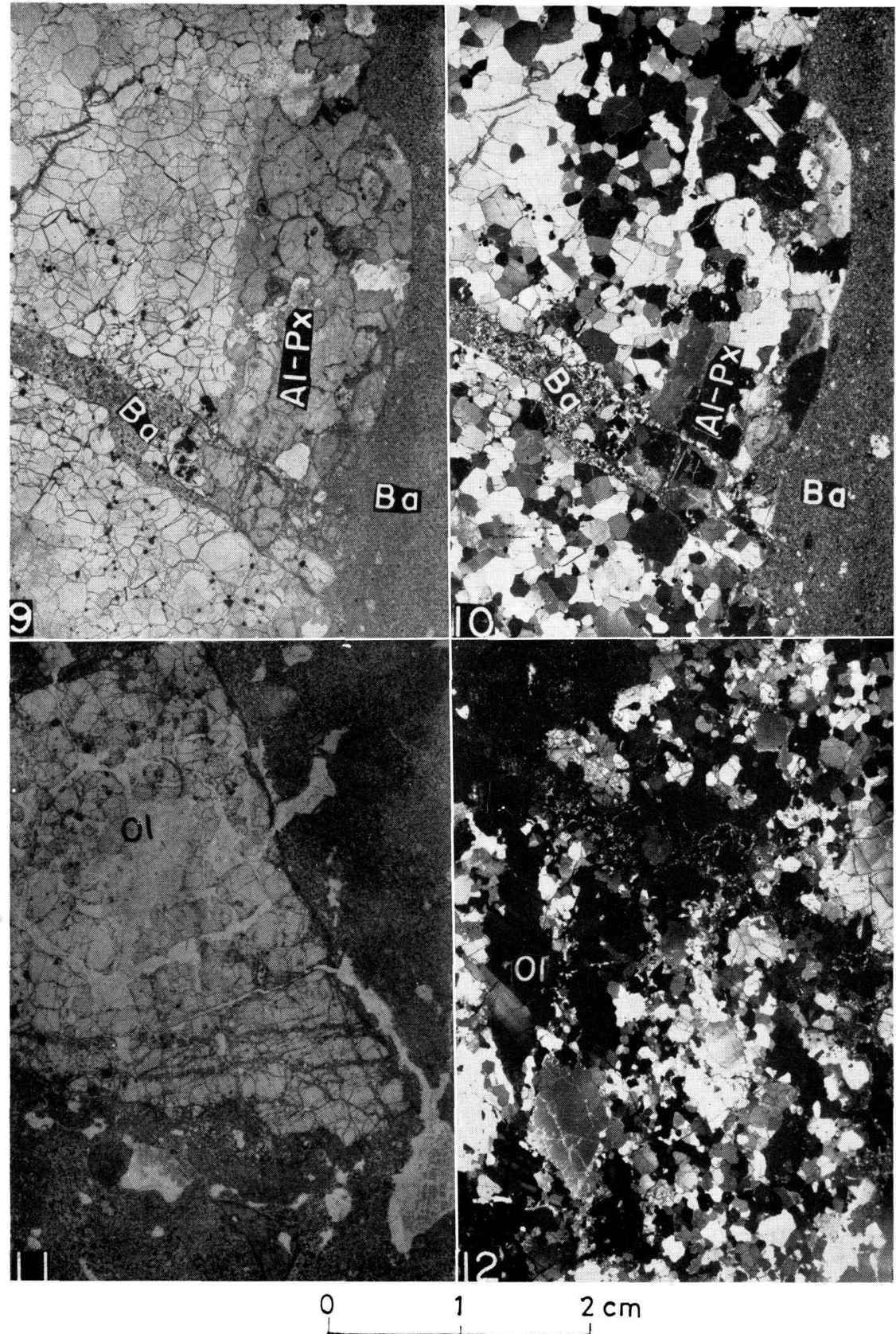

K. IshiBASHI: Basic and Ultrabasic Inclusions 
Plate 23 


\section{Explanation of Plate 23}

Fig. 13. A small inclusion of gabbro (Ga) showing irregular outline in basaltic host rock $(\mathrm{Ba})$, the outer margin changed to small aggregate of olivine, augite, plagioclase and glass. Aluminous hypersthene (Hy) changed it margin to small aggregate of olivine and/or pigeonite and plagioclase. Disintegrated crystal of olivine, augite, plagioclase and opaque minerals are scattered as phenocryst-like minerals in basalt, open nicols.

Fig. 14. Crossed nicols of Fig. 13.

Fig. 15. Photomicrograph showing a boundary between a peridotite inclusion $(\mathrm{Pe})$ and basalt $(\mathrm{Ba})$. The outer part of the peridotite is surrounded by aluminous pyroxenite ( $\mathrm{Al}-\mathrm{Px})$. The large phenocryst-like mineral $(\mathrm{Au})$ which seems to have been derived from basic inclusions is almost vitrified, open nicols.

Fig. 16. Crossed nicols of Fig. 15. 


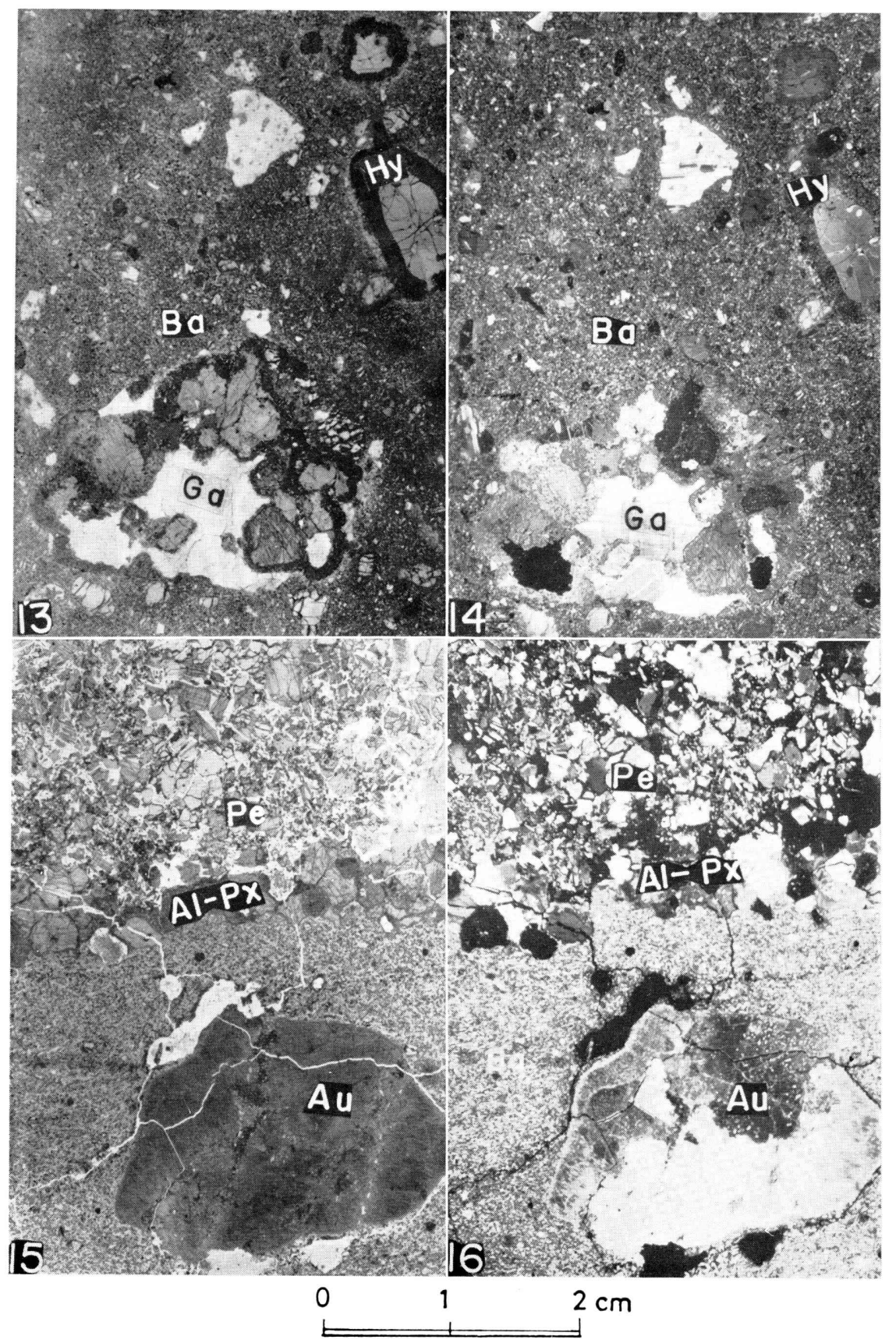

K. IshiBAshi: Basic and Ultrabasic Inclusions 
Plate 24 


\section{Explanation of Plate 24}

Fig. 17. Photomicrograph showing the boundary between Cr-diopside peridotite $(\mathrm{Pe})$ and basalt $(\mathrm{Ba})$. The former is covered by aluminous pyroxenite (Al-P'x) as a reaction rim around it. The opaque minerals in aluminous pyroxenite is aluminous spinel ( $\mathrm{Sp})$, open nicols.

Fig. 18. Crossed nicols of Fig. 17.

Fig. 19. Photomicrograph of a gabbro inclusion $(\mathrm{Ga})$ and sporadically scattered large phenocrysts of aluminous hypersthene $(\mathrm{Hy})$ and aluminous augite $\mathrm{Au}$ which are decomposed to fine grained olivine, plagioclase, opaque mineral and augite at the margin, open nicols.

Fig. 20. Crossed nicols of Fig. 19. 


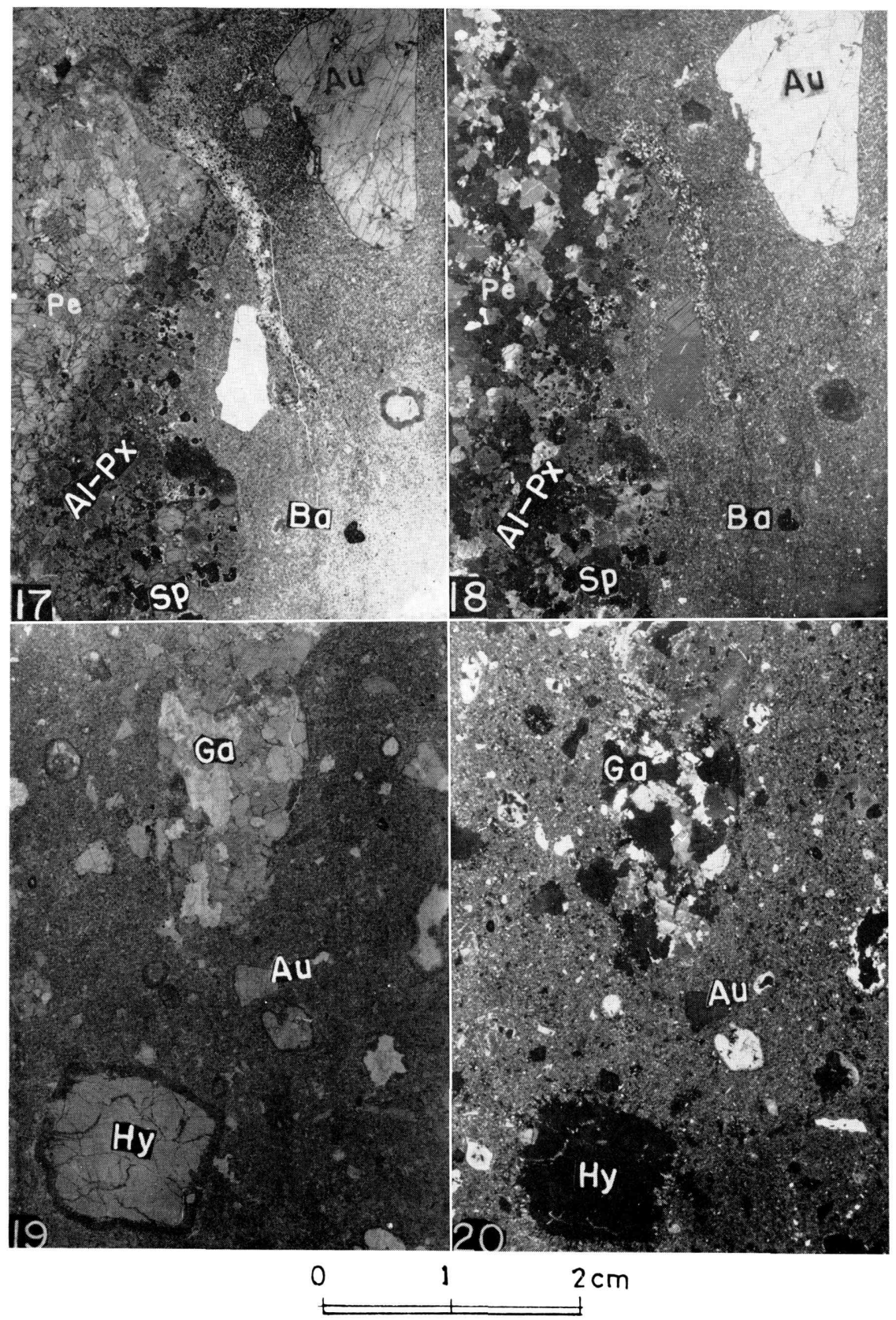

K. Ishibashi : Basic and Ultrabasic Inclusions 
Plate 25 


\section{Explanation of Plate 25}

Fig. 21. Photomicrograph showing a boundary between a gabbro inclusion (Ga) and basalt (Ga). The opaque minerals in the gabbro are aluminous spinel (Sp), Crossed nicols.

Fig. 22. Photomicrogrpah of gabbro inclusion consisting of aluminous augite $(\mathrm{Au})$, aluminous hypersthene $(\mathrm{Hy})$, plagioclase $(\mathrm{Pl})$ and opaque minerals $(\mathrm{Op})$. The aliminous augite and aluminous hypersthene show exsolution texture, crossed nicols.

Fig. 23. Photomicrograph showing a boundary of basalt (Ba) and $\mathrm{Cr}-$ pyroxenite $(\mathrm{Cr}-\mathrm{Px})$ which consists of chromian diopside (Di) of unequal size. The large crystal of chromian diopside (Di) have numerous inclusions of opaque minerals, whereas the small crystals of chrominian diopside have no opaque minerals and very transparent, crossed nicols.

Fig. 24. Photomicrograph showing a gabbro in basalt $(\mathrm{Ba})$. The outer margin of the phenocryst minerals $(\mathrm{Au})$ are corroded by the basaltic magma, forming rounded shape, crossed nicols. 


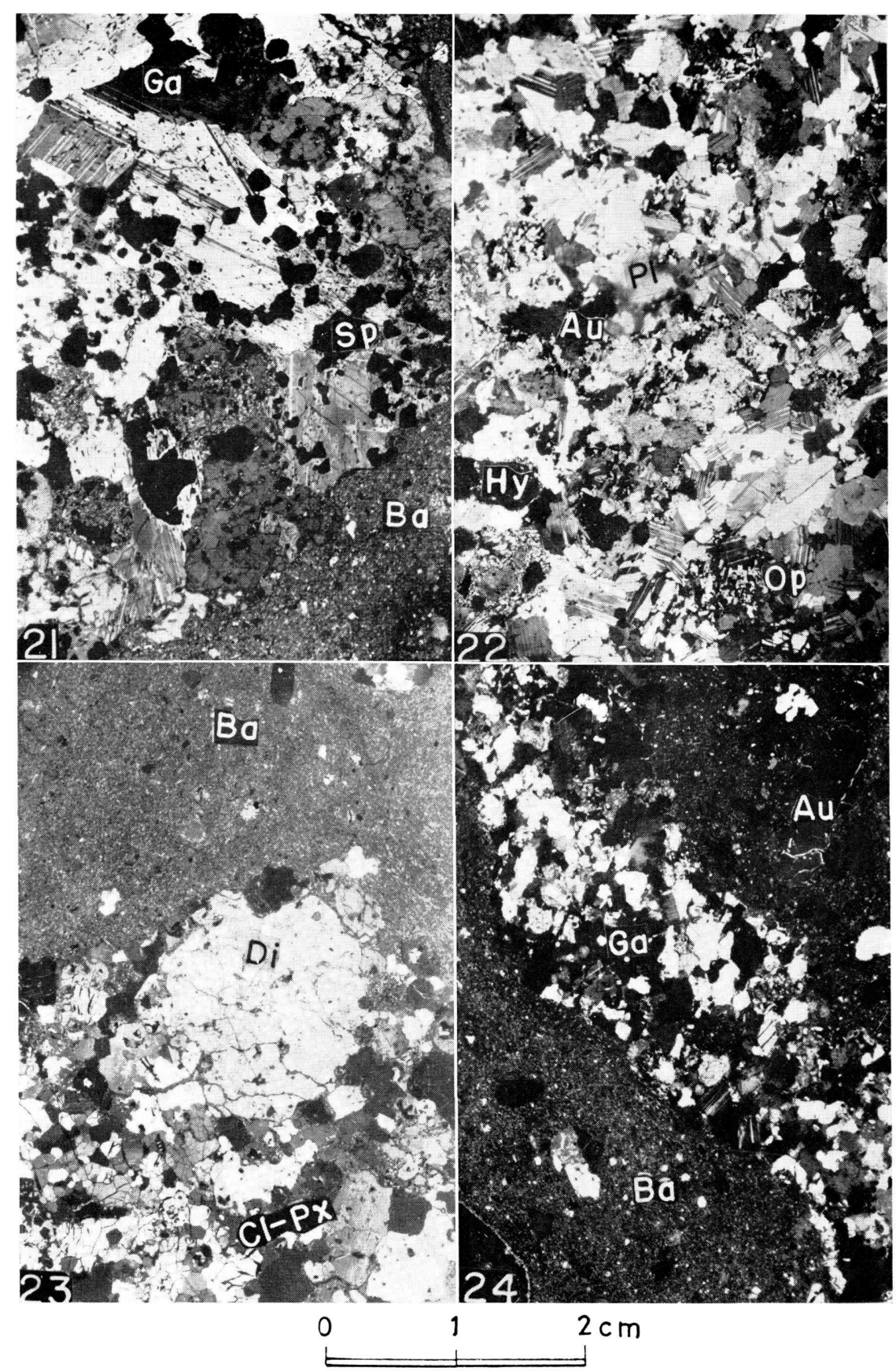

K. Ishibashi: Basic and Ultrabasic Inclusions 